Aus der Klinik für Gastroenterologie und gastrointestinale Onkologie

(Prof. Dr. med. V. Ellenrieder)

der Medizinischen Fakultät der Universität Göttingen

\title{
Frakturrisikoevaluation mithilfe
}

\author{
verschiedener nationaler und
}

\section{internationaler Risikoscores bei}

\section{Frauen}

\author{
INAUGURAL-DISSERTATION \\ zur Erlangung des Doktorgrades \\ der Medizinischen Fakultät der \\ Georg-August-Universität zu Göttingen
}

vorgelegt von

Anna Giessel

aus

Oldenburg (in Oldenburg)

Göttingen 2020 
Dekan:

Referent/in:

Ko-Referent/in:

Drittreferent/in:
Prof. Dr. med. W. Brück

Prof. Dr. med. H. Siggelkow

PD Dr. Daniel Hoffmann

Prof. Dr. Margarete Schön

Datum der mündlichen Prüfung: 17.11.2021 
Hiermit erkläre ich, die Dissertation mit dem Titel „Frakturrisikoevaluation mithilfe verschiedener nationaler und internationaler Risikoscores bei Frauen" eigenständig angefertigt und keine anderen als die von mir angegebenen Quellen und Hilfsmittel verwendet zu haben.

Göttingen, den 


\section{Inhaltsverzeichnis}

Abbildungsverzeichnis .......................................................................................II

Tabellenverzeichnis ........................................................................................... V

Abkürzungsverzeichnis ............................................................................. VII

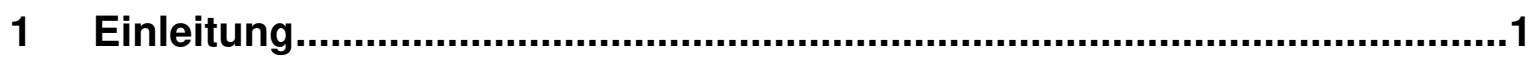

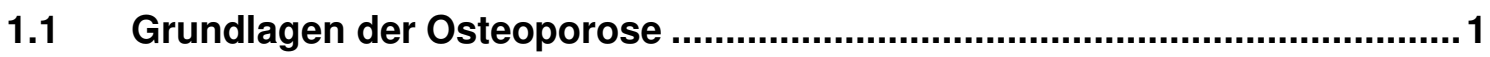

1.2 Scores zur Frakturrisikoevaluation ........................................................... 12

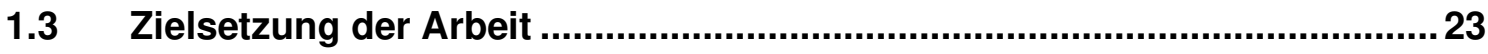

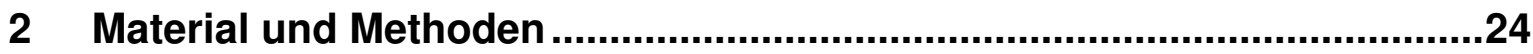

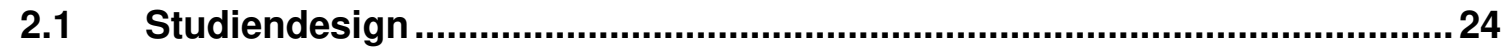

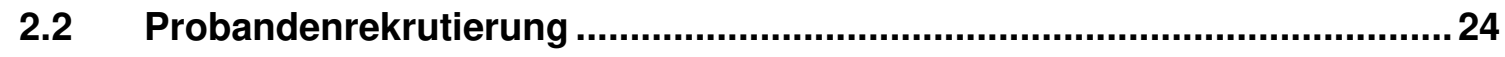

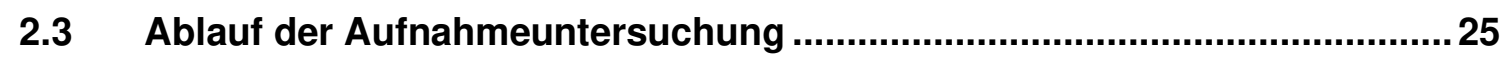

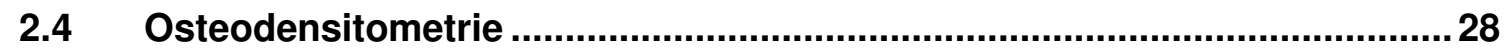

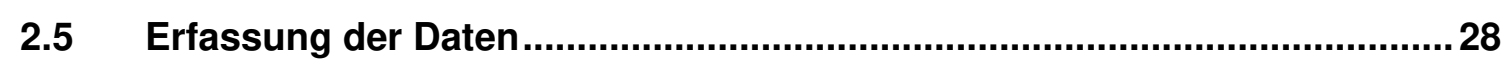

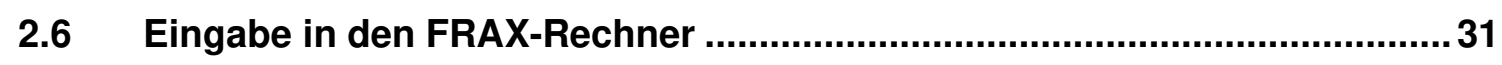

2.7 Risikoevaluation nach der DVO-Leitlinie von 2009 .......................................34

2.8 Risikoermittlung mittels nichtpubliziertem Risikomodell (DVO+) ................36

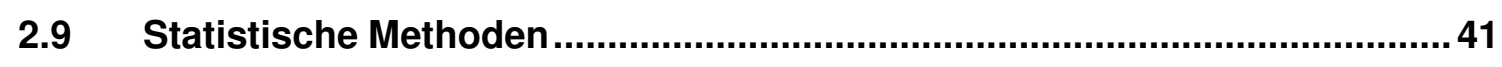

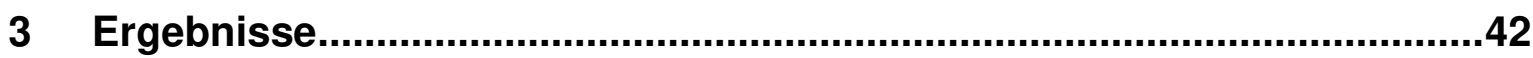

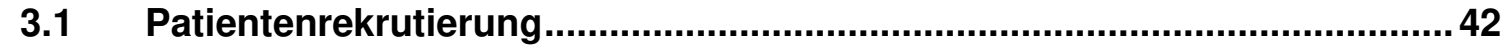

3.2 Patientencharakterisierung .................................................................. 42

3.3 10-Jahres-Frakturwahrscheinlichkeiten nach DVO-Leitlinien ..................... 45

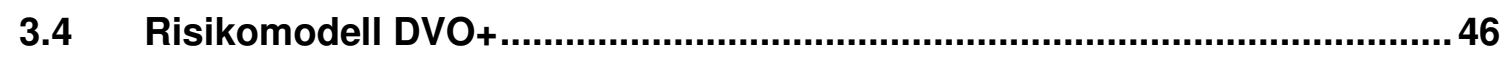

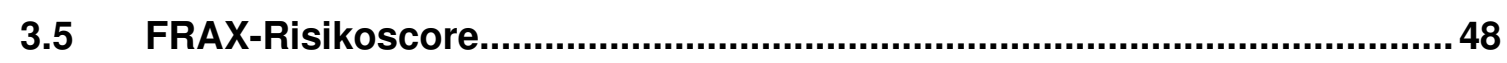

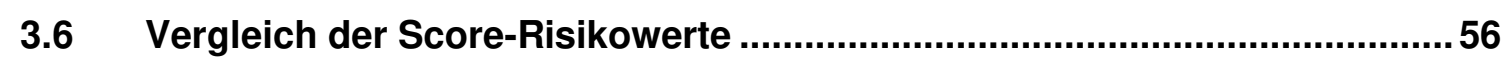

3.7 Vergleich der Therapieindikationen der Scores .........................................61

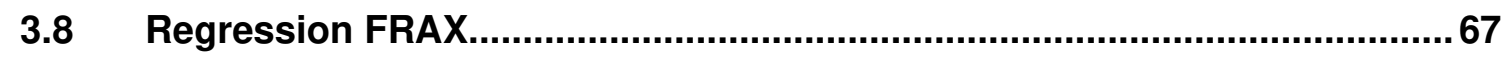

3.9 Vergleich FRAX und modifizierter FRAX „endo“..........................................69

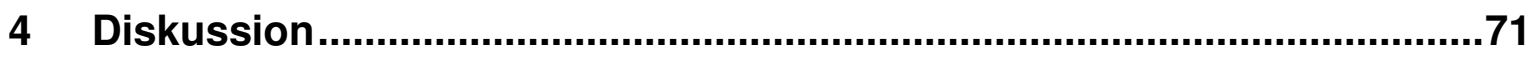

4.1 Basisdaten innerhalb des Frauenkollektivs...............................................71

4.2 Score-Vergleicah............................................................................... 74

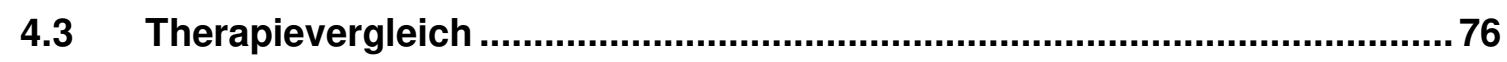

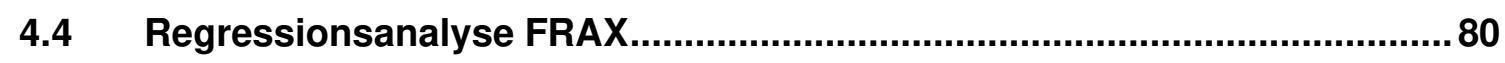

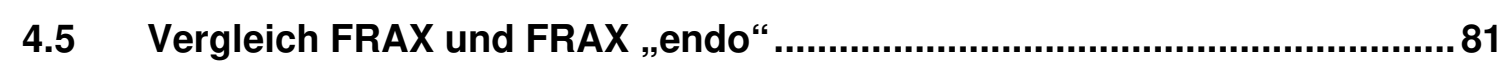




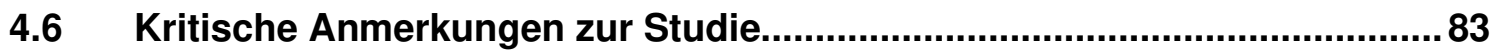

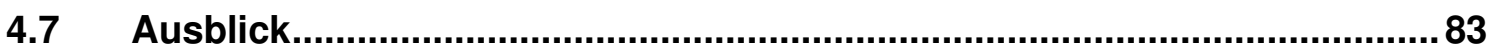

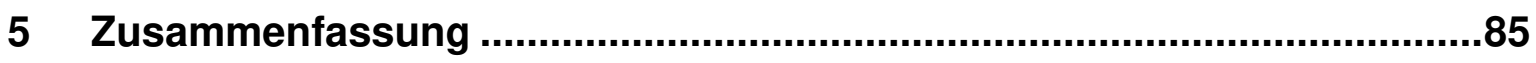

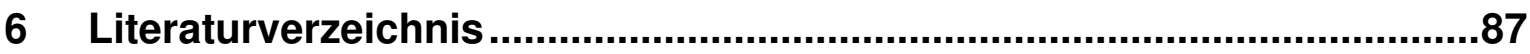




\section{Abbildungsverzeichnis}

Abbildung 1: Indikation für eine spezifische medikamentöse Therapie.........16

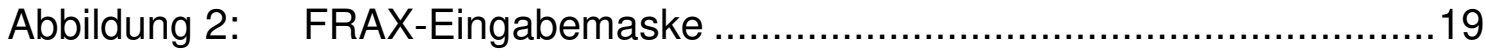

Abbildung 3: $\quad$ Q-Fracture-Eingabemaske............................................20

Abbildung 4: Garvan-Eingabemaske .................................................22

Abbildung 5: Gynäkologische Anamnese .........................................26

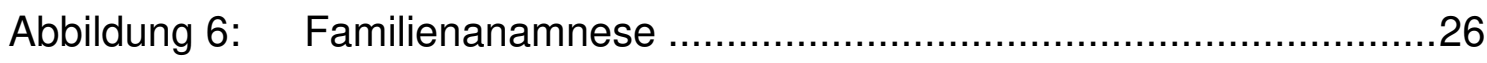

Abbildung 7: Medikamentenanamnese...........................................26

Abbildung 8: Erfassung von Ernährungs- und Lebensgewohnheiten, sowie Registrierung etwaiger Größenabnahme ...........................27

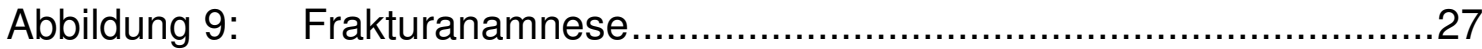

Abbildung 10: Indikation für eine spezifische Diagnostik der Osteoporose.....35

Abbildung 11: Indikationen für eine spezifische medikamentöse Therapie.....36

Abbildung 12: Neues Risikoevaluationsmodell ....................................40

Abbildung 13: Risikogruppeneinteilung nach DVO-Leitlinien.......................46

Abbildung 14: FRAX-Mittelwerte aufgetragen gegen Altersgruppen .............49

Abbildung 15: Häufigkeitsverteilung „Vorausgegangene Fraktur ja/nein“ .......50

Abbildung 16: Verschiedene Frakturtypen..............................................51

Abbildung 17: Häufigkeitsverteilung „Hüftfraktur eines Elternteils ja/nein“......51

Abbildung 18: Häufigkeitsverteilung „Gegenwärtiges Rauchen ja/nein“ .........52

Abbildung 19: Häufigkeitsverteilung „Glukokortikoide ja/nein“....................53

Abbildung 20: Häufigkeitsverteilung „Rheumatoide Arthritis ja/nein“ .............53

Abbildung 21: Häufigkeitsverteilung „sekundäre Osteoporose ja/nein“..........54

Abbildung 22: Häufigkeitsverteilung primärer und sekundärer Osteoporosepatientinnen in DVO-Risikogruppen ...................54

Abbildung 23: Häufigkeitsverteilung „Alkoholkonsum ja/nein“ .......................55

Abbildung 24: Vergleich der 1-Jahres-Frakturrisikowerte von FRAX und

DVO+

Abbildung 25: Therapiegrenzen im FRAX anteilig an Patientinnen mit DVO 10-Jahres-Frakturrisiko > 30 \%.......................................63

Abbildung 26: Therapiegrenzen im FRAX anteilig an Patientinnen mit DVO 10-Jahres-Frakturrisiko 20-30 \%........................................65

Abbildung 27: Therapieempfehlung.................................................66 
Abbildung 28: Patientinnenbezogene Übereinstimmung der

Therapieempfehlungen.......

67 


\section{Tabellenverzeichnis}

Tabelle 1: Indikation zur Basisdiagnostik anhand der DVO-Leitlinie

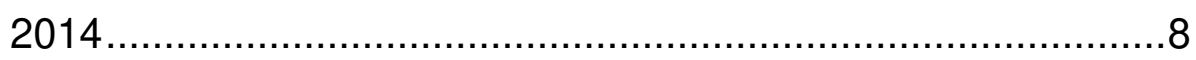

Tabelle 2: $\quad$ Einfließende Risikofaktoren für Risikomodell DVO+ ................38

Tabelle 3: $\quad$ Basisdaten der Patientinnen ................................................43

Tabelle 4: Häufigkeitsverteilung in Altersgruppen nach

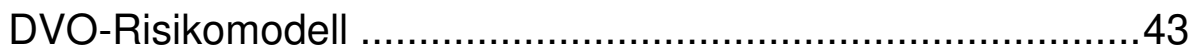

Tabelle 5: $\quad$ Basisdaten zu Größe, Gewicht und BMI ...............................44

Tabelle 6: Knochendichtemessungsergebnisse an drei verschiedenen Lokalisationen (Wirbelsäule, Femur gesamt und Femurhals) ..45

Tabelle 7: Häufigkeitsverteilung der relativen Risikowerte im DVO+........47

Tabelle 8: $\quad$ 1-Jahres-Frakturrisiko in DVO+ .......................................48

Tabelle 9: $\quad$ FRAX-10-Jahres-Risikowerte aller Patientinnen.....................48

Tabelle 10: FRAX-Mittelwerte innerhalb Altersgruppen nach Risikomodell

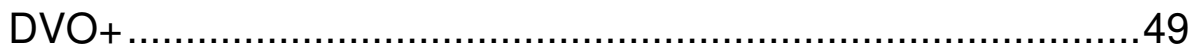

Tabelle 11: Knochendichtemessung am Femurhals................................55

Tabelle 12: Vergleich der durchschnittlichen T-Scores am Femurhals mit und ohne vorausgegangene Fraktur ......................................56

Tabelle 13: $\quad$ Vergleich der durchschnittlichen T-Scores Femurhals

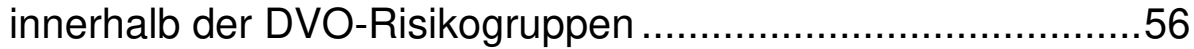

Tabelle 14: DVO- und FRAX-Vergleich ohne Knochendichtemessung ......57

Tabelle 15: Übereinstimmung der 10-Jahres-Frakturwahrscheinlichkeiten bei Gruppierung der FRAX-Werte ohne BMD .........................57

Tabelle 16: DVO- und FRAX-Vergleich bei eingeschlossener Knochen-

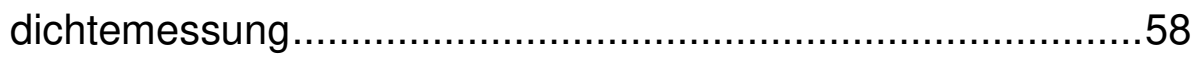

Tabelle 17: Übereinstimmung der 10-Jahres-Frakturwahrscheinlichkeiten bei Gruppierung der FRAX-Werte mit BMD ...........................58

Tabelle 18: Übereinstimmung der 10-Jahres-Frakturwahrscheinlichkeiten bei Gruppierung der DVO+-Werte hochgerechnet auf 10 Jahre .59

Tabelle 19: Korrelation der gruppierten Scores DVO, FRAX MOF ohne und mit BMD und DVO+ 
Tabelle 20: Therapieindikation laut FRAX mit publizierter internationaler $\geq 20 \%$ MOF und $\geq 3 \%$ HF 10-Jahres-FrakturrisikoTherapie-grenze

Tabelle 21: Therapieindikation laut FRAX mit publizierter $\geq 14 \%$ MOF und $\geq 3 \%$ HF 10-Jahres-Frakturrisiko-Therapiegrenze..... .62

Tabelle 22: $\quad$ FRAX-Mittelwerte bezogen auf Patientinnen mit DVO 10Jahres-Frakturrisiko > $30 \%$ 62

Tabelle 23: FRAX-Mittelwerte bezogen auf Patientinnen mit DVO 10Jahres-Frakturrisiko 20-30\% 64

Tabelle 24: Regression FRAX MOF mit und ohne BMD............................68

Tabelle 25: Regression FRAX HF mit und ohne BMD ..............................69

Tabelle 26: Vergleich FRAX und FRAX "endo" .......................................70

Tabelle 27: Vergleich der Basisdaten mit anderen Studien ........................72

Tabelle 28: Gegenüberstellung DVO und FRAX ……............................74 


\section{Abkürzungsverzeichnis}

\begin{tabular}{|c|c|}
\hline AP & Alkalische Phosphatase \\
\hline BEST & Bone Evaluation Study \\
\hline BMD & Bone mineral density \\
\hline BMI & Body Mass Index \\
\hline COPD & Chronisch obstruktive Lungenerkrankung \\
\hline CRP & C-reaktives Protein \\
\hline DPD & Desoxypyridinolin \\
\hline DVO & Dachverband Osteologie \\
\hline DXA & Dual-X-Ray-Absorptiometrie \\
\hline HF & Hip Fracture \\
\hline V-GT & ү-Glutamyltransferasen \\
\hline MBDP & Manitoba Bone Density Program \\
\hline MGUS & Monoklonale Gammopathie unklarer Signifikanz \\
\hline MOF & Major Osteoporotic Fracture \\
\hline NOF & National Osteoporosis Foundation \\
\hline OPO & Osteoporose \\
\hline PPI & Protonenpumpeninhibitor \\
\hline QCT & Quantitative Computertomographie \\
\hline RANKL & Receptor Activator of NF-кB Ligand \\
\hline $\mathrm{TSH}$ & Thyroidea-stimulierendes Hormon \\
\hline WHO & World Health Organization \\
\hline
\end{tabular}




\section{Einleitung}

\subsection{Grundlagen der Osteoporose}

\subsubsection{Osteoporose-Definition}

Die Osteoporose ist definiert als eine systemische Skeletterkrankung, charakterisiert durch eine niedrige Knochenmasse und eine mikroarchitektonische Verschlechterung des Knochengewebes. Es tritt ein konsekutiver Anstieg der Knochenfragilität mit Neigung zu Frakturen auf. Bei der manifesten Osteoporose sind bereits eine oder mehrere Frakturen als Folge der Osteoporose aufgetreten. Als typisch gelten Schenkelhals-, Wirbelkörper- und handgelenksnahe Frakturen (Consensus Development Conference 1993).

Während die klinische Relevanz der Osteoporose vor allem im Auftreten von Knochenbrüchen und deren Folgen begründet liegt, ist der für die klinische Diagnostik erhebliche Faktor die erniedrigte Knochendichte (Bone mineral density, BMD).

Die WHO definierte im Jahre 1994 eine Osteoporose als vorliegend, sobald der Knochenmineralgehalt in einer Knochendichtemessung mittels Dual-X-RayAbsorptiometrie gemessen an Lendenwirbelsäule und/oder am proximalen Femur um $\geq 2,5$ Standardabweichungen unter dem Mittelwert einer 20- bis 29-jährigen Frau abweicht (T-Score) (Kanis et al. 1994). Einzuschränken ist jedoch bei dieser operationalen Definition, dass sie erst nach Ausschluss anderer, den Knochenmineralgehalt beeinflussenden Erkrankungen angewendet werden darf. Seit 1994 hat sich die Vorgehensweise deutlich verändert, da neben der Knochendichte multiple andere Faktoren das Frakturrisiko beeinflussen, allen voran das Alter und das Geschlecht (Cruz et al. 2018). Die Diagnosestellung anhand des T-Scores der DXA-Messung sollte demnach nicht nur aus Betrachtung der Knochendichtemessungsergebnisse bestehen, sondern muss aus dem Kontext der Vorgeschichte der jeweiligen Patientin heraus erfolgen (DVO 2014).

\subsubsection{Epidemiologie}

Im Jahr 2009 litten rund 6,3 Millionen Menschen in Deutschland an einer Osteoporose. Davon waren 5,2 Millionen Patienten Frauen und 1,1 Millionen Männer. Die in der Bone Evaluation Study (BEST) mit Daten der Techniker Krankenkasse ermittelte Osteoporose-Prävalenz bei über 50-Jährigen lag demnach bei $14 \%$. Der 
höhere prozentuale Anteil fiel dabei den Frauen mit $24 \%$ zu, die OsteoporosePrävalenz bei den Männern lag niedriger, bei rund $6 \%$. Altersassoziiert steigt laut dieser Daten die Prävalenz von $12 \%$ im Alter von 50 Jahren bis auf $48 \%$ bei den Frauen, die das 75. Lebensjahr erreicht haben. Pro Jahr treten 885.000 Neuerkrankungen auf. Jede vierte Frau und jeder 17. Mann mit einem Alter über 50 Jahre ist betroffen (Hadji et al. 2013a).

In Europa wird die Zahl der an Osteoporose erkrankten Menschen auf 22 Millionen Frauen und 5,5 Millionen Männer geschätzt. Rund 3,5 Millionen neue osteoporotische Frakturen wurden registriert, darunter 610.000 Hüft- und 520.000 Wirbelkörperfrakturen (Kanis et al. 2016b). Weltweit sind nach Angaben der „World Health Organization“ (WHO) aus dem Jahre $199430 \%$ aller postmenopausalen Frauen von osteoporotischen Knochenveränderungen betroffen (Häussler et al. 2006; Strom et al. 2011; Lorentzon und Cummings 2015). Das Lebenszeitrisiko für eine Handgelenks-, Hüft- oder Wirbelkörperfraktur wird in entwickelten Ländern auf $30 \%$ bis $40 \%$ geschätzt und liegt damit nahe dem Risiko für die koronare Herzerkrankung. Weltweit kann die Osteoporose für 8,9 Millionen Frakturen jährlich verantwortlich gemacht werden (Kanis 2007).

Mit steigender Lebenserwartung innerhalb der Bevölkerung wird auch die Frakturrate zunehmen. Während jede achte Frau an Brustkrebs erkrankt, erleidet sogar jede dritte Frau einen Knochenbruch, der sich durch das Vorhandensein einer Osteoporose erklären lässt. Erreicht eine Frau das 50. Lebensjahr, liegt das geschätzte Risiko für das Auftreten einer Wirbelkörperfraktur bei $32 \%$. Das Risiko für das Auftreten von einer Unterarmfraktur liegt demnach bei $16 \%$ und das einer Oberschenkelhalsfraktur nur eben darunter bei $15 \%$. Die dadurch verursachten Kosten für das Gesundheitssystem belaufen sich in der Bundesrepublik Deutschland auf rund fünf Milliarden Euro. Bei konsequent durchgeführter Therapie nach Leitlinie könnten vermutlich rund die Hälfte der Frakturen verhindert und die entstehenden Kosten somit erheblich gesenkt werden (Bartl 2011).

Zahlreiche Studien ergeben weiterhin, dass vorausgegangene Frakturen als Prädiktor für das Auftreten zukünftiger Frakturen fungieren. Tritt beispielsweise eine vertebrale Fraktur auf, besteht für eine postmenopausale Frau eine zwanzigprozentige Chance im Folgejahr eine weitere Wirbelkörperfraktur zu erleiden (Lindsay et al. 2001). Das Risiko einer Hüftfraktur bei stattgehabter Handgelenksfraktur erhöht sich bei postmenopausalen Frauen um über $50 \%$. Nach Fraktur eines Wir- 
belkörpers ist das Risiko eine Hüftfraktur zu erleiden bei diesen Frauen mehr als doppelt so hoch (Haentjens et al. 2003).

Die folgenschwerste Fraktur bezogen auf Morbidität, Mortalität und Kostenfaktor ist die Hüftfraktur. Die wirtschaftlichen Kosten osteoporotischer Frakturen werden auf 37 Milliarden Euro geschätzt - dabei nehmen inzidente Frakturen zwei Drittel der Kosten ein, Langzeitfrakturnachsorge nimmt einen Anteil von $29 \%$ und die pharmakologische Prävention nur etwa $5 \%$ der Kosten ein. Es wird erwartet, dass diese Kosten bis zum Jahr 2025 nochmals um $25 \%$ ansteigen (Hernlund et al. 2013).

\subsubsection{Osteoporose-Einteilung}

Die Osteoporose lässt sich nach verschiedensten Kriterien unterteilen. Die vielleicht einfachste Unterteilung ist die zwischen generalisierten Osteoporosen, die das ganze oder zumindest einen großen Anteil des knöchernen Skeletts betreffen, von den lokalisierten Formen der Osteoporose, die auf einen Teil des Skeletts beschränkt sind. Zur letztgenannten Form wird zum Beispiel die Inaktivitätsosteoporose einer Extremität nach Immobilisation, wie durch eine Schienung nach aufgetretener Fraktur, gezählt. Die häufigere generalisierte Osteoporose betrifft, anders als der Name vermuten lässt, selten das gesamte Skelett, tritt aber meist symmetrisch, bevorzugt am Achsenskelett auf.

Betrachtet man Alter und Geschlecht der Patientinnen mit Osteoporose, ergibt sich eine erste Einteilungsform. Die häufigste Form der Osteoporose nach dieser Einteilung ist die postmenopausale Osteoporose, auch Osteoporose Typ I genannt. Sie tritt vor allem bei Frauen zwischen dem 51. und 73. Lebensjahr auf und ist durch den Ausfall der Funktion der Ovarien bedingt. Eine vergleichbare Pathologie durch einen Mangel an Testosteron wurde allerdings mittlerweile auch bei Männern registriert. Das Geschlechterverhältnis beträgt etwa 20:1.

Durch den Mangel an Östrogen entsteht über die vermehrte Rekrutierung von Osteoklasten eine erhöhte Calcium-Mobilisation aus dem Knochen. Regulativ sinkt die Parathormonsekretion in den Nebenschilddrüsen, die Niere bildet weniger Calcitriol, die aktive Form des Vitamin D, und die Resorption von Calcium aus Niere und Darm sinkt. Auch der von Calcitriol geförderte Prozess des Einbaus von calciumhaltigem Hydroxylapatit in die Knochenmatrix wird somit gedämpft. Allein die Empfindlichkeit des Knochens für die resorptiven Effekte des Parathormons steigt. Dies führt zu einer negativen Bilanz des Knochenstoffwechsels. Es folgt ein Abbau 
des spongiösen Knochenanteils, jedoch auch eine Zunahme der kortikalen Porosität und damit Abnahme der Kortikalissubstanz vor allem im Bereich von Wirbelkörper- und Oberschenkelknochen. Eine erhöhte Frakturneigung resultiert (Ahmed et al. 2015; Tella und Gallagher 2014).

Die senile Osteoporose entspricht einer Typ-II-Osteoporose und tritt vor allem bei Patientinnen nach dem 70. Lebensjahr auf. Die Pathogenese ist vielschichtig. Hier liegt der Verlust der Knochenmasse weniger in einer erhöhten Aktivität der Osteoklasten sondern viel mehr in einer verminderten Anzahl an Osteoblasten begründet.

Ein defekter Vitamin-D-Metabolismus, sowie eine verminderte Calciumresorption aus dem Darm führen zusätzlich oft reaktiv zu einem sekundären Hyperparathyreoidismus mit gesteigerter Parathormonsekretion, sodass zusätzlich ebenfalls eine Stimulation der Calciummobilisation aus dem Knochen und somit eine Stimulation der Aktivität der Osteoklasten besteht. Hinzu kommen altersbedingte Faktoren wie Immobilität und ein bereits atrophisches Knochengewebe, die die Knochensituation weiterhin verschlechtern. Im Vergleich zur Typ-I-Osteoporose ist bei der senilen Osteoporose auch der kortikale Anteil des Knochens häufig betroffen. Nahezu 80 \% aller osteoporosebedingten Frakturen können diesem Typ zugeordnet werden. Das Verhältnis Frauen zu Männern beträgt bei dieser Form nur noch 2:1 (Bartl 2011).

Wesentlich seltener tritt die Osteoporose in jüngeren Jahren auf. Man unterscheidet die idiopathische juvenile Osteoporose die vor allem bei Kindern zwischen dem achten und dem vierzehnten Lebensjahr auftritt, und sich durch starke Rückenschmerzen bei Wirbelkörperkompressionsfrakturen äußert, und die idiopathische Osteoporose junger Erwachsener. Letztere befällt vor allem Männer ab dem 30. Lebensjahr. Auch hierbei kommt es vor allem zu Wirbelkörperfrakturen. Der differentialdiagnostischen Abklärung kommt vor allem bei dem Verdacht auf Osteoporose in jungem Alter eine erhebliche Bedeutung zu. Vor allem das Vorliegen einer Osteogenesis imperfecta sollte bei gehäuftem Auftreten von Frakturen ausgeschlossen werden (Saraff und Hogler 2015).

Eine weitere Einteilungsform ergibt sich unter Berücksichtigung des Knochenumsatzes. Niedriger Knochenumsatz (low turnover) und hoher Knochenumsatz (high turnover) werden unterschieden. Bei der Low-Turnover-Form der Osteoporose liegt pathogenetisch eine verminderte Knochenneubildung zugrunde, während bei 
der High-Turnover-Form der Osteoporose eine exzessive Steigerung der Knochenresorption mit reaktiv auch leicht gesteigerter Knochenneuformation vorhanden sind. Des Weiteren gibt es die Osteoporosen mit besonders raschem Knochenverlust genannt Very-High-Turnover- oder Fast-Loosers-Osteoporose.

Hinsichtlich der Ätiologie unterscheidet man die primären von den sekundären Formen der Osteoporose. Zu den primären oder auch idiopathischen Osteoporosen werden vor allem die senilen und postmenopausalen Osteoporosen gezählt. Sekundäre Osteoporosen sind die Formen der Osteoporose, denen eine Grundkrankheit zugeordnet werden kann. Obwohl sie nur fünf Prozent aller Osteoporosen umfassen, verursachen sie jedoch $20 \%$ aller osteoporosebedingten Frakturen. Ursprünge einer sekundären Osteoporose lassen sich unter anderem in gastroenterologischen, endokrinologischen, onkologischen, nephrologischen und kardiologischen Erkrankungen finden. Hinzu kommt die breite Spanne der medikamenteninduzierten sekundären Osteoporosen (Mirza und Canalis 2015).

Eine letzte mögliche Variante der Einteilung richtet sich nach dem vorliegenden Schweregrad. Dabei werden vor allem die Ergebnisse einer Knochendichtemessung berücksichtigt. Als normaler Knochen, wird ein Ergebnis gewertet, wenn die Knochendichte weniger als eine Standardabweichung vom statistischen Mittelwert der maximalen Knochendichte gesunder junger Erwachsener abweicht (T-Score). Osteopenie ist definiert als negativ abweichende Standardabweichung von mehr als eins aber weniger als 2,5 vom Mittelwert maximaler Knochendichte. Von Osteoporose spricht man, wenn eine negative Standardabweichung von mehr als 2,5 vorliegt. Man unterscheidet dann weiterhin die präklinische Osteoporose ohne vorausgegangene Frakturen von der manifesten Osteoporose, bei der bereits osteoporosebedingte Frakturen aufgetreten sind (Bartl 2011; Feng und McDonald 2011; Tu et al. 2018).

Aktuell ist die Einteilung rein nach Knochendichtemessungsergebnis international zugunsten einer individuellen Risikobestimmung für eine Fraktur verlassen worden (DVO 2014; Kanis et al. 2014).

\subsubsection{Physiologie des Knochens}

Das menschliche Skelett besteht aus 220 Knochen, die für den Körper vielfältige Aufgaben übernehmen. Sie erfüllen eine Stütz- und Fortbewegungsfunktion, schützen lebenswichtige Organe, wie Gehirn, Herz und Lunge vor äußeren Einwirkungen, Beherbergen das Knochenmark mit dem blutbildenden System und 
fungieren weiterhin als Mineraldepot des Körpers. Der Knochen dient als Speicher für $99 \%$ des Kalziums, $85 \%$ des Phosphats und $50 \%$ des Magnesiums.

Etwa die Hälfte des Knochens besteht aus anorganischen Materialien, die andere Hälfte teilt sich gleichmäßig in Wasser und organische Matrix auf. Letztere wird zu $90 \%$ von Kollagen Typ I gebildet.

Die Gesamtmasse des Skeletts beträgt durchschnittlich $10 \mathrm{~kg} .80 \%$ werden von der Kompakta und nur $20 \%$ von der Spongiosa ausgemacht, welche dafür eine zehnfach größere Oberfläche vorweist. Dies liegt darin begründet, dass die Spongiosa vor allem das axiale Skelett, einschließlich der Wirbelkörper, bildet, die eine verhältnismäßig große Oberfläche aufweisen. Die Röhrenknochen des peripheren Skeletts hingegen bestehen zum Großteil aus kompaktem Knochen (Marx 2012). Während Kindheit und Jugend nimmt die Knochenmasse ständig zu und erreicht ihren Höhepunkt zwischen dem 20. und 30. Lebensjahr. Bis zu diesem Zeitpunkt überwiegt der Knochenaufbau. Dem Zustand der maximalen Knochenmasse, bzw. Knochenmineraldichte wird in der Bewertung osteoporotischer Vorgänge entscheidende Bedeutung beigemessen. Diese sogenannte peak bone mass wird festgelegt als der Wert eines knochengesunden 30-jährigen Erwachsenen und entspricht definitionsgemäß einer Knochenmineraldichte von $100 \%$. Verschiedene Faktoren beeinflussen die peak bone mass, darunter körperliche Aktivität und Ernährungsgewohnheiten, das Körpergewicht, sowie ethnische Zugehörigkeit und genetische Disposition. (Heaney et al. 2000) Zwillingsstudien zufolge können bis zu 85 \% der Unterschiede in der Höhe der peak bone mass durch genetische Faktoren erklärt werden (Stewart und Ralston 2000; Genant et al. 2013).

Nach Erreichen dieses Höhepunktes beginnt ein stetiger Abfall der Knochenmineraldichte. In den ersten fünf Jahren nach der Menopause sinkt die Knochendichte einer Frau um etwa 5 bis $10 \%$. Danach verbleibt der altersassoziierte Verlust an Knochenmasse von 0,5 bis $1 \%$ pro Jahr (Bernstein et al. 2003).

\subsubsection{Pathophysiologie der Osteoporose}

Der Knochen ist ein dynamisches Gewebe mit komplexer Struktur, hoher Durchblutung und Stoffwechselaktivität. Er unterliegt ständigen Umbauprozessen (remodelling). Diese nehmen durch die Mobilisation von Kalzium eine maßgebliche Funktion im Rahmen der Kalziumhomöostase des Körpers ein. Weiterhin dienen sie dem Ersatz von altem Knochengewebe, der Reparatur von geschädigtem Knochen, inklusive kleinster, das Frakturrisiko mitbestimmender Perforationsbrü- 
che der Knochenbälkchen, genannt Mikrofrakturen, sowie der Adaption an wechselnde Belastungsanforderungen (Bartl 2011).

Knochenbildungs- und Knochenresorptionsvorgänge geschehen in den sogenannten Basic Multicellular Units (BMU), von denen der Körper zwei bis fünf Millionen besitzt, und werden reguliert durch zwei gegensätzlich arbeitende Zellen, die Osteoblasten und die Osteoklasten. Während die Osteoklasten mehrkernige Riesenzellen sind, die sich von Monozyten ableiten und proteolytische Enzyme zur Resorption von mineralischer Knochensubstanz sezernieren, entstammen die Osteoblasten einer mesenchymalen Zellreihe. Ihre Funktion liegt vor allem in der Synthese organischer Knochenmatrix, dem Osteoid. Die Aktivität von Osteoblasten und Osteoklasten ist eng aneinandergekoppelt. Bei gesteigerter Resorption des Knochens durch Osteoklasten steigt kompensatorisch die Osteoblastenaktivität und umgekehrt (Rossini et al. 2016; Tu et al. 2018).

Der Zyklus des remodelling dauert etwa drei bis fünf Monate und wird durch die Sekretion verschiedener Wachstumsfaktoren und Zytokine gesteuert. Die Osteoklasten besitzen außerdem Östrogenrezeptoren. Die Anwesenheit von Östrogen unterdrückt die Rekrutierung der Osteoklasten, was das vermehrte Auftreten osteoporotischer Knochenbrüche bei postmenopausalen Frauen erklärt. Der Mangel an Östrogen nach der Menopause resultiert in erhöhter der Osteoklastenrekrutierung. Die verstärkte Aktivität der Osteoklasten führt zu einer kompensatorisch vermehrten, jedoch meist unzureichenden Knochenneubildung durch die Osteoblasten. Eine negative Knochenbilanz ist die Folge (Bernstein et al. 2003).

Diese Art des Verlustes von Knochenmasse wird den High-TurnoverOsteoporosen zugeordnet und steht beispielsweise der glukokortikoidinduzierten Osteoporose gegenüber, die meist einer Low-Turnover-Osteoporose entspricht, bei der eine normale Knochenresorption von reduzierter Knochenneubildung begleitet wird.

\subsubsection{Diagnostik der Osteoporose}

Zur Verhütung von Knochenbrüchen kommt der Diagnostik im Umgang mit der Osteoporose elementare Bedeutung zu. Ziel ist das Erkennen von vorhandenen Risikofaktoren und die Verhütung des Auftretens von Frakturen durch die frühe Einleitung von therapeutischen Interventionen.

Das 10-Jahres-Frakturrsiko wird durch die initiale Erhebung anamnestischer und klinischer Risikofaktoren abgeschätzt. Zur Vorhersage dieses Frakturrisikos wur- 
den zusätzlich zur vormals entscheidenden Knochendichtemessung Risikofaktoren für Frakturen aus großen epidemiologischen Studien integriert (Ismail et al. 2001; DVO 2014).

Tabelle 1: Indikation zur Basisdiagnostik anhand der DVO-Leitlinie 2014

\begin{tabular}{|c|c|c|c|}
\hline $\begin{array}{l}\text { Indikation zur Basisdiagnostik anhand der DVO-Leitlinie } 2014 \text { zur Prophy- } \\
\text { laxe, Diagnostik und Therapie der Osteoporose bei postmenopausalen } \\
\text { Frauen }\end{array}$ & 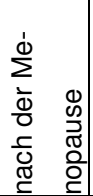 & $\begin{array}{l}3 \\
\dot{8} \\
\varepsilon \\
0 \\
0 \\
0 \\
0\end{array}$ & $\begin{array}{l}3 \\
\dot{R} \\
\varepsilon \\
\varepsilon \\
0 \\
0 \\
0\end{array}$ \\
\hline Niedrigtraumatische singuläre Wirbelkörperfraktur 2. - 3. Grades *2) & $\mathrm{X}$ & $\mathrm{X}$ & \multirow{31}{*}{ 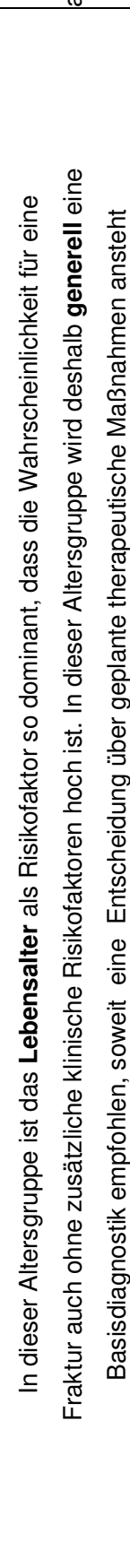 } \\
\hline $\begin{array}{l}\text { Niedrigtraumatische singuläre klinisch manifeste Wirbelkörperfraktur 1. Grades mit Deckplat- } \\
\text { tenimpression }{ }^{* 2)}\end{array}$ & $\mathrm{x}$ & $\mathrm{X}$ & \\
\hline $\begin{array}{l}\text { Niedrigtraumatische singuläre Wirbelkörperfraktur 1. Grades mit Deckplatten-impression } \\
\text { ohne Klinik als Einzelfallentscheidung }{ }^{* 1,2)}\end{array}$ & $x$ & $X$ & \\
\hline Niedrigtraumatische multiple Wirbelkörperfrakturen 1.-3.Grades *2) & $\mathrm{X}$ & $\mathrm{X}$ & \\
\hline $\begin{array}{l}\text { Niedrigtraumatische nichtvertebrale Frakturen } \\
\text { mit Ausnahme von Finger-, Zehen-, Schädel- u. Knöchelfrakturen }\end{array}$ & $\mathrm{x}$ & $\mathrm{X}$ & \\
\hline Cushing Syndrom oder subklinischer Hyperkortisolismus ${ }^{\star 4)}$ & $\mathrm{X}$ & $\mathrm{X}$ & \\
\hline Primärer Hyperparathyreoidismus ${ }^{4)}$ & $\mathrm{X}$ & $\mathrm{X}$ & \\
\hline Wachstumshormonmangel ${ }^{* 4)}$ & $\mathrm{X}$ & $\mathrm{X}$ & \\
\hline $\begin{array}{l}\text { Bestehende oder geplante orale Glukokortikoidtherapie mit } \geq 2,5 \mathrm{mg} \\
\text { Prednisolonäquivalent tgl. für mehr als } 3 \text { Monate im Jahr *4) }\end{array}$ & $\mathrm{x}$ & $x$ & \\
\hline Epilepsie / Antiepileptika *4) & $\mathrm{X}$ & $\mathrm{X}$ & \\
\hline Monoklonale Gammopathie unklarer Signifikanz & $\mathrm{X}$ & $\mathrm{X}$ & \\
\hline Rheumatoide Arthritis & $\mathrm{X}$ & $\mathrm{X}$ & \\
\hline Diabetes mellitus Typ 1 & $\mathrm{X}$ & $\mathrm{X}$ & \\
\hline Zustand nach B-II-Operation oder Gastrektomie & $\mathrm{X}$ & $\mathrm{X}$ & \\
\hline Spondylitis ankylosans als Einzelfallentscheidung *1) & $\mathrm{X}$ & & \\
\hline Therapie mit Aromatasehemmern als Einzelfallentscheidung $\left.{ }^{\star 1)} 4\right)$ & $\mathrm{x}$ & & \\
\hline Therapie mit Aromatasehemmern *3) 4) & & $\mathrm{X}$ & \\
\hline Glukokortikoide hochdosiert inhalativ ${ }^{* 3 / 4)}$ & & $\mathrm{X}$ & \\
\hline Therapie mit Glitazonen *3) 4) & & $\mathrm{X}$ & \\
\hline Diabetes mellitus Typ $2 * 3$ ) & & $\mathrm{X}$ & \\
\hline Spondylitis ankylosans ${ }^{\star 3)}$ & & $\mathrm{X}$ & \\
\hline Proximale Femurfraktur eines Elternteils ${ }^{* 3)}$ & & $\mathrm{X}$ & \\
\hline Multiple intrinsische Stürze oder erhöhte Sturzneigung *3/4) & & $\mathrm{X}$ & \\
\hline Immobilität *3/4) & & $\mathrm{X}$ & \\
\hline Untergewicht $\left(\mathrm{BMI}<20 \mathrm{~kg} / \mathrm{m}^{2}\right)^{* 3 / 4)}$ & & $\mathrm{X}$ & \\
\hline Nikotinkonsum und / oder COPD *3)4) & & $\mathrm{X}$ & \\
\hline Depression / Antidepressiva *3) 4) & & $\mathrm{X}$ & \\
\hline Hyperthyreose oder subklinische Hyperthyreose, sofern persistierend *3) 4) & & $\mathrm{X}$ & \\
\hline Zöliakie *3) & & $\mathrm{X}$ & \\
\hline Protonenpumpeninhibitoren bei chronischer Einnahme ${ }^{\star 334)}$ & & $\mathrm{X}$ & \\
\hline Herzinsuffizienz *3) 4) & & $\mathrm{X}$ & \\
\hline
\end{tabular}


${ }^{1}$ Begründung der Einzelfallentscheidungen sind pro Untersuchung und Patient zu dokumentieren
${ }^{2}$ sofern andere Ursachen nicht wahrscheinlicher sind
${ }^{3}$ Bei Vorliegen mehrerer Risikofaktoren kann individuell auch bei Frauen ab der Menopause und bei Männern ab dem 60.
Lebensjahr eine Basisdiagnostik erwogen werden.
${ }^{4}$ sofern Risiko aktuell bestehend oder vor weniger als 12-24 Monaten beendet

Die Tabelle veranschaulicht die Indikationsstellung der Einleitung einer Basisdiagnostik bei Frauen anhand der DVO-Leitlinie 2014 zur Prophylaxe, Diagnostik und Therapie der Osteoporose. Nach (Siggelkow und Hadji 2016). Die Verwendung erfolgt mit freundlicher Genehmigung von Prof. Dr. H. Siggelkow.

Tabelle 1 zeigt diese zu berücksichtigenden Risikofaktoren. Die folgende Basisdiagnostik besteht aus Anamnese, klinischem Befund, Knochendichtemessung und Basislabor. Bei klinischen Hinweisen auf osteoporotische Wirbelkörperfrakturen sollte weitere bildgebende Diagnostik stattfinden (DVO 2014; Siggelkow und Hadji 2016).

Der Labordiagnostik fällt vor allem differentialdiagnostische Bedeutung zu. Der Ausschluss sekundärer Osteoporoseursachen kann in vielen Fällen durch das Vorhandensein normwertiger Parameter erfolgen. Anderseits kann das Abweichen spezifischer Parameter vom Normbereich ein Hinweis für das Vorliegen eines Prozesses sein, der die Entstehung einer Osteoporose begünstigt oder bedingt.

Die Röntgenuntersuchung von Brust- und Lendenwirbelsäule in zwei Ebenen dient in erster Linie dem Nachweis prävalenter Wirbelkörperfrakturen, da ein Großteil dieser klinisch stumm verläuft. Eine Wirbelkörperfraktur ist außer durch morphologische Kriterien durch eine mehr als zwanzigprozentige Höhenminderung an Vorder-, Mittel- oder Hinterkante eines Wirbelkörpers definiert (Pfeilschifter 2009). Durchgeführt werden sollte eine Röntgenuntersuchung bei Verdacht auf Vorhandensein einer Osteoporose auch bei auffälligem Größenverlust oder Rückenschmerzen unklarer Genese. Zur frühen Beurteilung des Mineralisationsgrades ist die konventionelle Röntgenaufnahme jedoch nicht geeignet und kann die Knochendichtemessung somit nicht ersetzen, da erst bei einem dreißig- bis vierzigprozentigen Verlust an Knochenmasse eine Demineralisation sichtbar wird (Roth et al. 2013).

Therapieindikation besteht, wenn sich in Zusammenschau aller Risikofaktoren und der erhobenen Befunde einschließlich der Knochendichtemessung ein 10-JahresFrakturrisiko von $\geq 30 \%$ ergibt (DVO 2014; DVO 2017). 


\subsubsection{Prävention und Therapie der Osteoporose}

Die Behandlung osteoporotischer Prozesse ist vielfältig. Präventive und spezifische Maßnahmen müssen eng verzahnt durchgeführt werden. Regelmäßig durchgeführte Therapiekontrollen, sowie die adäquate und individualisierte Schmerzeinstellung sowie ggf. anschließende rehabilitative Maßnahmen nach Frakturereignissen nehmen neben der spezifischen antiresorptiven Therapie eine wichtige Position ein.

\subsubsection{Präventives antiosteoporotisches Vorgehen}

Regelmäßige körperliche Aktivität ist eine wesentliche Säule in der Prävention osteoporotischer Vorgänge und osteoporosebedingter Frakturen. Bewegungsmangel führt zu einer Abnahme von Muskelkraft und neuromuskulärer Funktion. Es resultieren vermehrte Stürze, die in letzter Konsequenz häufig ihrerseits Frakturen und nachfolgende Immobilität bedingen. Mehr als 40 \% der über 80-Jährigen stürzen einmal im Jahr. Mit regelmäßig durchgeführtem Kraft- und Gleichgewichtstraining kann die Muskelmasse und direkt proportional dazu die Knochenmineraldichte gesteigert oder zumindest erhalten und das Sturzrisiko reduziert werden (Hamilton et al. 2010; Oral et al. 2013; Winkelmann et al. 2015).

Zur Vermeidung von Stürzen im Alter dienen weiterhin Basismaßnahmen, wie die Überprüfung des Visus und der kritische Einsatz sturzbegünstigender Medikamente sowie die geeignete Verwendung von Hilfsmitteln wie Rollatoren (Pfeilschifter 2009; DVO 2014; DVO 2017).

Eine balancierte Calciumhomöostase ist essentiell für den Knochenstoffwechsel. Grundvoraussetzung für die enterale Calciumaufnahme ist ein normwertiger Vitamin-D ${ }_{3}$-Spiegel im Serumblut. Die Gesamtcalciumzufuhr pro Tag sollte bei etwa $1000 \mathrm{mg}$ pro Tag liegen und eine tägliche Menge von $1500 \mathrm{mg}$ nicht überschreiten (Steingrimsdottir et al. 2005). Der tägliche Calciumbedarf wird normalerweise über die Nahrung abgedeckt. Der Verzehr beispielsweise von zwei Scheiben Käse und einem Glas Milch sind ausreichend. Zur weiteren Aufnahme von Calcium können auch calciumreiche Mineralwässer getrunken werden, bevor eine Supplementierung mit Tabletten notwendig wird (Amling 2015; Chiodini und Bolland 2018).

Bedingt durch die abnehmende Synthese und Wirkung von Vitamin D im Alter sowie die inadäquate Aufnahme mit der Nahrung resultiert vor allem bei Frauen oberhalb des 65. Lebensjahres ein relativer Vitamin-D-Mangel. Sowohl die Synthese als auch die Wirkung des Vitamin $\mathrm{D}_{3}$ sinken mit zunehmendem Alter. Hinzu 
kommt die in vielen Fällen inadäquate Aufnahme von Vitamin $D_{3}$ mit der Nahrung. Resultierend ist ein relativer Vitamin-D-Mangel, der vor allem bei Frauen ab dem 65. Lebensjahr vermehrt auftritt (Tsai et al. 1984). Der anzustrebende Vitamin-DSpiegel im Serum liegt bei $>75 \mathrm{nmol} / \mathrm{l} \mathrm{bzw}$. $30 \mathrm{ng} / \mathrm{ml}$. Bei ungenügender Sonnenexposition wird daher empfohlen 800-1000 IU Vitamin $D_{3}$ pro Tag zu substituieren (Holick und Chen 2008; Reid et al. 2014; Snellman et al. 2014). Eine Verminderung des Auftretens hüftgelenksnaher Frakturen um bis zu $30 \%$ bei Altersheimbewohnern durch die alleinige Substitution von Calcium und Vitamin D konnte gezeigt werden (Chapuy et al. 1994; Cameron et al. 2018).

\subsubsection{Spezielle antiosteoporotische Therapie}

Zur spezifischen antiosteoporotischen Therapie stehen antiresorptive und osteoanabole Therapieoptionen zur Verfügung.

Bisphosphonate zählen zur Gruppe der antiresorptiven Therapeutika. Zu den in Deutschland verabreichten Präparaten gehören Alendronat, Risedronat, Ibandronat und Zoledronat. Sie werden täglich, wöchentlich oder monatlich oral verabreicht. Ibandronat und Zoledronat sind intravenös applizierbar und können daher auch nur alle drei Monate bzw. einmal jährlich verabreicht werden. Studien ergaben, dass die Anwendung von Bisphosphonaten zur Reduzierung von Wirbelkörperfrakturinzidenzen von etwa $50 \%$ führt und das Risiko für eine Schenkelhalsfraktur bei Osteoporose senken (McClung et al. 2001; Bianchi und Sambrook 2008).

Östrogenrezeptormodulatoren Raloxifen und Bazedoxifen gelten vor allem zur Vermeidung von Wirbelkörperfrakturen geeignet. Es konnte jedoch in einer Subgruppe von Patientinnen mit erhöhtem Frakturrisiko gezeigt werden, dass sich auch die Inzidenz von nichtvertebralen Frakturen durch die Einnahme von Bazedoxifen senken lässt (Silverman et al. 2012).

Der seit 2010 zugelassene RANKL-Inhibitor Denosumab wird alle sechs Monate subkutan verabreicht und ist zur Therapie postmenopausaler Frauen zugelassen. Studiendaten belegen, dass Denosumab zu einer kontinuierlichen Zunahme der Knochenmineraldichte führt und diesbezüglich Standardpräparaten wie Alendronat sogar überlegen ist. Es zeigte sich jedoch ein erhöhtes Risiko für Neoplasien und Infektionen (Sutton und Riche 2012; Horne et al. 2018).

Strontiumranelat ist für Patientinnen mit hohem Frakturrisiko zugelassen, die gegenüber anderen Therapiemöglichkeiten Kontraindikationen oder Unverträglichkei- 
ten vorweisen. Einschränkungen für die Verordnung von Strontiumranelat gibt es bei Patientinnen mit erhöhtem kardiovaskulärem Risiko und thrombotischen Ereignissen (Atteritano et al. 2016). Es ist aktuell in Deutschland nicht mehr verfügbar.

Teriparatid, als osteoanabol wirkende Therapieoption, reduziert das Risiko für eine osteoporotische Fraktur im Vergleich mit Risedronat signifikant (Geusens et al. 2018). Die maximale Therapiedauer mit Teriparatid ist jedoch auf zwei Jahre beschränkt.

\subsection{Scores zur Frakturrisikoevaluation}

Verschiedene Scores zur Evaluation von 10-Jahres-Frakturrisiken sind im nationalen und internationalen Gebrauch. Im Folgenden soll ein Überblick über die vorhandenen Scores, sowie deren Entwicklung und Validierung und die einfließenden Risikofaktoren gegeben werden.

\subsubsection{Nationaler Risikoscore}

\subsubsection{Entwicklung von DVO-Leitlinien}

Das Krankheitsbild der Osteoporose bringt eine vielschichtige Problemstellung mit sich, die ein einheitliches Vorgehen bei der Diagnostik, sowie allgemeingültige Leitlinienempfehlungen zum Umgang mit betroffenen und gefährdeten Patientinnen ebenso erstrebenswert wie erforderlich macht. Zu diesem Zweck entstand im Jahr 2003 erstmals eine S3-Leitlinienversion in Auftrag gegeben und verabschiedet vom Dachverband deutschsprachiger osteologischer Fachgesellschaften (DVO), welcher 1999 gegründet worden war.

Der klinisch stumme Verlauf der Krankheit kombiniert mit dem Fehlen eines geeigneten Tests zum Screening auf Osteoporose resultierten in häufig erst später Diagnose nach dem Auftreten von Frakturen, einhergehend mit sozialmedizinisch relevanten Folgen wie Immobilität und Pflegebedürftigkeit, vor allem ältere Menschen betreffend.

Die Empfehlungen dieser Leitlinien entstanden auf der Basis systematischer Recherche in gängigen Leitliniendatenbanken und dem Internet, sowie der Studie diverser Metaanalysen, Systematic Reviews und randomisierten Kontrollstudien, erhoben zwischen Juni 1996 und 2002. Die Bewertung der wissenschaftlichen Evidenz therapeutischer Studien und die Ableitung des Empfehlungsgrades erfolg- 
te nach den EBM-Kriterien des Scottish Intercollegiate Guidelines Network (SIGN) mit den Evidenz-Levels 1-4 und Empfehlungsgraden A-D.

Erste Ergebnisse wurden bei der Tagung der Deutschen Gesellschaft für Osteologie im März 2002 in Graz vorgestellt und eine Kurzfassung hinterher zur Disposition und Kommentierung im Internet veröffentlicht. Nach Prüfung neu aufgetretener Aspekte und weiterer Überarbeitung folgte die Veröffentlichung der ersten „DVOLeitlinienempfehlung zur postmenopausalen Osteoporose“ im März 2003 in Göttingen (Faßbender et al. 2003).

$\mathrm{Zu}$ verstehen sind diese Leitlinienempfehlungen als evidenzbasiertes Tool zur einfachen Anwendung in der medizinischen Praxis.

Das Gesamtziel der Leitlinie wurde definiert als Herausgabe von Empfehlungen zur Primärprävention osteoporotischer Frakturen und Erfassung von Hochrisikogruppen (Case-Finding). Weiterhin wurde eine kostennutzeneffiziente Diagnostik und Differentialdiagnostik angestrebt. Empfehlungen zur Wahl medikamentöser und nichtmedikamentöser Therapieoptionen sowie spezifischer Pharmakotherapie werden gegeben und Therapiedauer und Verlaufskontrollen strukturiert. Auch sozioökonomische Aspekte wie die Optimierung von Versorgungsabläufen und die Erhaltung von Lebensqualität und Funktionsfähigkeit bei Patientinnen nach Auftreten osteoporotisch bedingter Frakturen stehen im Fokus (DVO 2014).

Die entstandene Leitlinie stellt den Konsens aller relevanten Fachgesellschaften für Osteoporose im deutschsprachigen Raum dar um Diskrepanzen im Vorgehen sowie Fehl- und Unterversorgung zu reduzieren (Baum 2010).

\subsubsection{Erste Aktualisierung der DVO-Leitlinien 2006}

Die erste Aktualisierung der Leitlinien von 2003 wurde am 1. Juni 2006 verabschiedet und beinhaltet Erkenntnisse aus Studiendaten seit Verabschiedung der Leitlinie 2003. In dieser Aktualisierung wurden erstmals die altersbedingte Osteoporose und die postmenopausale Osteoporose aufgrund der fehlenden diagnostischen sowie therapeutischen Konsequenzen einer Unterscheidung zusammengefasst. Erstmalig eingeschlossen in die Empfehlungen der Leitlinie wurden auch Männer ab dem 60. Lebensjahr.

Die Therapieempfehlungen der Leitlinie von 2006 schlossen die seit 2003 neu zugelassenen Medikamente Strontiumranelat, Teriparatid und Ibandronat mit ein (DVO 2006). 
Eine weitere Neuerung war der Wechsel von der Betrachtung des relativen Risikos einzelner Risikofaktoren zu einem absoluten Risiko. Es setzt sich aus der gemeinsamen Betrachtung aller vorliegenden Risikofaktoren zusammen und gibt das individuelle Patientenrisiko für das Auftreten einer Fraktur in einem bestimmten Zeitraum an. Auswirkungen hatte diese Änderung auch auf die Therapieschwelle, die sich abhängig von den einfließenden Risikofaktoren ergibt.

Die neue Leitlinie maß der Diagnostik und Therapie der Patientinnen im höheren Lebensalter größere Bedeutung zu, während das in der vorangegangenen Leitlinie überschätze Frakturrisiko bei jungen Patientinnen an Relevanz abnahm (Faßbender und Stumpf 2006).

\subsubsection{Zweite Aktualisierung der DVO-Leitlinien 2009}

Turnusmäßig erfolgte die nächste Leitlinienaktualisierung auf Basis der DELBIKriterien im Jahr 2009. Weiterhin wurde über den Einsatz von Diagnostik und Therapie anhand des individuellen Frakturrisikos entschieden, wobei die Basisdiagnostik ab einem zwanzigprozentigen 10-Jahres-Frakturrisiko und die spezifische Therapie ab einem dreißigprozentigen Risiko in den nächsten zehn Jahren eine osteoporosebedingte Fraktur zu erleiden erfolgte. Erstmals wurden in dieser Version der Leitlinien auch über die glukokortikoidinduzierte Osteoporose hinaus Empfehlungen zur Osteoporose, begünstigt durch spezifische Risiken, gegeben. Der Geltungsbereich der Leitlinie erweiterte sich auf postmenopausale Frauen und Männer ab dem 60. Lebensjahr, Patienten mit einer oralen Glukokortikoidtherapie von mehr als drei Monaten im Erwachsenenalter sowie Patienten mit einem hohen Risiko für oder einer nachgewiesenen sekundären Osteoporose im Erwachsenenalter.

Zu den unter drittens genannten prädefinierten speziellen Ursachen einer Osteoporose zählten die Therapie mit Glukokortikoiden, ein Cushing-Syndrom (manifest und subklinisch), Vorliegen eines primären Hyperparathyreoidismus (pHPT), Therapie mit Aromatase-Inhibitoren, eine antiandrogene Therapie, Hypogonadismus anderer Genese, die Therapie mit Thiazolidindionen (Glitazone), Rheumatoide Arthritis, eine Epilepsie oder Therapie mit Antiepileptika, Zustand nach B-IIOperation oder Gastrektomie, Diabetes mellitus Typ 1, eine subklinische Hyperthyreose sowie Wachstumshormonmangel bei Hypophyseninsuffizienz (Pfeilschifter 2010). 
Da auf die Inanspruchnahme von externem Sponsoring für die Arbeit an der Leitlinienaktualisierung verzichtet wurde, kam es nicht zur Erstellung einer neuen UltraLangfassung, wie sie im Jahr 2006 erschienen war. Stattdessen entstand eine fünfzigseitige Langfassung als Korrelat zur publizierten Kurzfassung der Leitlinien 2006. Änderungen zur Vorversion wurden in den neuen Leitlinien durch nummerierte Hinweise (E01-E186) gekennzeichnet. Ein Begleitskript erläutert und belegt alle von der Vorläuferversion abweichenden Empfehlungen (Erläuterungen zur DVO-Leitlinie 2009). Bei allen unveränderten Punkten hat die Langfassung der Leitlinie von 2006 weiterhin Gültigkeit. Eine vierseitige Kurzversion ist für den Praxisalltag verfügbar und fasst die wichtigen Empfehlungen der Leitlinie übersichtlich zusammen (DVO 2009).

Explizit abgelehnt wurde der Wechsel zur Verwendung des aus Großbritannien stammenden FRAX-Rechners. Begründet wurde diese Ablehnung unter anderem durch die Nichtberücksichtigung des Einflusses von Wirbelkörperfrakturen (Baum 2010).

\subsubsection{DVO-Leitlinie 2014}

Die nächste Aktualisierung der S3-Leitlinie zur Diagnostik und Therapie der Osteoporose erfolgte im Jahr 2014. Grundaufbau sowie Risikomodell dieser Leitlinienaktualisierung entsprechen der Version von 2009.

Die Leitlinien wurden allerdings um einige Risikofaktoren, die, je nach Alter der Patientin eine sofortige Basisdiagnostik rechtfertigen, erweitert. Zu diesen neu hinzugenommenen Risikofaktoren zählen Diabetes Mellitus Typ II, COPD, Herzinsuffizienz, Zöliakie, monoklonale Gammopathie unklarer Signifikanz und Spondylitis ankylosans.

Die Kriterien für Therapieempfehlungen wurden in der Kurzfassung 2014 wie auch schon in der Version von 2009 graphisch in einer Tabelle zusammengefasst dargestellt. Einfließend in die Entscheidung waren dabei der T-Score, das Lebensalter, das Geschlecht sowie die klinischen Risikofaktoren. Ergänzend zu den Risikofaktoren die an dieser Stelle schon in den Leitlinien 2009 berücksichtigt wurden, kamen nun neue Risikofaktoren hinzu, die eine Verschiebung von alters- und geschlechtsbezogenem Schwellenwert des T-Scores für die Indikation der medikamentösen Therapie um $+0,5$ bzw. $+1,0$ rechtfertigen (Neuerburg et al. 2015). Um einen T-Score von $+0,5$ wurde die Therapieschwelle nun zusätzlich bei allen oben genannten 2014 neu hinzu gekommenen Risikofaktoren ausgenommen des Dia- 
betes Mellitus Typ II und der MGUS erhöht. Eine Anhebung des Therapieschwellenwertes um $+1,0$ des T-Scores wurde laut DVO-Leitlinie 2014 bei oraler Glukokortikoideinnahme in einer Dosierung zwischen 2,5 und 7,5 mg Prednisolonäquivalent täglich, der Erkrankung an Diabetes Mellitus Typ I oder optional dem Vorliegen von drei oder mehr niedrigtraumatischen Frakturen in den letzten zehn Jahren (ausgenommen Finger-, Zehen-, Schädel- und Knöchelfrakturen) notwendig (DVO 2014).

\begin{tabular}{|c|c|c|c|c|c|c|}
\hline \multicolumn{2}{|c|}{ Lebensalter in Jahren } & \multicolumn{5}{|c|}{$\begin{array}{l}\text { T-Score (Nur anwendbar auf DXA-Werte. Die Wirksamkeit einer } \\
\text { medikamentösen Therapie ist für periphere Frakturen bei einem } \\
\text { T-Score > -2,0 nicht sicher belegt) }\end{array}$} \\
\hline Frau & Mann ${ }^{2}$ & $-2,0$ bis $-2,5$ & $-2,5$ bis $-3,0$ & $-3,0$ bis $-3,5$ & $-3,5$ bis $-4,0$ & $<-4,0$ \\
\hline $50-60$ & $60-70$ & Nein & Nein & Nein & Nein & Ja \\
\hline $60-65$ & $70-75$ & Nein & Nein & Nein & Ja & Ja \\
\hline $65-70$ & $75-80$ & Nein & Nein & Ja & Ja & Ja \\
\hline $70-75$ & $80-85$ & Nein & Ja & Ja & Ja & Ja \\
\hline$>75$ & $>85$ & Ja & Ja & Ja & Ja & Ja \\
\hline
\end{tabular}

Abbildung 1:Indikation für eine spezifische medikamentöse Therapie (DVO 2014). Mit freundlicher Genehmigung des DVO.

Als spezifische Therapeutika wurden erstmals Bazedoxifen und Denosumab in die Empfehlungen aufgenommen.

Einige Veränderungen gibt es bei den Empfehlungen zur Primärprophylaxe unter anderem im Bezug auf die Calciumzufuhr (1000 bis maximal $2000 \mathrm{mg}$ ), Sonnenlichtexposition (optimales Ausmaß unklar) sowie die Substitution von Vitamin D (bei hohem Sturz- bzw. Frakturrisiko orale Supplemente von 800-1000 I.E.) (Niedhart 2016).

\subsubsection{Zwischenzeitlich diskutiertes Risikomodell des DVO}

Parallel zur Entstehung der DVO-Leitlinienaktualisierung 2014 entwickelte der DVO 2011 bis 2013 ein neues Frakturrisikomodell. Dieses sollte die Vorzüge der neuen DVO-Leitlinien, wie einer individuellen Frakturrisikobewertung und dem gelegten Fokus auf Wirbelkörper- sowie Hüftfrakturen beibehalten und Möglichkeiten zur Verbesserung der Leitlinien, beispielsweise durch größere Expertise bei Anwendung des Modells auf Männer, umsetzen. 
Da vor allem aktuelle und verlässliche Daten zu Wirbelkörperfrakturen in Deutschland schwer zugänglich sind, bilden Hüftfrakturinzidenzen in Deutschland, Österreich und der Schweiz die Grundlage des neuen Modells. Ausgehend von den Daten zu Hüftfrakturinzidenzen in Deutschland wurde sich altersadjustiert das internationale Verhältnis von Hüftfrakturen zu klinischen Wirbelkörperfrakturen, sowie von klinischen Wirbelkörperfrakturen zu radiographischen Wirbelkörper-frakturen zunutze gemacht um die Wirbelkörperfrakturinzidenz in Deutschland abzuschätzen.

Ziel des diskutierten Risikomodells war die individuelle Abschätzung des Frakturrisikos für Hüft- und radiographische Wirbelkörperfrakturen in Abhängigkeit von Geschlecht und Alter der Patientin sowie der Art und der Anzahl seiner klinischen Risikofaktoren.

Die klinischen Risikofaktoren entsprachen den Risikofaktoren aus der DVOLeitlinie 2014 und wurden untergliedert in moderate (relatives Risiko 1,5), starke (relatives Risiko 3,0) und sehr starke (relatives Risiko 6,0) Risikofaktoren. Es wurde davon ausgegangen, dass in der Bevölkerung durchschnittlich ein moderater klinischer Risikofaktor vorliegt (und dass es zwischen den Risikofaktoren keine Interaktion gibt).

Die Empfehlung zur Durchführung einer Basisdiagnostik wird im neuen Frakturrisikomodell ab einem absoluten 1-Jahres-Frakturrisiko von $2 \%$ ausgesprochen. Bei einer Frakturwahrscheinlichkeit von einem Prozent im Folgejahr kann sie optional durchgeführt werden. Die Schwelle zur Durchführung einer spezifischen Osteoporosetherapie liegt nicht mehr bei $30 \%$ 10-Jahres-Frakturrisiko, sondern wird festgelegt als $3 \%$ 1-Jahres-Frakturrisiko, sofern der T-Score kleiner als -2 ist. Die Mortalität zur Relativierung des Risikos wird im neuen Modell ausgeklammert. Ab einem 1-Jahres-Frakturrisiko von 6 \% gilt die Indikation zur Osteoporosetherapie auch unabhängig eines T-Scores kleiner -2. Dieses Vorgehen hat den Vorteil, dass eine Therapie bei hohem Risiko auch ohne DXA-Messung indiziert sein kann.

Bei durchgeführter DXA-Messung beruht das Modell auf den Ergebnissen der Messwerte des Gesamtfemurs, kann jedoch bei stark abweichenden Werten der Knochendichtemessergebnisse der Wirbelkörper adjustiert werden.

Das beschriebene Modell wurde bisher nicht veröffentlicht und nur auszugsweise auf dem Osteologie Kongress 2014 in München vorgestellt und liegt für diese Ar- 
beit aus privaten Quellen vor und wird mit Einverständnis des Autors verwendet (Glüer 2014).

\subsubsection{DVO-Leitlinie 2017}

Die letzte, aktuellste Aktualisierung der S3-Leitlinie zur Diagnostik und Therapie der Osteoporose erfolgte im Jahr 2017. Der neuesten Leitlinie liegen systematische Literaturrecherchen bis Mai 2016 sowie ein interdisziplinärer Konsensusprozess zugrunde. Anders als in den vorangegangenen Leitlinien ist das Entwicklungs-vorgehen der DVO-Leitlinie 2017 AWMF konform. Neu wurden in die Leitlinie weiterhin Frakturdaten aus Österreich und der Schweiz aufgenommen. Risikofaktoren die bisher in keiner vorangegangenen Leitlinie Berücksichtigung fanden, jedoch nun aufgenommen wurden sind Schizophrenie, apoplektischer Insult, Morbus Alzheimer, Morbus Parkinson und Opioideinnahme.

\subsubsection{Internationale Risikoscores}

\subsubsection{FRAX}

FRAX ist ein von einer WHO-Arbeitsgruppe entwickeltes Rechentool zur Abschätzung des individuellen 10-Jahres-Frakturrisikos unter Berücksichtigung definierter klinischer Risikofaktoren. Die Risikoabschätzung erfolgt optional unter Einbeziehung der Knochendichte am Schenkelhals gemessen mittels DXA. Ergebnis der Eingabe in das Rechentool ist ein länderspezifisches Risiko innerhalb der nächsten zehn Jahre eine hüftnahe Fraktur oder eine größere osteoporosebedingte Fraktur, genannt Major Osteoporotic Fracture (MOF) zu erleiden. Letztere schlieBen hüftnahe Frakturen, klinische Wirbelkörperfrakturen sowie Frakturen an Oberund Unterarm ein (Kanis et al. 2010).

Entwickelt wurde der Algorithmus auf der Grundlage einer Metaanalyse von neun populationsbasierten Studien, die sowohl Knochendichte als auch klinische Risikofaktoren erfassten (Kanis et al. 2007).

Ein Problem in der Erstellung eines international verwendbaren Risikorechners ist die Erkenntnis, dass sich das Frakturrisiko regional stark unterscheidet. Am besten ist diese Tatsache für das Auftreten von hüftnahen Frakturen belegt. Hier ergaben sich in den verschiedenen Ländern bis zu fünfzehnfache Unterschiede in der 10-Jahres-Frakturwahrscheinlichkeit. Basierend auf diesem Sachverhalt und Einbeziehung der länderspezifischen Mortalität wurden Risikogruppen erstellt, die kategorisieren, ob das betreffende Land einer sehr hohen, einer hohen, mittleren oder niedrigen Risikokategorie zuzuordnen ist (Kanis et al. 2002). 
Zwölf verschiedene Faktoren nehmen auf den FRAX-Algorithmus Einfluss: Das Alter, begrenzt auf eine Altersspanne von vierzig bis neunzig Jahren (die Unterbzw. Überschreitung dieser Altersgrenze wird als das nächstmögliche einzugebende Alter gewertet), das Geschlecht, Größe und Gewicht, eingehend als Body Mass Index, eine vorausgegangene Fraktur, eine familiäre Belastung in Form einer Hüftfraktur bei einem Elternteil, der Raucherstatus, die Frage nach regelmäßigem Alkoholkonsum, die Einnahme von Glukokortikoiden, die Erkrankung an rheumatoider Arthritis, sowie das Vorhandensein einer sekundären Osteoporose. Hinzu kommt ggf. die Eingabe des Ergebnisses einer Knochendichtemessung, die sich ausschließlich auf Messungen am Schenkelhals bezieht. Hierbei kann zwischen unterschiedlichen DXA-Geräteherstellern gewählt werden. Es besteht die Möglichkeit den absoluten BMD-Wert (in $\mathrm{g} / \mathrm{cm}^{2}$ ) oder den T-Score gemäß NHANES III Referenzwertdatenbank bei Frauen direkt einzugeben.

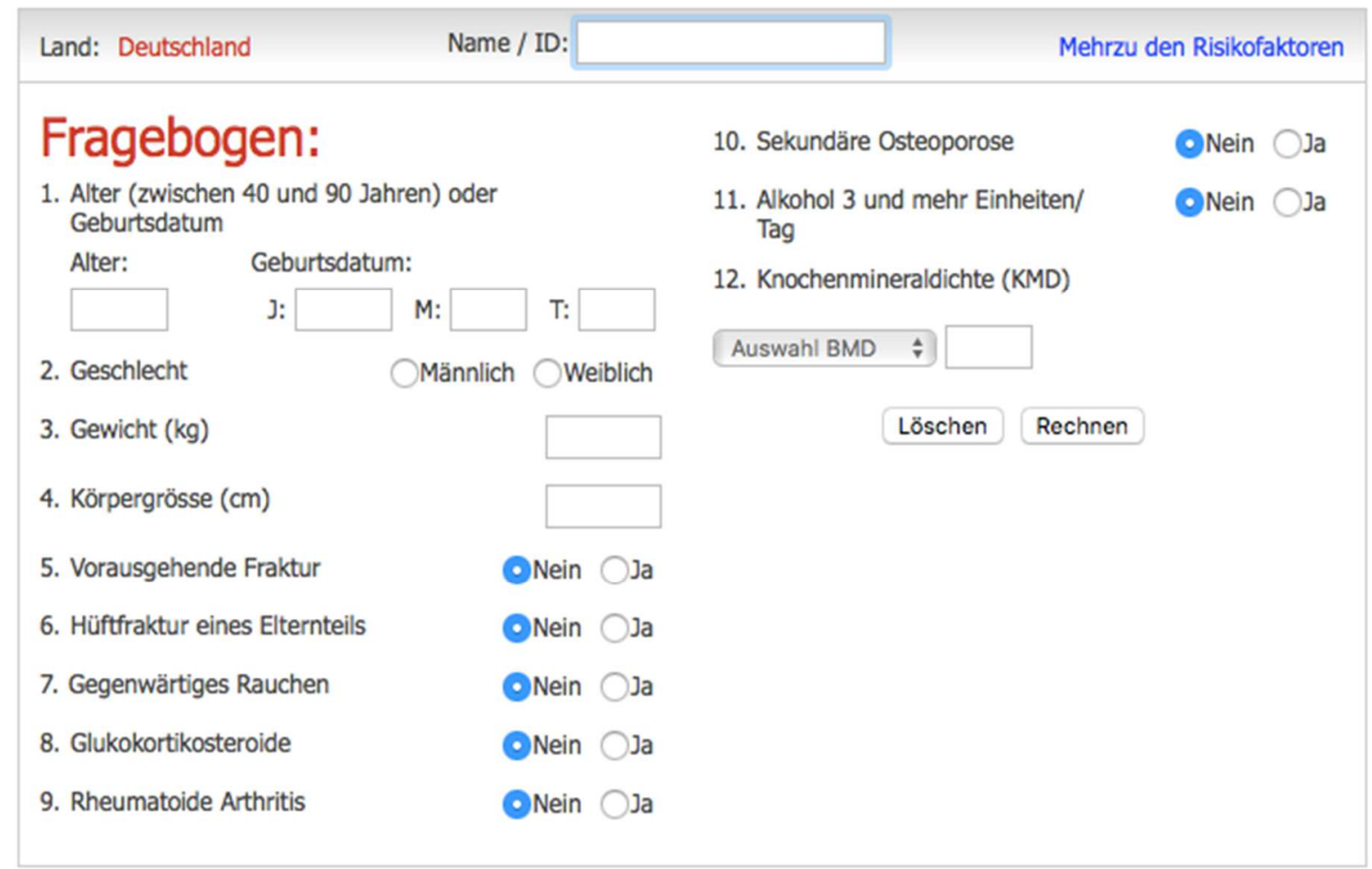

Abbildung 2: FRAX-Eingabemaske (https://www.sheffield.ac.uk/FRAX/tool.aspx?lang=de, besucht am 11.02.2020)

Der FRAX-Risikorechner ist über die Internetseite der Universität Sheffield zugänglich. Vereinfachte Papierversionen basierend auf der Summe der Risikofaktoren können dort ebenfalls heruntergeladen werden. 


\subsubsection{Q-Fracture}

Erstmals in 2009 wurde ein neues Frakturvorhersagetool, genannt Q-Fracture, zur Vorhersage von osteoporotischen Frakturen im Allgemeinen und Hüftfrakturen im Speziellen, publiziert. Q-Fracture wurde zur Benutzung in Großbritannien entwickelt und mittels QResearch database an einer hausärztlichen Primärversorgungspopulation validiert und mit FRAX verglichen. Eine Aktualisierung des Tools mit der Erweiterung der einfließenden Risikofaktoren erfolgte 2012. Zielsetzung bei Entwicklung des Q-Fracture-Scores war unter anderem die Entwicklung eines Tools, das auch ohne Vorhandensein von Laborparametern und Knochendichtemessungsergebnissen in der Primärversorgung aussagekräftig bei der Frakturrisikoabschätzung ist.

\begin{tabular}{|l|}
\hline About you \\
Age (30-99): 64 \\
Sex: $\quad$ Male Female \\
Ethnicity: $\quad$ White or not stated \\
\hline Clinical information \\
Smoking status: non-smoker \\
Alcohol status: none \\
diabetes: none $\quad$ \\
Do either of your parents have osteoporosis/hip fracture? \\
Do you live in a nursing or care home? \\
Have you had a wrist spine hip or shoulder fracture? \\
History of falls? \\
Dementia? \\
Cancer? \\
Asthma or COPD? \\
Heart attack, angina, stroke or TIA \\
Chronic liver disease?
\end{tabular}

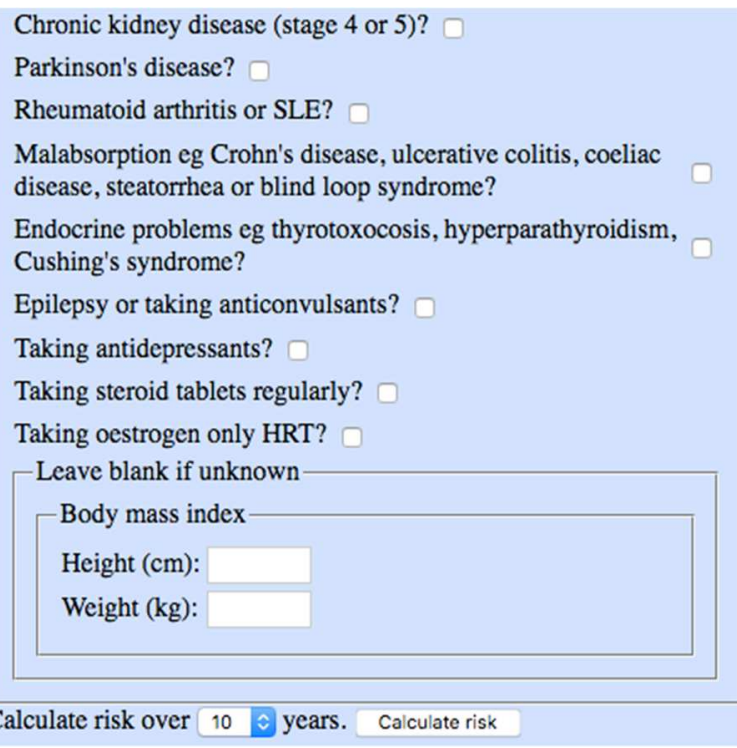

Abbildung 3: Q-Fracture-Eingabemaske (http://www.qfracture.org, besucht am 11.02.2020)

Abbildung 3 zeigt die Eingabemaske des Q-Fracture, erreichbar über die Internetseite www.qfracture.org. Q-Fracture kann für Patienten zwischen dem 30. und dem 99. Lebensjahr verwendet werden. Mittels Q-Fracture kann ein 1- bis 10Jahres-Frakturrisiko für eine Hüftfraktur sowie für eine Hüft-, Wirbelkörper, Schulter- oder Handgelenksfraktur, analog zur MOF des FRAX, erstellt werden. Im Vergleich mit dem FRAX-Tool ist ein größeres Set an Risikofaktoren einfließend, ein Knochendichtemessungsergebnis findet jedoch keine Berücksichtigung. Ein weiterer elementarer Unterschied zum FRAX ist die Nichteinberechnung der Mortalität erstellt wird das Frakturrisiko bei Überleben (Kanis et al. 2016a). 


\subsubsection{Garvan Fracture Risk Calculator}

Der Garvan Fracture Risk Calculator wurde basierend auf Patientendaten der australischen prospektiven Dubbo Osteoporosis Epidemiology Study, die Patientendaten von mehr als 2000 über sechzig-jährigen Patienten einschloss, entwickelt. Er ist über die Homepage des Garvan Institute online abrufbar. Der GarvanRisikorechner erzeugt 5- und 10-Jahres-Risikowerte für das Auftreten einer Hüftfraktur sowie für das Auftreten einer Osteoporotic- bzw. Fragility-Fraktur. Bei der Eingabe berücksichtigt werden Alter und Geschlecht, sowie die Anzahl aufgetretener Frakturen seit dem 50. Lebensjahr, die Anzahl von Stürzen innerhalb der letzten 12 Monate und das Ergebnis einer Knochendichtemessung. Der Garvan Homepage ist zu entnehmen, dass ein „hohes“ Frakturrisiko als ein Risiko über $10 \%$ der 5-Jahres-Frakturwahrscheinlichkeit verstanden wird. Ausgehend von 35bis 50-prozentiger Risikoreduktion durch eine antiosteoporotische Therapie, wird eine wirtschaftlich lohnenswerte Therapieschwelle bei $\geq 10 \%$ 5-JahresFrakturrisiko und $\geq 20 \%$ 10-Jahres-Frakturrisiko angesetzt. 


\section{FRACTURE RISK CALCULATOR}

Fill out the following to estimate your fracture risk

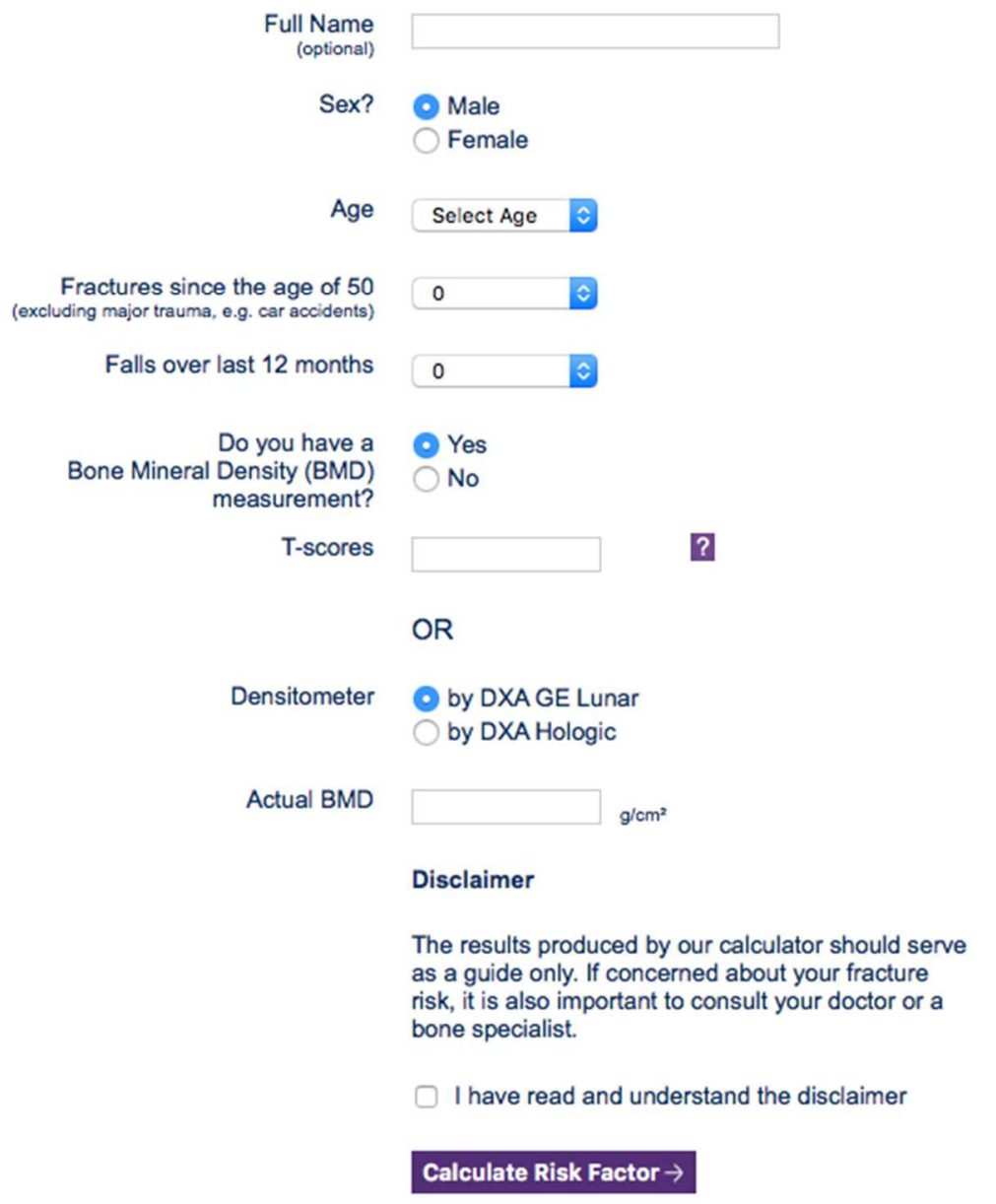

Abbildung 4: Garvan-Eingabemaske (https://www.garvan.org.au/promotions/bone-fracture-risk/calculator/, besucht am 08.10.2019). 


\subsection{Zielsetzung der Arbeit}

Verschiedene Richtlinien und Algorithmen zur Abschätzung des Frakturrisikos bei Patientinnen mit Osteoporose wurden bereits entwickelt und finden in der klinischen Anwendung Gebrauch. Sie unterscheiden sich allerdings nicht nur in der Zusammensetzung einfließender Risikofaktoren sondern auch in der Beurteilung des richtigen Zeitpunkts zum Beginn spezifischer, antiresorptiver Therapiemaßnahmen elementar.

Ziel der Arbeit ist der Vergleich der national angewandten Risikoeinschätzung der DVO-Leitlinie in Deutschland mit dem international verwendeten und an großen Kollektiven validierten FRAX-Risikorechner. Während durch die DVO-Leitlinien eine klare Empfehlung zur Therapie anhand einer Indikationsschwelle gegeben wird, erzeugt der FRAX-Rechner lediglich Frakturwahrscheinlichkeiten. Es soll der DVO-Score mit dem FRAX-Tool verglichen und die Übereinstimmung zwischen den durch die DVO-Therapiegrenze und durch Metaanalysen vorgeschlagene FRAX-Therapiegrenzen eingeschlossenen Patientinnen eruiert werden.

Weiterhin erfolgt ein Vergleich des nichtpublizierten Scores nach C. Gluer, genannt DVO+ hinsichtlich Risikoeinschätzung und Therapieindikationen mit den oben genannten beiden Scores.

Eine weitere Zielsetzung der Arbeit ist es, die Einflussstärke der einzelnen in den FRAX-Rechner eingehenden Risikofaktoren im untersuchten Patientenkollektiv mittels Regressionsanalyse näherungsweise zu eruieren.

Schlussendlich soll ergänzend untersucht werden, inwiefern die Hinzunahme anderer als der laut FRAX berücksichtigten Ursachen einer sekundären Osteoporose Einfluss auf das Ergebnis der 10-Jahres-Frakturwerte des Rechners hat. 


\section{Material und Methoden}

\subsection{Studiendesign}

Bei der vorliegenden Arbeit handelt es sich um eine retrospektive Studie zur Analyse der Daten von Patientinnen mit Osteoporose. Die Patientendaten wurden durch das MVZ endokrinologikum Göttingen erhoben.

\subsection{Probandenrekrutierung}

\subsubsection{Anzahl der Probanden und Dauer der Studie}

Bei den verwendeten Daten handelt es sich um die Daten von Patientinnen, die zwischen Juli 2007 und Juni 2014 erstmalig im MVZ endokrinologikum Göttingen zur Abklärung einer Osteoporose vorstellig wurden. Insgesamt stellten sich in diesem Zeitraum 2021 Patientinnen und Patienten mit dem Verdacht auf das Vorliegen einer Osteoporose vor. 1118 von diesen erklärten sich mit der Nutzung ihrer Daten für diese Studie einverstanden. Ausgeschlossen wurden dann diejenigen, die bereits vor dem 01.07.2007 eine erste Osteoporosediagnostik erfahren hatten. Weiterhin solche, die vom Zeitpunkt der Erstaufnahme keinen Arztbrief vorwiesen und solche, bei denen nach initialer Untersuchung das Vorliegen einer Osteoporose klar ausgeschlossen werden konnte.

Nach Ausschluss der erwähnten Patientengruppe blieb eine Anzahl von 857 Patientinnen und Patienten, davon 147 Männer und 710 Frauen. Die folgende Arbeit soll sich mit der Auswertung der Daten des Frauenanteils des Kollektivs beschäftigen.

Allen 710 Patientinnen des Frauenkollektivs wurde ein Risiko nach DVO-Leitlinie 2009 und 2014 sowie ein Risiko mittels des nichtpublizierten Risikomodells (DVO+) zugeordnet. Bei 675 Patientinnen waren alle für den FRAX-Rechner notwendigen Daten zur Errechnung eines Frakturrisikos ohne BMD-Messung vorhanden. Bei 555 Patientinnen konnte zusätzlich auch noch das Risiko mit BMDMessung erzeugt werden.

\subsubsection{Genehmigung durch die Ethikkommission}

Die Genehmigung der Studie durch die Ethikkommission erfolgte am 18.02.2007. 


\subsection{Ablauf der Aufnahmeuntersuchung}

\subsubsection{Persönliche Daten, klinische Anamnese}

Im Rahmen der Aufnahmeuntersuchung füllten die Patientinnen, die zur Abklärung einer Osteoporose kamen, zwei Fragebögen aus, die den Großteil der relevanten Osteoporoseanamnese erfassen.

Nach selbstständigem Ausfüllen der Bögen wurden diese im Arztgespräch mit den Patientinnen erneut schwerpunktmäßig durchgearbeitet und eventuelle Lücken im Bogen gefüllt.

Es folgte eine generelle Anamneseerhebung, in der vor allem auf die aktuelle Situation der Patientinnen eingegangen wurde. Fragen zum aktuellen Befinden und zu derzeitigen Beschwerden standen im Vordergrund. Bei vorhandenen Schmerzen wurde auf Lokalisation und Schmerzcharakteristik eingegangen. Bei schon stattgehabter Osteoporosediagnostik oder Diagnosestellung wurde die Diagnostik erfragt, vorliegende Befunde wurden besprochen und der Hergang der Diagnosestellung wurde rekapituliert. Es wurden vorausgegangene Stürze und Frakturen registriert und ebenfalls notiert, in welchem Rahmen diese auftraten, traumatisch oder atraumatisch. Die Familienanamnese wurde erfasst und auch weitere, eine Osteoporose begünstigende, Risikofaktoren, wie Nikotin- oder Alkoholkonsum wurden abgeklärt. Auch vorausgegangene Erkrankungen und vergangene sowie die aktuelle Medikation wurden genau erfragt. Weiterhin wurde auf die aktuelle Lebensweise, einschließlich Ernährungsgewohnheiten und ausreichend Bewegung eingegangen.

Alle Punkte sollten in den ausgehändigten Fragebögen schon durch die Patientin notiert worden sein und wurden im Rahmen der Anamnese durch den behandelnden Arzt vervollständigt und ergänzt.

Die folgenden Abbildungen 5 bis 9 zeigen Auszüge aus den Fragebögen. 
Für Frauen

(Zutreffendes bitte ankreuzen)

Erste Monatsblutung mit ........... Jahren

(Datum/Jahr)

Letzte Monatsblutung am

Haben Sie Regelstörungen?

Hatten Sie Geburten?

Wieviel und in welchem Jahr?

Wie lange haben Sie insgesamt gestillt?

(in Monaten)

Nehmen Sie z. Zt. Eine Antibabypille?

Wenn ja, wie lange?

Wurde Gebärmutter entfernt?

Wurden Eierstöcke entfernt?

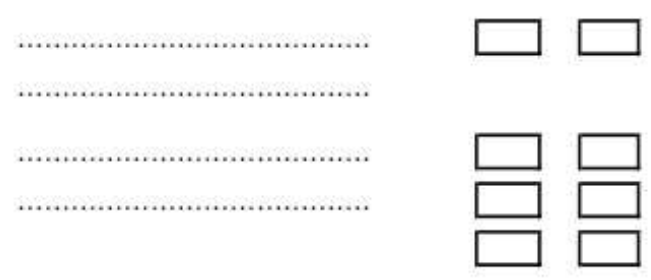

Abbildung 5: Gynäkologische Anamnese (Seite 4 des Patientenfragebogens)

\section{Familienvorgeschichte}

(Eltern, Großeltern, Geschwister der Eltern, eigene Geschwister, eigene Kinder, nicht Sie selbst) Hat oder hatte jemand in Ihrer Familie eine der folgenden Krankheiten:

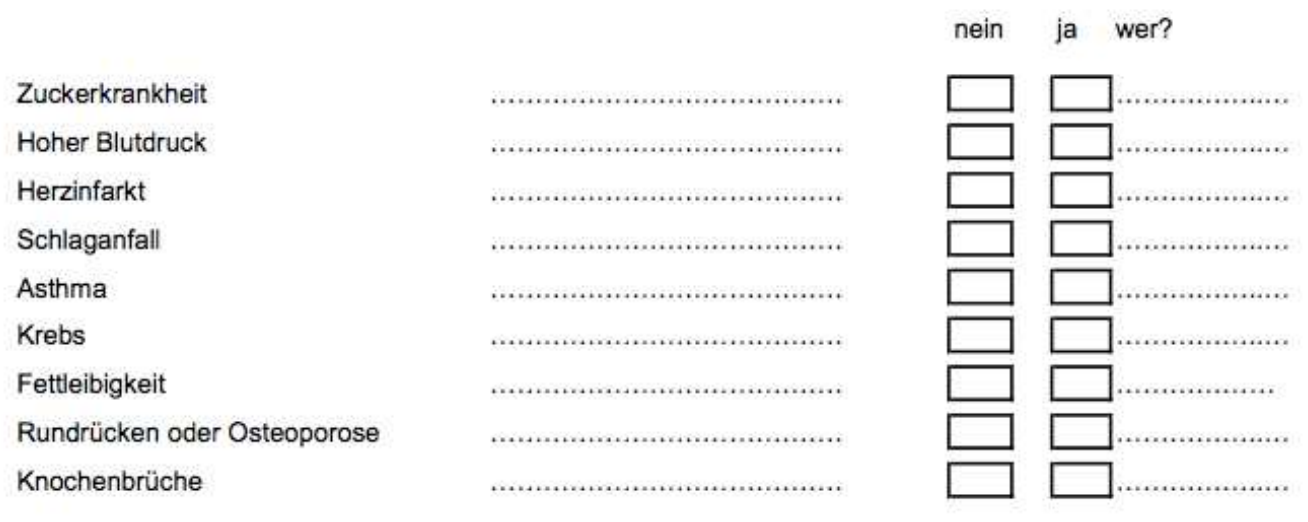

\section{Abbildung 6: Familienanamnese (Seite 4 des Patientenfragebogens)}

5. Medikamentenanamnese:

Namen Sie über längere Zeit folgende Medikamente ein:

$\begin{array}{lll}\begin{array}{l}\text { Cortison } \\ \text { Schlafmittel }\end{array} & \square & \begin{array}{l}\text { Antiepileptika } \\ \text { Marcumarl }\end{array} \\ \begin{array}{l}\text { Sintrom } \\ \text { Schilddrüsen- } \\ \text { hormone }\end{array} & \square & \text { Heparin } \\ & \square & \text { Antirheumatika }\end{array}$

Wenn ja, wie lange?

Vitamin D

Bisphosphonat

Hormone

Wenn ja, wie lange? 
7 - 9 Lebensgewohnheiten:

\begin{tabular}{|l|c|c|c|c|}
\hline Zigarettenkonsum: & keinen & unter 10 Zig. & bis 20 Zig. & über 20 Zig. \\
\hline Alkoholkonsum: & keinen & gelegentlich & bis $2 / 4$ Wein & über 2/4 Wein \\
\hline Diät: & keinen & (fast) vegetarisch & Diabetes & sonstiges \\
\hline Milchkonsum: & keinen & wenig & bis $1 / 4$ tgl. & mehr \\
\hline Käse u. Joghurt: & Keinen & manchmal & öfters & viel \\
\hline Sport: & keinen & fallweise & regelmäßig & viel \\
\hline Immobilität: & nie & 1 Monat & 2 Monate & mehr \\
\hline
\end{tabular}

10 Ihre Körpergröße

a Was war Ihre max. Körpergröße?

b Sind Sie in den letzten Jahren kleiner geworden?

$-^{\prime}--^{m}$

wie viel $\mathrm{cm}$ etwa?

Abbildung 8: Erfassung von Ernährungs- und Lebensgewohnheiten, sowie Registrierung etwaiger Größenabnahme (Seite 2 Osteoporose-Fragebogen)

15 Fragen zu Knochenbrüchen

a Hatten Sie seit dem 50. Lebensjahr einen Knochenbruch? Wenn ja, welchen und wann

Jachenkelhals
Wirbelkörper
Unterarm
Oberarm
Rippe
Unterschenkel

$\square$
mit ............... Nahren
mit .............Jahren
mit ............Jahren
mit .............Jahren
mit .............Jahren
mit ..............Jahren

b Wodurch trat dieser Knochenbruch auf?

im Alltagsleben (z.B. beim Heben, Tragen, Laufen, beim Lagewechsel Ausrutschen, Stolpern u. ä) ohne besondere Ursache

durch Sturz z.B. von der Leiter, vom Stuhl, auf der Treppe, vom Baum durch schweren Sturz

durch Unfall (z. B. Verkehrsunfall) durch einen Unfall

Abbildung 9: Frakturanamnese (Seite 2 Osteoporose-Fragebogen)

\subsubsection{Körperliche Untersuchung}

Der Anamnese folgte eine vollständige klinische Untersuchung, die nicht nur das Gesamtbild der körperlichen Konstitution sondern auch osteoporosespezifische Befunde aufdecken sollte. Die Untersuchung schloss die Ermittlung der Körpermaße sowie eine standardisierte Funktionsprüfung der Wirbelsäule und eine Ermittlung der Mobilität und des individuellen Sturzrisikos ein.

\subsubsection{Laborparameter}

Bei erster Aufnahme der Patientinnen in die endokrinologische Behandlung des MVZ wurde allen Patientinnen ein vollständiges Blutbild abgenommen. Besonderes Merkmal galt im Kollektiv der Osteoporosepatientinnen den Laborparametern, die Auf-, Ab-, und Umbauprozesse des Knochens widerspiegeln. Weiterhin wurde in dieser Untersuchung des Blutes nach auffälligen Parametern gefahndet, die für eine sekundäre Ursache einer Osteoporose sprechen könnten.

Zu den standardmäßig abgenommenen Parametern gehören laut DVO-Leitlinien Blutbild, Blutsenkungsgeschwindigkeit, C-reaktives Protein (CRP), Calcium, Phos- 
phat, Kreatinin, Alkalische Phosphatase (AP), $\mathrm{v}$-Glutamyltransferasen ( $\mathrm{\gamma}-\mathrm{GT})$, Thyroidea-stimulierendes Hormon (TSH) und Eiweiß-Elektrophorese.

\subsection{Osteodensitometrie}

Bei einem auf mindestens $20 \%$ erhöhten Frakturrisiko wird eine Knochendichtemessung durchgeführt. Das von der S3-Leitlinie der DVO empfohlene Standardverfahren stellt die Osteodensitometrie mittels Dual-X-Ray-Absorptiometrie (DXA) an der Lendenwirbelsäule (L1-4) und am proximalem Femur dar (Kurth und Pfeilschifter 2007). Diese erlaubt, anders als weitere bildgebende, das Frakturrisiko abschätzende Verfahren, die Diagnosestellung einer Osteoporose nach WHODefinition.

Zur einheitlichen Verwendung der Knochendichteparameter wurde für alle Patientinnen der minimale T-Score der Femurhalsmessung ermittelt und dieser für die Eingabe in den FRAX-Rechner, die Ermittlung des Frakturrisikos laut DVO+-Score und als Grundlage für eine Osteoporose-Diagnosestellung nach alter WHODefinition verwendet.

\subsection{Erfassung der Daten}

Zur Sammlung aller relevanten Daten wurde eine Exceltabelle auf Grundlage einer alphabetischen Liste aller wegen Osteoporose im Studienzeitraum erstmalig behandelten Patientinnen erstellt.

\subsubsection{Arztbrief}

Aus dem Arztbrief wurden zuerst Informationen zu persönlichen Daten wie Name, Geburtsdatum, Geschlecht, Größe und Gewicht entnommen. Mittels Excel wurde aus den beiden letztgenannten der BMI errechnet und ein etwaiges Untergewicht registriert. Auch die bisherige Größenabnahme wurde aufgenommen. Das Datum der erstmaligen Vorstellung wegen Osteoporose wurde ermittelt. Aus Geburtsdatum und Erstaufnahmedatum wurde das Alter zum Zeitpunkt der Erstvorstellung wegen Osteoporose ermittelt. Zusätzlich wurden die Ergebnisse der neuromuskulären Funktionsuntersuchungen übertragen. Des Weiteren wurden die im Arztbrief angeführten Medikamente sowie vorausgegangene Frakturen und Vorerkrankungen übernommen. 


\subsubsection{Messparameter Knochendichte}

Der DXA-Bogen wurde verwendet, um die Werte der DXA-Messung zu entnehmen, die zeitlich am dichtesten am Erstaufnahmedatum wegen Osteoporose datiert sind.

Dabei wurde unterschieden, ob die DXA-Messung im MVZ endokrinologikum oder in einem anderen Haus vorgenommen wurde. Außerdem wurden an dieser Stelle Messungen mittels Quantitativer Computertomographie (QCT) und die abweichenden Messorte Calcaneus und Arm registriert. Diese konnten im Rahmen der Auswertung nicht berücksichtigt werden.

Notiert wurden die Werte von BMD, T- und Z-Score von der Lendenwirbelsäule sowie von „Femur gesamt" und Femurhälsen auf beiden Seiten. Bei der Messung der Lendenwirbelsäule wurde als regelhafter Messort L1-L4 angesehen. Bei einem Abweichen davon wurde die alternative Lokalisation der Messung notiert.

Mit Excel wurden dann der niedrigste T-Score aller vorhandenen Werte und der minimale T-Score der Femurhalsmessung ermittelt.

\subsubsection{Fragebögen}

\subsubsection{Osteoporose-Fragebogen}

Aus dem im Rahmen der Aufnahmeuntersuchung von den Patientinnen selbstständig ausgefüllten Fragebogen zur Osteoporose wurden die Familienanamnese, erste Angaben zur gynäkologischen Anamnese und zu Lebensgewohnheiten und Risikofaktoren übernommen.

Es folgte ein Abgleich der vorher notierten Angaben zu Medikamenten und Frakturen aus dem Arztbrief mit den Angaben der Patientinnen im Fragebogen.

\subsection{Familienanamese}

Notiert wurde, ob bereits eine diagnostizierte Osteoporose in der Familie bestand, es Frakturen in der familiären Vorgeschichte gab und ob Femurfrakturen im Speziellen in der Familie aufgetreten waren. Verschlüsselt wurden die Angaben als „1“ entsprechend „ja“, „2“ als „nein“, „3“ als „unbekannt“ und „0“ als „keine Angabe“. Überprüft wurden die hier gemachten Angaben mit den Angaben zur Familienanamnese im Patientenfragebogen und im Arztbrief. 


\subsection{Gynäkologische Anamnese}

Aufgenommen wurden das Alter bei erster Regelblutung (in Jahren), das Alter bei letzter Regelblutung (in Jahren) sowie die daraus berechnete Zyklusdauer (in Jahren). Außerdem wurde notiert, ob sich die betreffende Patientin bereits in der Postmenopause befand und falls ja, die Dauer der Menopause in Jahren berechnet.

\subsection{Lebensgewohnheiten und Risikofaktoren}

Die unter Abbildung 8 dargestellten Ernährungs- und Lebensgewohnheiten wurden verschlüsselt von „1“ entsprechend „am wenigsten“ bis „4“ am meisten“.

\subsection{Frakturanamnese}

Es wurden alle vorausgegangenen Frakturen erfasst. Dabei wurde unterschieden, ob es sich um eine Fraktur der Wirbelsäule, eine periphere Fraktur oder um eine periphere Fraktur, die nach dem 50. Lebensjahr auftrat, handelte. Verschlüsselt wurde erneut ,ja“ als „1“, „nein“ als „2“ sowie „keine Angabe“ als „0“. Notiert wurde außerdem, wo die periphere Fraktur auftrat. Für die spätere Eingabe in den FRAXRechner wurde weiterhin unterteilt in „Fraktur generell“ ,ja“ (,1“) bzw. „nein“ (,2“) und „FRAX-relevante-Frakturen“ „ja“ (,1“) bzw. „nein“ („2“).

Später hinzugefügt wurden als Kriterien für die neuen DVO-Richtlinien von 2014 die Kategorien „Niedrigtraumatische Wirbelkörperfrakturen singulär $>2$. Grades oder multipel >1. Grades" sowie "Niedrigtraumatische multiple periphere Frakturen mit Ausnahme von Knöchel-, Hand-, Finger- und Gesichtsfraktur“ „ja“ (,1“) und „nein" (,2“).

\subsection{Medikamentenanamnese}

Im Osteoporose-Fragebogen wurde explizit nach der Einnahme von Schilddrüsenmedikamenten, Glukokortikoiden, Antiepileptika, Protonenpumpeninhibitoren (PPI), Marcumar, Heparin, Antirheumatika und den Knochenstoffwechsel direkt beeinflussenden Medikamenten wie Calcium, Calcitonin, Fluorid und Vitamin D und Bisphosphonaten gefragt.

Verschlüsselt wurden die Angaben nach „1“ als ,ja“, wenn die Einnahme über einen Zeitraum länger als drei Monate erfolgte, oder „2“ als „nein“. Verglichen wurden die Patientenaussagen mit den vorher erfassten Angaben aus dem Arztbrief. Da für die Verwendung der neuen DVO-Leitlinie von 2014 weitere Medikamenteneinnahmen relevant sind, wurden im Nachhinein die Arztbriefe auf die Einnahme 
folgender Medikamente kontrolliert und gegebenenfalls in der Exceltabelle ergänzt: sturzbegünstigende Medikamente (einschließlich Schlafmittel), antiandrogene Medikamente, dopaminerge Medikamente, Schleifendiuretika, Glitazone.

Bei der Einnahme von Glukokortikoiden wurde ergänzt, ob eine inhalative (,1") oder systemische (,2") Einnahme erfolgte, ob eine Therapie mit Glukokortikoiden $>7,5 \mathrm{mg}$ bestand oder geplant war und wie hoch die Dosierung bei bestehender Therapie zum Erfassungszeitpunkt war (in $\mathrm{mg}$ ).

\subsubsection{Patientenfragebogen}

Mittels des Patientenfragebogens wurden die Angaben der Patientinnen zu Vorerkrankungen mit denen aus dem Arztbrief abgeglichen. Im Zweifel vertrauten wir, wie auch bei Differenzen zwischen Arztbrief und Osteoporose-Fragebogen, auf die Aussagen des Arztbriefes, sofern sich die divergierenden Aussagen nicht mittels späterer Arztbriefe noch verifizieren ließen.

Ergänzt wurden mittels Patientenfragebogen weiterhin die Angaben zur gynäkologischen Anamnese um die Frage nach einer Stillanamnese, Hysterektomie, Ovarektomie, Pilleneinnahme sowie Hormontherapie der Wechseljahre, und die Antworten mit „,“ für „ja“, „2“ für „nein“ sowie „3“ für „unbekannt“ verschlüsselt und jeweils die Dauer in Monaten bzw. Jahren notiert.

Des Weiteren wurde im Nachhinein für die Auswertung mit dem neuen DVO-Score im Patientenfragebogen nach Angaben zu vorausgegangenen Stürzen gesucht.

\subsubsection{Laborparameter}

Zum Schluss wurden die Laborergebnisse des Erstaufnahmedatums aufgerufen und notiert. Zur statistischen Auswertung wurden von Desoxypyridinolin (DPD) und Calcium im Urin die Mittelwerte berechnet.

\subsection{Eingabe in den FRAX-Rechner}

Zur Ermittlung des Frakturrisikos im WHO „Fracture Risk Assessment Tool“, kurz FRAX mussten die benötigten Daten in den Risikorechner auf der Homepage der Universität von Sheffield online eingegeben werden.

Zuerst wurden Kontinent und Land aus dem eingegeben wird gewählt. Dann folgten als erforderliche Daten das Alter, Geschlecht, Gewicht und Köpergröße. Aus letzteren beiden errechnet das Programm den BMI. Weiterhin wurden Angaben zu vorausgegangener Fraktur, Hüftfraktur eines Elternteils, gegenwärtigem Rauchen, Glukokortikoideinnahme, rheumatoider Arthritis, dem Vorliegen einer sekundären 
Osteoporose sowie übermäßigem Alkoholkonsum (mehr als drei Einheiten täglich) benötigt. (Auswahlmöglichkeit jeweils „ja“ oder „nein“). Zuletzt wurde die Knochenmineraldichte abgefragt.

Wir entnahmen Alter, Geschlecht, Gewicht und Größe direkt aus der Exceltabelle. Der FRAX-Rechner berücksichtigt Patientinnen und Patienten im Alter von 40 bis 90 Jahren. Ein minimales Gewicht von $25 \mathrm{~kg}$ und ein maximales Gewicht von $125 \mathrm{~kg}$ sind erforderlich, um ein FRAX-Risiko zu erzeugen. Die Körpergröße muss zwischen 100 und $220 \mathrm{~cm}$ liegen.

Aus unseren Angaben zu vorhandenen Frakturen erstellten wir für die Eingabe in den FRAX-Rechner eine neue Kategorie für Frakturen. Dabei wurden zuerst alle Patientinnen die keine Angaben (,0“) in der Frakturanamnese aufwiesen, der Gruppe „Nein“ („2“, keine Fraktur) zugeordnet. In einem zweiten Schritt wurden Frakturen, bei denen ersichtlich war, dass sie nicht der FRAX-Definition einer relevanten Fraktur entsprechen (spontane oder niedrig traumatische Fraktur; Trauma hätte bei gesundem Menschen nicht zu Fraktur geführt) oder eine für OPO untypische Lokalisation aufwiesen ebenfalls aussortiert, sodass eine weitere Kategorie „FRAX-relevante Fraktur“ entsteht. Nur Patientinnen die in dieser Spalte eine „1“ aufwiesen wurden im FRAX Rechner registriert.

Ähnlich verfuhren wir bei der Hüftfraktur des Elternteils. Wir mussten davon ausgehen, dass alle Patientinnen mit der Angabe „Unbekannt“ (,3“) keine relevante Hüftfraktur in der Familie aufwiesen. Diese Patientinnen wurden daher von uns als „Nein“ in den FRAX-Rechner eingegeben.

Der Faktor „Rauchen“ wurde positiv gewertet, wenn beim Zigarettenkonsum „2“, „3“ oder "4“ angegeben wurde, d.h. schon ein Zigarettenkonsum auch unter zehn Zigaretten vorlag. Beim Alkoholkonsum bezogen wir uns auf den aktuellen Konsum. Da unsere Kriterien nicht mit denen des FRAX-Rechners übereinstimmten, werteten wir alle Angaben ab „3“ (2/4 Einheiten Wein tägl.) als Alkoholkonsum „ja“. Der FRAX-Rechner geht landesabhängig bei Alkoholkonsum vom Verzehr von 8 bis $10 \mathrm{~g}$ Alkohol pro Tag aus. Dies entspricht ca. einem Glas Wein à $120 \mathrm{ml}$.

Wie schon bei den Hüftfrakturen wurden „,“ (,keine Angabe“) oder „3“ („Unbekannt") in allen Kategorien wie „2“ („Nein“) gewertet.

Für die sekundäre Osteoporose wurde aus den vorhandenen Angaben eine weitere Spalte zum generellen Vorliegen einer sekundären Osteoporose erstellt. Die laut FRAX-Tool als sekundäre Osteoporose einfließenden Faktoren sind Typ I (in- 
sulinabhängiger) Diabetes, Osteogenesis imperfecta bei Erwachsenen, langjährige, unbehandelte Hyperthyreose, Hypogonadismus oder frühe Menopause (<45-jährig), chronische Mangelernährung oder Malabsorption und chronische Lebererkrankungen. Da diese Ursachen nicht mit denen von uns primär gelisteten sekundären Osteoporoseursachen des MVZ endokrinologikum übereinstimmten, wurde im Nachhinein für jede Patientin ein weiterer FRAX-Wert, genannt FRAX „endo“ generiert. Für diesen wurden die folgenden sekundäre Osteoporoseursachen als sekundäre Osteoporose ,ja“ gewertet: primärer oder sekundärer Hyperparathyreoidismus, Morbus Crohn, alkoholinduzierte Osteporose, steroidinduzierte Osteoporose, Endokrinopathien, Hypo- oder Hypercalcurie, Antiepileptikaeinnahme, Thyroxineinnahme, genetische Faktoren, Hypogonadismus, Osteogenesis imperfecta, Zustand nach Transplantation, Schwangerschaft, Leberzirrhose, Hyperthyreose, Rheumatoide Arthritis, Metastasen, Vitamin-D-Mangel, lokalisierte Osteoporose, Heparin- oder Marcumareinnahme, Mastozystose. So konnte im Nachhinein untersucht werden, ob das Einfließen unterschiedlicher Osteoporoseursachen einen letztendlichen Einfluss auf den FRAX-Wert und somit die Indikation zur antiosteoporotischen Therapie hat.

Der FRAX-Rechner produziert jeweils ein 10-Jahres-Risiko für das Auftreten einer Hüftfraktur und einer MOF. Zu letzteren zählen die klinische Wirbelfraktur, eine Vorderarm-, Hüft- oder Schulterfraktur. Bei 675 weiblichen Patientinnen waren alle oben genannten Werte vollständig vorhanden, sodass wir für diese Anzahl von Patientinnen diese beiden Risikowerte für den FRAX sowie zusätzlich den FRAX „endo“ erhielten.

Zur Eingabe des Ergebnisses der Knochendichtemessung ermittelten wir bei den Patientinnen mit vorhandener DXA-Messung den minimalen T-Score der Femurhalsmessung und errechneten das Risiko im FRAX-Rechner. Bei 555 Patientinnen waren die Werte vollständig vorhanden, sodass wir bei diesen eine FRAX-Frakturwahrscheinlichkeit mit einfließendem T-Score ermitteln konnten. Bei diesen 555 Patientinnen des Frauenkollektivs erhielten wir also acht vergleichbare 10-Jahres-Frakturwahrscheinlichkeiten (zwei mit und zwei ohne einfließende DXAMessung jeweils für FRAX sowie FRAX „endo“).

Als Therapieschwelle gilt im FRAX ein 10-Jahres-Frakturrisiko für eine MOF von $\geq 20 \%$ bzw. international abweichend von $\geq 14 \%$ sowie ein Risiko für eine HF von $\geq 3 \%$ (DVO 2014; Kanis et al. 2016b). 


\subsection{Risikoevaluation nach der DVO-Leitlinie von 2009}

Um Vergleichbarkeit mit Werten des FRAX und des neuen DVO-Risikomodells zu erzeugen, wurde aus den Arztbriefen entnommen, wie hoch das 10-JahresFrakturrisiko nach DVO-Leitlinien eingeschätzt wird. Der Großteil der Patientinnen wurde nach Veröffentlichung der Leitlinienaktualisierung 2009 evaluiert. Ein verhältnismäßig geringer Anteil des Patientenkollektivs erhielt eine Risikostratifikation auf Grundlage der DVO-Leitlinien 2006, in denen einzig die glukokortikoidinduzierte Osteoporose als sekundäre Osteoporoseursache gelistet war. Auch bei diesen Patientinnen wurde von uns allerdings eine zum Zeitpunkt der Aufnahme schon vorhandene mögliche sekundäre Ursache der Osteoporose aus den Arztbriefen erfasst und ggf. mit in die Auswertung einbezogen, sodass diesbezüglich kein Unterschied zwischen den nach DVO-Leitlinie 2006 und DVO-Leitlinien 2009 bewerteten Patientinnen entstand.

Zu Übersichtlichkeitszwecken wurde im Folgenden einheitlich die DVO-Leitlinie 2009 als Grundlage verwendet. Es handelt sich dabei um ein zweistufiges Modell. Nach Anamnese der klinischen Risikofaktoren wurde entschieden, ob das Risiko eine Wirbelkörper- oder proximale Femurfraktur zu erleiden bei $20 \%$ oder höher lag. 


\section{Anamnestische und klinische Risikofaktoren für osteoporotische Frakturen (siehe Kapitel 7 und 8 der Langfassung)}

Die nachfolgende Tabelle zeigt (jeweils mit " " gekennzeichnet) die klinischen Risikofaktoren, bei denen in Abhängigkeit von Alter und Geschlecht ein 10-Jahres-Frakturrisiko $>20 \%$ für Wirbelkörperfrakturen und proximale Femurfrakturen vorliegt, bzw. möglich ist oder bei denen sich unmittelbare therapeutische Konsequenzen ergeben (z.B. OP-Indikation beim pHPT). Ab einem Alter von 70 J. bei Frauen und von 80 J. bei Männern ist das Risiko generell $>20 \%$.

\begin{tabular}{|c|c|c|c|}
\hline Frauen & $<50$ Jahre & 50-60 Jahre & 60-70 Jahre \\
\hline Männer & $<60$ Jahre & 60-70 Jahre & 70-80 Jahre \\
\hline $\begin{array}{l}\text { Sinquläre Wirbelkörperfraktur 2.-3. Grades } \\
\text { (d.h. } 25-40 \% \text { bzw. }>40 \% \text { Höhenminderung) }\end{array}$ & $+(D)$ & $+(A)$ & $+(\mathrm{A})$ \\
\hline Multiple Wirbelkörperfrakturen 1.-3. Grades & $+(D)$ & $+(\mathrm{A})$ & $+(\mathrm{A})$ \\
\hline Orale Glukokortikoide $\geq 7,5 \mathrm{mg}$ Prednisolonäquivalent $\geq 3$ Monate* & $+(A)$ & $+(A)$ & $+(A)$ \\
\hline Cushing-Syndrom* & $+(\mathrm{B})$ & $+(\mathrm{B})$ & $+(\mathrm{A})$ \\
\hline Subklinischer Hyperkortisolismus* & $+(\mathrm{D})$ & $+(\mathrm{D})$ & $+(\mathrm{B})$ \\
\hline Primärer Hyperparathyreoidismus (pHPT)* & $+(\mathrm{B})$ & $+(\mathrm{B})$ & $+(\mathrm{B})$ \\
\hline Singuläre Wirbelkörperfraktur 1. Grades (d.h. 20-25\% Höhenminderung) & *** & ** & $+(\mathrm{A})$ \\
\hline Orale Glukokortikoide $<7,5 \mathrm{mg}$ Prednisolonäquivalent $\geq 3$ Monate * & & $+(\mathrm{A})$ & $+(A)$ \\
\hline Therapie mit Glitazonen bei Frauen* & & $+(D)$ & $+(A)$ \\
\hline Wachstumshormonmangel bei Hypophyseninsuffizienz & & $+(B)$ & $+(B)$ \\
\hline Nichtvertebrale Fraktur(en) nach dem 50. Lebensjahr & & $* *$ & $+(A)$ \\
\hline Therapie mit Aromatasehemmerrn* & & *** & $+(\mathrm{A})$ \\
\hline Antiandrogene Therapie* & & ** & $+(\mathrm{A})$ \\
\hline Rheumatoide Arthritis & & ** & $+(A)$ \\
\hline Proximale Femurfraktur eines Elternteils & & & $+(B)$ \\
\hline Untergewicht $(\mathrm{BMI}<20)^{*}$ & & & $+(A)$ \\
\hline Nikotinkonsum* & & & $+(\mathrm{A})$ \\
\hline Multiple Stürze* & & & $+(\mathrm{A})$ \\
\hline Immobilität* & & & $+(A-B)$ \\
\hline Epilepsie / Antiepileptika* & & & $+(\mathrm{A})$ \\
\hline Zustand nach B-II-Operation oder Gastrektomie & & & $+(A)$ \\
\hline Diabetes mellitus Typ 1 & & & $+(A)$ \\
\hline TSH-Werte $<0,3 \mathrm{mU} / 1^{*}$ & & & $+(\mathrm{B})$ \\
\hline $\begin{array}{l}\text { Sturzbegünstigende Medikamente (Sedativa, Orthostase-verursachend, } \\
\text { Antidepressiva)* }\end{array}$ & & & $+(B-D)$ \\
\hline
\end{tabular}

wenn ${ }_{n}+$ " oder wenn Alter $>70$ Jahre Frau $(\mathrm{A})$ oder $>80$ Jahre Mann $(\mathrm{A}) \rightarrow$ Indikation zur Basisdiagnostik gegeben (sofern damit verbundene therapeutische Maßnahmen umgesetzt werden können).

AuBerhalb dieser Konstellationen wird, von seltenen Ausnahmen abgesehen, derzeit keine Diagnostik empfohlen (D).

Bei einem Beratungsanlass aufgrund der Erbringung technischer Befunde außerhalb der DVO-Empfehlungen in Form von Knochendichtemessverfahren, quantitativem Ultraschall oder Knochenmarkern wird bezüglich der Risikoabschätzung und der evtl. Notwendigkeit der Durchführung einer Basisdiagnostik auf die Langfassung verwiesen.

Abbildung 10: Indikation für eine spezifische Diagnostik der Osteoporose (DVO 2009). Mit freundlicher Genehmigung des DVO.

War dies der Fall wurde eine Basisdiagnostik eingeleitet, welche aus Anamnese, klinischem Befund, einer DXA-Knochendichtemessung und ggf. einem Basislabor sowie einer bildgebenden Diagnostik zur Überprüfung prävalenter Wirbelkörperfrakturen bestand.

Bei Vorliegen eines Risikos > 30 \% für Wirbelkörper- und proximale Femurfrakturen und erniedrigten T-Scores in der DXA-Messung von LWS, Schenkelhals oder proximalem Gesamtfemur bestand eine Indikation für eine spezifische antiosteoporotische Medikation. 


\section{Indikationen für eine spezifische medikamentöse Therapie}

(siehe Kapitel 10.3 der Langfassung)

a. Wirbelkörperfrakturen

Singuläre Wirbelkörperfraktur 2. oder 3. Grades $(25-40 \%$ bzw. $>40 \%)(A)$ oder multiple Wirbelkörperfrakturen 1. bis 3. Grades (A) - unabhängig vom Lebensalter, wenn gleichzeitig ein T-Wert $\leq-2,0$ vorliegt. Rasche Therapie wichtig, da hohes Risiko für weitere Wirbelkörperfrakturen (C).

b. Niedrige Knochendichte in Abhängigkeit von Geschlecht, Lebensalter und weiteren Risikofaktoren*

\begin{tabular}{|c|c|c|c|c|c|c|}
\hline \multicolumn{2}{|c|}{ Lebensalter in Jahren } & \multicolumn{5}{|c|}{$\begin{array}{l}\text { T-Wert } \\
\text { (Nur anwendbar auf DXA-Werte. Die Wirksamkeit einer medikamentösen Therapie ist bei T-Werten >-2,0 nicht belegt) }\end{array}$} \\
\hline Frau & Mann & $-2,0$ bis $-2,5$ & $-2,5$ bis $-3,0$ & $-3,0$ bis $-3,5$ & $-3,5$ bis $-4,0$ & $<-4,0$ \\
\hline $50-60$ & $60-70$ & Nein & Nein & Nein & Nein & Ja \\
\hline $60-65$ & $70-75$ & Nein & Nein & Nein & Ja & Ja \\
\hline $65-70$ & $75-80$ & Nein & Nein & $3 a$ & $3 a$ & $\mathrm{Ja}$ \\
\hline $70-75$ & $80-85$ & Nein & $\mathrm{Ja}$ & Ja & Ja & $\mathrm{Ja}$ \\
\hline$>75$ & $>85$ & Ja & $\mathrm{Ja}$ & $\mathrm{Ja}$ & Ja & Ja \\
\hline
\end{tabular}

*Anhebung der Therapiegrenze um $+0,5-T$-Werte bei einem der folgenden Risikofaktoren (d.h. z.B. auf $-2,5$ statt bei $-3,0$ ), um $+1,0$ T-Werte bei zwei oder mehr der folgenden Risikofaktoren (d.h. z.B. auf $-2,0$ statt bei $-3,0$ ) bis maximal T-Wert $-2,0$

\begin{tabular}{|l|l|}
\hline \multicolumn{2}{|c|}{ Risikofaktoren, die eine Anhebung der Therapiegrenze bedingen } \\
\hline periphere Fraktur nach dem 50. Lebensjahr (B) & TSH $<0,3 \mathrm{mU} / \mathrm{l}$ (falls nicht behebbar) (B) \\
\hline singuläre Wirbelkörperfraktur 1. Grades (B) & Diabetes mellitus Typ 1 (B) \\
\hline proximale Femurfraktur eines Elternteils (B) & Rheumatoide Arthritis** \\
\hline multiple Stürze (B) & B II-Operation / Gastrektomie (B) \\
\hline Immobilitat (B) & Epilepsie (B) \\
\hline Nikotinkonsum (B) & Hypogonadismus (B) (Serumtestosteron $<200 \mathrm{ng} / \mathrm{dl}$ ) \\
\hline subklinischer Hyperkortisolismus (C) & antiandrogene Therapie (B) \\
\hline primärer Hyperparathyreoidismus (konservativ behandelt (B) & Aromatasehemmertherapie** \\
\hline Wachstumshormonmangel bei Hypophyseninsuffizienz (B) & Deutlicher Knochendichteverlust ( $25 \%$ ) am Gesamtfemur über 2 Jahre (B) \\
\hline **siehe auch Langfassung & \\
& \\
c. Glukokortikoide &
\end{tabular}

Orale Glukokortikoide $\geq 7,5 \mathrm{mg}$ Prednisolon-Äquivalent tgl. für 3 oder mehr Monate unabhängig vom Lebensalter, wenn gleichzeitig ein T-Wert $\leq-1,5$ vorliegt $(B)$.

Orale Glukokortikoide $<7,5 \mathrm{mg}$ Prednisolon-Äquivalent tol. für 3 und mehr Monate (B):

Verschiebung der Therapiegrenze der vorstehenden Tabelle um 1,0 T-Wert höher, wenn kein weiterer Risikofaktor vorliegt

um $1,5 \mathrm{~T}$-Werte höher bei einem zusätzlichen Risikofaktor, um 2,0-T-Werte höher bei zwei und mehr zusätzlichen Risikofaktoren bis max, $-2,0$.

Absenken der Therapiegrenze um $-0,5$ bis -1 T-Werte in Abhängigkeit von der klinischen Gesamtsituation möglich (D) d.h. $z$. B. auf $-3,0$ statt bei $-2,5$ )

Abbildung 11: Indikationen für eine spezifische medikamentöse Therapie (DVO 2009). Mit freundlicher Genehmigung des DVO.

Für die Exceltabelle wurde erfasst, ob das Risiko einer 10-Jahres-Fraktur nach DVO-Leitlinie $<20 \%,<30 \%$ oder $>30 \%$ liegt. Zusätzlich wurde in einer weiteren Eingabespalte der Exceldatei notiert, ob nach vorhandener DXA-Messung die Kriterien der Osteoporose erfüllt sind (T-Score $<-2,5)$ oder ob nur eine Osteopenie vorliegt.

\subsection{Risikoermittlung mittels nichtpubliziertem Risikomodell (DVO+)}

Als weitere Variante der Frakturwahrscheinlichkeitsermittlung wurde ein noch nicht veröffentlichtes, von C. Glüer erstelltes Modell angewendet, das auf Grundlage 
der DVO-Leitlinie 2014 erstellt wurde (Glüer 2014). Es wird im Folgenden als DVO+ bezeichnet.

Zur Verwendung des DVO+ war zuerst eine Sichtung notwendig, welche Faktoren erstmalig in der Leitlinie 2014 auftraten und somit in unserem Kollektiv bisher keine Berücksichtigung gefunden hatten. Alle Patientenakten wurden zur genauen Dokumentation der für DVO+ benötigten Risikofaktoren neu geöffnet und die Datentabelle entsprechend ergänzt und nachbearbeitet.

Ein besonderes Augenmerk wurde dabei auf die Frakturanamnese gelegt. Da die bisherige Unterscheidung und Gliederung in die Kategorien „Fraktur generell“, „Fraktur Wirbelkörper“, „Fraktur peripher“ und „Fraktur peripher nach dem 50. Lebensjahr" nicht alle für das Risikomodell nötigen Informationen zu Frakturen lieferte, wurde die Spalte „Fraktur peripher, wo?“ so überarbeitet und vervollständigt, dass entnommen werden konnte, ob es sich um multiple periphere Frakturen vor bzw. nach dem 50. Lebensjahr handelte. Ebenfalls wurde die Kategorie „Niedrigtraumatische multiple periphere Frakturen mit Ausnahme von Knöchel-, Hand-, Finger- und Gesichtsfraktur" aus den nun vorhandenen Angaben erstellt.

Die Frakturrisikofaktoren sind in der Leitlinie 2014 generell in drei Gruppen eingeteilt, je nachdem ob es „Risikofaktoren allgemein“, „Krankheiten“ oder „Medikamente" sind.

Für das neue Risikomodell DVO+ wird weiterhin unterteilt, ob es sich um moderate oder schwere Risikofaktoren handelt. Eine dritte herausgestellte Gruppe beinhaltet Risikofaktoren, die eine „sehr schwere“ Risikoerhöhung bewirken und die nach DVO-Leitlinie 2014 auch bei alleinigem Vorliegen eine sofortige Basisdiagnostik rechtfertigen. 
Tabelle 2: Einfließende Risikofaktoren für Risikomodell DVO+

\begin{tabular}{|c|c|c|c|}
\hline & Moderat & Schwer & Sehr schwer \\
\hline $\begin{array}{c}\text { Risikofaktoren } \\
\text { allgemein }\end{array}$ & $\begin{array}{ll}\text { - } & \text { Singuläre Wirbel- } \\
\text { körperfrakturen } 1^{\circ} \\
\text { mit Deckenplatten- } \\
\text { impression } \\
\text { - } \quad \text { Singuläre nicht ver- } \\
\text { tebrale Frakturen } \\
\text { nach dem 50. Le- } \\
\text { bensjahr } \\
\text { - Proximale Femur- } \\
\text { frakturen bei Vater } \\
\text { oder Mutter } \\
\text { Immobilität } \\
\text { - Untergewicht } \\
\text { - hsCRP } \\
\text { Hyponatriämie }\end{array}$ & $\begin{array}{l}\text { - Zwei nichtverteb- } \\
\text { rale Frakturen } \\
\text { nach dem 50. Le- } \\
\text { bensjahr } \\
\text { - Multiple intrinsi- } \\
\text { sche Stürze }\end{array}$ & 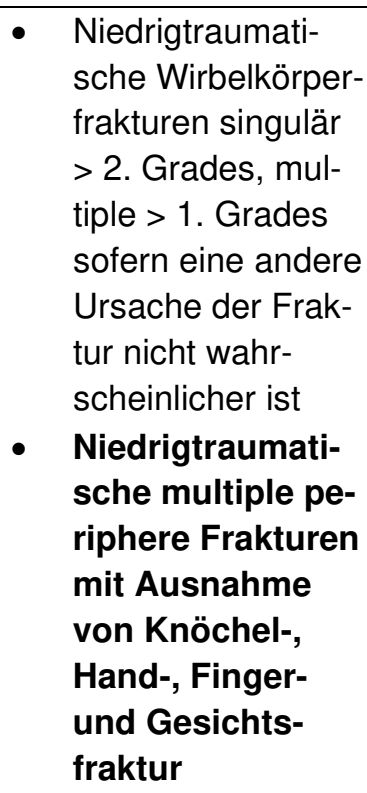 \\
\hline Krankheiten & $\begin{array}{ll}\text { - } & \text { Rheumatoide Arthri- } \\
\text { tis } \\
\text { - Spondylitis anky- } \\
\text { losans } \\
\text { - Zöliakie } \\
\text { - Rauchen und/oder } \\
\text { COPD }\end{array}$ & $\begin{array}{ll}\text { - } & \text { Cushing Syndrom } \\
\text { - } & \text { Subklinischer Hy- } \\
& \text { percortisolismus } \\
\text { - } & \text { Primärer Hyperpa- } \\
\text { rathyreoidismus } \\
\text { - } \text { Wachstumshor- } \\
\text { monmangel } \\
\text { - Subklinische oder } \\
\text { manifeste Hyper- } \\
\text { thyreose } \\
\text { Diabetes mellitus } \\
\text { Typ } 1<70 \text { Jahre } \\
\text { B-II-Resektion, } \\
\text { Gastrektomie } \\
\text { Herzinsuffizienz }\end{array}$ & $\begin{array}{l}\text { Diabetes mellitus } \\
\text { Typ I über } 70 \text { Jah- } \\
\text { re }\end{array}$ \\
\hline Medikamente & $\begin{array}{ll}\text { - } & \text { Antiandrogene The- } \\
& \text { rapie } \\
\text { - } & \text { Aromatasehemmer } \\
\text { - } & \text { Glukokortikoide oral } \\
\text { - } & \text { Glukokortikoide } \\
\text { inhalativ } \\
\text { - } \\
\text { sturzbegünstigende } \\
\text { - } \text { Pedikamente } \\
\text { - } \text { dopaminerge Me- } \\
\text { dikamente } \\
\text { Schleifendiuretika }\end{array}$ & $\begin{array}{ll}\text { - } & \text { Antiepileptika } \\
\text { - } & \text { Glitazone }\end{array}$ & $\begin{array}{l}\text { Bestehende oder } \\
\text { geplante Therapie } \\
\text { mit oralen Gluko- } \\
\text { kortikoiden } \\
>7,5 \text { mg Pred- } \\
\text { nisolonäquivalent } \\
\text { täglich für über } \\
\text { drei Monate }\end{array}$ \\
\hline
\end{tabular}

Unterteilung der in den DVO+ einfließenden Risikofaktoren laut Leitlinie 2014 in die Gruppen „allgemeine Risikofaktoren“, „Krankheiten“ und „Medikamente“. Zur Bewertung des Einflusses auf das Frakturrisiko werden die Risikofaktoren in drei Schweregrade eingeteilt. Die fettgedruckten Faktoren 
sind im Vergleich zur Leitlinie des DVO von 2009 in der Version der Leitlinie des DVO von 2014 neu hinzugekommen (DVO 2014).

Ebenfalls neu in der Leitlinienaktualisierung von 2014 aber im DVO+ nicht berücksichtigt sind die MGUS sowie Diabetes Mellitus Typ II.

Da aus den uns zugänglichen Arztbriefen oft keine Unterscheidungen der Morphologie oder Schwere der Wirbelkörperfrakturen möglich waren, verwendeten wir statt „singuläre Wirbelkörperfrakturen $1^{\circ}$ mit Deckenplattenimpressionen“ alle registrierten singulären Risikofaktoren als moderaten Risikofaktor. Niedrigtraumatische multiple Wirbelkörperfrakturen wurden als sehr schwere Risikofaktoren gewertet.

In der Exceltabelle wurde markiert, ob es sich bei den einfließenden Faktoren um moderate, schwere oder sehr schwere Risikofaktoren handelt und die Risikofaktoren anschließend quantifiziert. Bei den moderaten klinischen Risikofaktoren handelt es sich um Risikofaktoren mit einem relativen Risiko von 1,5 bei den schweren um ein relatives Risiko von drei und bei den sehr schweren um ein relatives Risiko von sechs für das Auftreten von Hüftfrakturen oder radiologischen Wirbelkörperfrakturen.

Zur Auswertung des jährlichen Frakturrisikos nach DVO+ verwendeten wir eine von C. Glüer in seiner Präsentation beim Osteologie Kongress 2014 in München vorgestellte erste Version des eines neuen Risikomodells (Glüer 2014).

Bei der Risikoermittlung wurden die Altersgruppe und die jeweils höchstmögliche Risikokonstellation gewählt, um das vorliegende absolute Risiko und die relative Risikogruppe, die sich aus der Multiplikation des relativen Risikos der beiden höchsten Risikofaktoren ergibt, zu ermitteln. Dies war nötig, da auch andere als in der Tabelle erfasste Konstellationen von Risikofaktoren vorliegen können. Hatte ein Patient beispielsweise vier moderate, zwei schwere und einen sehr schweren Risikofaktor, wurde in der Tabelle die höchstmögliche dieser Risikokonstellationen erfasst. (Im Beispiel also ein schwerer und ein sehr schwerer Faktor). Schlussendlich erhielt jeder Patient also zwei Werte für die 1-JahresFrakturwahrscheinlichkeit. Die relative Risikogruppe und den absoluten aus der Tabelle generierten Wert. Die farbliche Markierung gibt dann eine Empfehlung, ob weitere Diagnostik oder gleich eine Therapie, evtl. in Abhängigkeit vom T-Score erforderlich ist. Generell gilt eine Therapie laut DVO+ als indiziert sobald das 
1-Jahres-Frakturrisiko > $6 \%$ liegt oder bei einem 1-Jahres-Frakturrisiko von $>3 \%$ und einem gemessenem T-Score $<-2$.

\section{Frakturrisiko für Hüft- und rad. WK Frakturen}

\section{in Abh. von Alter sowie Art \& Anzahl kl. Risikofaktoren}

\section{Frauen}

\begin{tabular}{|c|c|c|c|c|c|c|c|c|c} 
relatives Risiko & 1 & 1.5 & 2.3 & 3 & 3.4 & 4.5 & 6 & 9 & 18 \\
\hline Alter \ kl Risikofaktor & 0 & $1 \mathrm{M}$ & $2 \mathrm{M}$ & $1 \mathrm{~S}$ & $3 \mathrm{M}$ & $1 \mathrm{M}$ \& 1 S & $1 \mathrm{SS}$ & $1 \mathrm{M}$ \& 1 SS & 1 S \& 1 SS \\
\hline unter 50 & $<0.2$ & $<0.4$ & $<0.5$ & $<0.7$ & $<0.8$ & $<1.1$ & $<1.4$ & $<2.2$ & $<4.3$ \\
\hline $50-54$ & 0.2 & 0.4 & 0.5 & 0.7 & 0.8 & 1.1 & 1.4 & 2.2 & 4.3 \\
\hline $55-59$ & 0.3 & 0.5 & 0.8 & 1.0 & 1.1 & 1.5 & 2.0 & 3.1 & 6.1 \\
\hline $60-64$ & 0.4 & 0.6 & 0.9 & 1.2 & 1.3 & 1.8 & 2.4 & 3.5 & 7.1 \\
\hline $65-69$ & 0.6 & 0.9 & 1.3 & 1.8 & 2.0 & 2.6 & 3.5 & 5.3 & $>10$ \\
\hline $70-74$ & 1.0 & 1.6 & 2.3 & 3.1 & 3.5 & 4.7 & 6.2 & 9.4 & $>10$ \\
\hline $75-79$ & 1.8 & 2.8 & 4.1 & 5.5 & 6.2 & 8.3 & $>10$ & $>10$ & $>10$ \\
\hline $80-84$ & 3.0 & 4.5 & 6.8 & 9.0 & $>10$ & $>10$ & $>10$ & $>10$ & $>10$ \\
\hline $85-89$ & 4.5 & 6.8 & $>10$ & $>10$ & $>10$ & $>10$ & $>10$ & $>10$ & $>10$ \\
\hline $90+$ & 5.6 & 8.4 & $>10$ & $>10$ & $>10$ & $>10$ & $>10$ & $>10$ & $>10$ \\
\hline
\end{tabular}

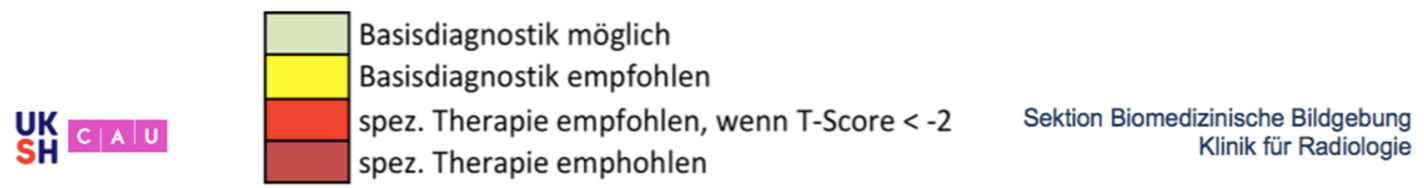

Abbildung 12: Neues Risikoevaluationsmodell, anhand der DVO-Leitlinien 2014 entwickelt. Aufgetragen: Altersgruppen gegen Anzahl und Art der klinischen Risikofaktoren, sowie relatives und absolutes Jahres-Frakturrisiko und resultierende Empfehlung. Mit freundlicher Genehmigung von $\mathbf{C}$. Glüer (Glüer 2014). 


\subsection{Statistische Methoden}

Die statistische Auswertung der vorliegenden Arbeit erfolgte nach einführender Beratung durch die Medizinische Statistik der Universität Göttingen.

Nach Eingabe aller relevanten Daten in eine Microsoft Excel Datei erfolgte die Auswertung durch den Import dieser Datei in das Statistik Programm SPSS Version 23/24/25. Auszählungen einiger Werte wurden noch in Excel vorgenommen, sowie eine Regression zur Analyse der Einflüsse einzelner Risikofaktoren in FRAX durch STATA erstellt.

Mittels deskriptiver Statistik erfolgte die allgemeine Beschreibung des Kollektivs sowie die Analyse der Häufigkeiten einzelner relevanter Frakturrisikofaktoren. Weiterhin erfolgte die Untersuchung auf Normalverteilung der Daten für Alter, Größe, Gewicht sowie der jeweiligen Frakturrisikowerte.

Zum Vergleich verschiedener nicht normalverteilter Parameter wurde der Wilcoxon-Test verwendet. Mittels McNemar-Test erfolgte die Testung der Übereinstimmung der Scores hinsichtlich Therapieempfehlungen.

Zur Aufschlüsselung der Einflüsse der einzelnen Parameter in den FRAX-Rechner wird eine lineare Regression angewendet.

Das Signifikanzniveau der $p$-Werte wird auf $p \leq 0,05\left(^{*}\right)$ als signifikant, sowie $p \leq 0,01$ hochsignifikant $\left(^{* *}\right)$ festgelegt. 


\section{Ergebnisse}

\subsection{Patientenrekrutierung}

Von den 1118 Patientinnen und Patienten, die wegen Osteoporose vorstellig wurden und die Einverständniserklärung zur Teilnahme an dieser Studie gegeben hatten, konnten letztendlich die Daten von 857 Patientinnen und Patienten in der Studie verwendet werden. Der Ausschluss der anderen Patientinnen und Patienten erklärt sich durch das Fehlen von Daten oder Diagnostik oder Behandlung schon vor dem 01.07.2007.

Von den 857 Patientinnen und Patienten gehören 710 dem in dieser Arbeit untersuchten Frauenkollektiv an.

Für den FRAX-Rechner wurde das Kollektiv in weitere Untergruppen eingeteilt. Eine 675 Patientinnen umfassende Gruppe, mit vollständigen Werten zur Errechnung eines FRAX-Risikos ohne BMD, sowie 555 Patientinnen bei denen zusätzlich die Ergebnisse einer Knochendichtemessung vollständig vorlagen und bei denen so auch FRAX-Frakturwahrscheinlichkeiten mit BMD erzeugt werden konnten. Somit standen insgesamt für die Auswertung drei verschieden große Gruppen zur Verfügung. Im folgenden Ergebnisteil werden zur besseren Vergleichbarkeit nur die 555 Patientinnen eingeschlossen, die vollständige Werte zur Eruierung eines FRAX-Risikowertes inklusive Knochendichtemessung vorwiesen.

\subsection{Patientencharakterisierung}

\subsubsection{Basisdaten}

Die unten stehende Tabelle 3 zeigt die Basisdaten der 555 Patientinnen. Sichtbar wird, dass den Risikofaktor mit der im Kollektiv größten Prävalenz die vorausgegangene Fraktur darstellt, die bei über der Hälfte der Patientinnen vorlag. Niedrigste Prävalenz zeigte die Rheumatoide Arthritis (7,03 \%). 
Tabelle 3: Basisdaten der Patientinnen $(n=555)$

\begin{tabular}{c|cc} 
Basisdaten & $\boldsymbol{n}$ & MVZ endokrinologikum Göttingen \\
\hline Alter in Jahren $( \pm$ SD) & 555 & $64,21( \pm 10,3)$ \\
BMI in kg/m² $( \pm$ SD) & 555 & $24,94( \pm 4,66)$ \\
Vorausgegangene Fraktur & 292 & $52,61 \%$ \\
Hüftfraktur eines Elternteils & 77 & $13,87 \%$ \\
Gegenwärtiges Rauchen & 91 & $16,40 \%$ \\
Glukokortikoideinnahme & 93 & $16,76 \%$ \\
Rheumatoide Arthritis & 39 & $7,03 \%$ \\
Alkoholkonsum & 48 & $8,65 \%$ \\
T-Score Femurhals ( \pm SD) & 555 & $-1,86( \pm 0,98)$ \\
Sekundäre Osteoporose (laut FRAX) & 126 & $22,70 \%$ \\
Sekundäre Osteoporose (laut DVO) & 116 & $20,90 \%$
\end{tabular}

\subsubsection{Alter}

Es wurden 555 Patientinnen im Alter von 40 bis 91 Jahren in die Auswertung eingeschlossen. Das durchschnittliche Alter der Patientinnen lag bei 64,21 $( \pm 10,3)$ Jahren. Das Alter war im vorliegenden Kollektiv nicht normalverteilt.

Für die Eingabe und Auswertung des nichtpublizierten Risikomodells DVO+ wurden die Patientinnen in zehn Altersgruppen eingeteilt. Da in dieser Einteilung die höchste Altersgruppe Patientinnen von 90 Jahren und älter einschließen würde, im Frauenkollektiv der 555 Patientinnen jedoch nur zwei Patientinnen in diese Gruppe fallen würden, wurden die beiden höchsten Altersgruppen zusammengefasst (84 Jahre und älter). Tabelle 4 verdeutlicht die Häufigkeitsverteilung in den Altersgruppen.

Tabelle 4: Häufigkeitsverteilung in Altersgruppen nach DVO-Risikomodell

\begin{tabular}{c|cc} 
Alter in neun Gruppen & Anzahl (n) & Prozent \\
\hline unter 50 Jahre & 42 & $7,6 \%$ \\
50-54 Jahre & 61 & $11,0 \%$ \\
55-59 Jahre & 86 & $15,5 \%$ \\
60-64 Jahre & 97 & $17,5 \%$ \\
65-69 Jahre & 86 & $15,5 \%$ \\
70-74 Jahre & 92 & $16,2 \%$ \\
75-79 Jahre & 58 & $10,5 \%$ \\
80-84 Jahre & 17 & $3,1 \%$ \\
über 84 Jahre & 16 & $2,9 \%$ \\
Gesamt & 555 & $100,0 \%$
\end{tabular}




\subsubsection{Größe, Gewicht, BMI}

Die durchschnittliche Größe der 555 Patientinnen betrug 162,2 cm $( \pm 7,2)$ mit einem Maximum von 190,5 cm und einem Minimum von 137,5 cm. Bei der Größe lag eine Normalverteilung vor. Das durchschnittliche Gewicht lag bei $65,9 \mathrm{~kg}$ $( \pm 12,5)$. Es ergab sich ein durchschnittlicher BMI von $24,9 \mathrm{~kg} / \mathrm{m}^{2}( \pm 4,7)$. Gewicht und BMI waren im betrachteten Kollektiv nicht normalverteilt.

Tabelle 5: Basisdaten zu Größe, Gewicht und BMI

\begin{tabular}{c|ccccc} 
& Mittelwert & SD & Maximum & Minimum & Median \\
\hline Größe (cm) & 162,15 & 7,24 & 190,5 & 137,5 & 162,2 \\
Gewicht $(\mathbf{k g})$ & 65,87 & 12,46 & 116,6 & 34,1 & 64 \\
BMI $\left(\mathbf{k g} / \mathbf{m}^{2}\right)$ & 24,94 & 4,66 & 46,95 & 14,53 & 24,39
\end{tabular}

\subsubsection{Knochendichtemessung}

Tabelle 6 zeigt die Knochendichtemessungsergebnisse der verschiedenen Messlokalisationen. Bei allen 555 Patientinnen lag wenigstens ein T-Score am Femurhals vor. Der durchschnittliche T-Score am Femurhals lag bei -1,86 $( \pm 0,98)$ und damit über dem mittleren T-Score der Wirbelsäule $(-2,21 \pm 1,23)$. Alle Lokalisationen zusammengefasst betrachtet lag der durchschnittliche T-Score bei -2,51 $( \pm 0,99)$ und damit noch unter dem mittleren T-Score der Wirbelsäule. 
Tabelle 6: Knochendichtemessungsergebnisse an drei verschiedenen Lokalisationen (Wirbelsäule, Femur gesamt und Femurhals)

\begin{tabular}{|c|c|c|c|c|c|c|}
\hline$B M D$ & $N$ & Mittelwert & $S D$ & Minimum & Maximum & Median \\
\hline BMD Wirbelsäule $\left(\mathrm{g} / \mathrm{cm}^{2}\right)$ & 485 & 0,895 & 0,156 & 0,056 & 1,424 & 0,881 \\
\hline T-Score Wirbelsäule & 500 & $-2,214$ & 1,226 & $-5,1$ & 3,4 & $-2,4$ \\
\hline Z-Score Wirbelsäule & 473 & $-0,893$ & 1,327 & $-7,5$ & 5,5 & -1 \\
\hline BMD Femurhals $\left(\mathrm{g} / \mathrm{cm}^{2}\right)$ & 512 & 0,746 & 0,116 & 0,124 & 1,193 & 0,741 \\
\hline T-Score Femurhals & 555 & $-1,86$ & 0,976 & $-4,5$ & 3,7 & $-1,9$ \\
\hline Z-Score Femurhals & 504 & $-0,54$ & 0,88 & $-3,5$ & 2,2 & $-0,6$ \\
\hline $\begin{array}{l}\text { BMD Femur gesamt } \\
\qquad\left(\mathrm{g} / \mathrm{cm}^{2}\right)\end{array}$ & 518 & 0,779 & 0,118 & 0,178 & 1,277 & 0,776 \\
\hline T-Score Femur gesamt & 540 & $-1,181$ & 0,963 & $-5,4$ & 2,3 & $-1,8$ \\
\hline Z-Score Femur gesamt & 515 & $-0,702$ & 0,925 & $-3,8$ & 2,1 & $-0,7$ \\
\hline $\begin{array}{l}\text { BMD Minimum }\left(\mathrm{g} / \mathrm{cm}^{2}\right) \\
\text { (alle Lokalisationen) }\end{array}$ & 521 & 0,728 & 0,117 & 0,056 & 1,17 & 0,726 \\
\hline $\begin{array}{c}\text { T-Score Minimum (alle } \\
\text { Lokalisationen) }\end{array}$ & 555 & $-2,509$ & 0,994 & $-5,4$ & 3,7 & $-2,6$ \\
\hline $\begin{array}{c}\text { Z-Score Minimum (alle } \\
\text { Lokalisationen) }\end{array}$ & 522 & $-1,257$ & 0,977 & $-7,5$ & 2,1 & $-1,3$ \\
\hline
\end{tabular}

\subsection{0-Jahres-Frakturwahrscheinlichkeiten nach DVO-Leitlinien}

Zur Differenzierung der verschiedenen 10-Jahres-Frakturwahrscheinlichkeiten nach DVO-Leitlinien wurde das Kollektiv der 555 Patientinnen in drei Risikogruppen eingeteilt. Dabei wurde unterschieden, ob ein Risiko unter $<20 \%$, zwischen 20 und $30 \%$ oder ein Risiko > $30 \%$ vorlag, wobei ein Überschreiten der $30 \%$ 10 Jahres-Frakturwahrscheinlichkeit eine Therapieindikation darstellte. Zur Risikogruppe mit einem 10-Jahres-Frakturrisiko von unter $20 \%$ zählten 22 Patientinnen und somit ein prozentualer Anteil am Gesamtkollektiv von 3,96 \%. Zur Risikogruppe der Patientinnen mit mindestens $20 \%$ und höchstens $30 \%$ 10-JahresFrakturrisiko zählten 240 Patientinnen. Dies entspricht 43,24 \%. 293 Patientinnen 
wurde eine 10-Jahres-Frakturwahrscheinlichkeit von $>30 \%$ zugeordnet, einem prozentualen Anteil von 52,79\% entsprechend (siehe Abbildung 13).

\section{Risikogruppeneinteilung DVO}

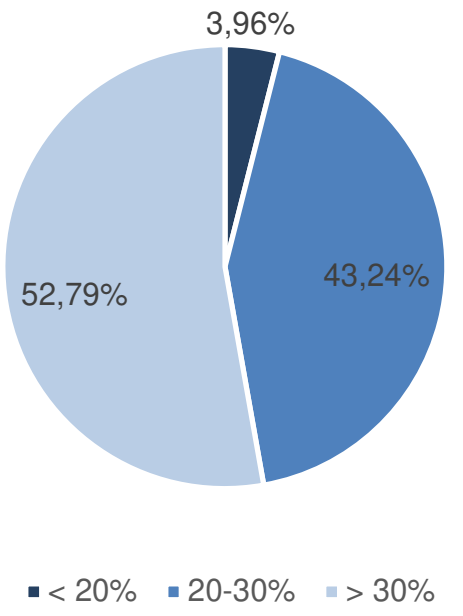

Abbildung 13: Risikogruppeneinteilung nach DVO-Leitlinien

\subsection{Risikomodell DVO+}

\subsubsection{Relative Risikogruppeneinteilung DVO+}

Jede Patientin wurde im nichtpublizierten DVO-Risikomodell DVO+ einer relativen Risikogruppe, bestimmt durch die Anzahl der Risikofaktoren, zugeteilt und bekam zusätzlich ein absolutes 1-Jahres-Frakturrisiko unter Einbeziehung der Risikofaktoren und des Alters zugeordnet. Die untenstehende Tabelle 7 verdeutlicht die Häufigkeitsverteilung der Patientinnen innerhalb der relativen Risikogruppen. Knapp ein Viertel der Patientinnen wies keinen der in den DVO+ einfließenden Risikofaktoren auf. Das absolute Frakturrisiko dieser Patientinnen wird im DVO+ somit durch das Alter definiert. Neun Prozent der Patientinnen hatten ein Risiko, das ohne Einfluss des Alters in die höchste relative Risikogruppe mit $18 \%$ relativem Frakturrisiko fiel. 
Tabelle 7: Häufigkeitsverteilung der relativen Risikowerte im DVO+

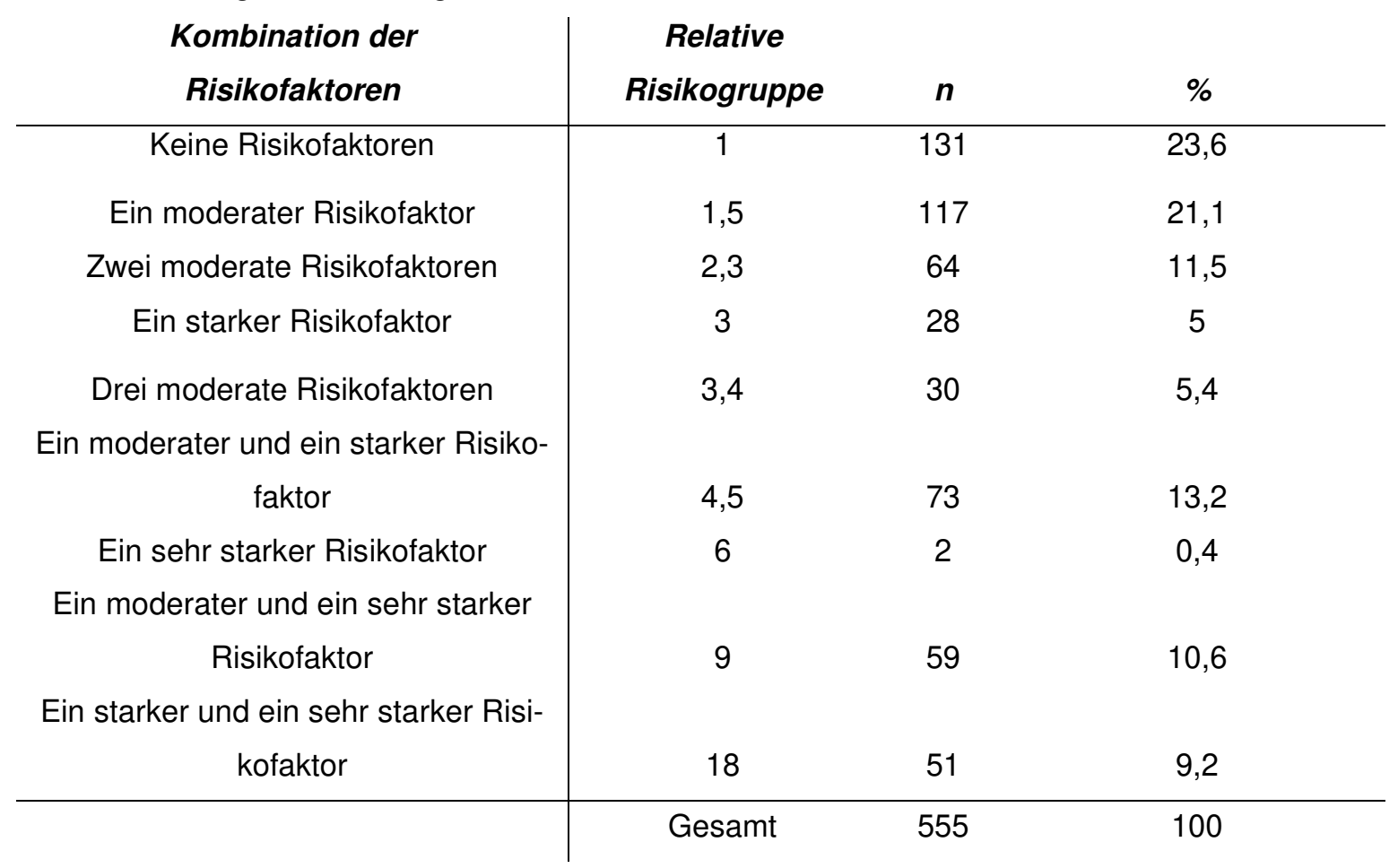

\subsubsection{Absolute Risikowerte DVO+}

Zu beachten bei der Auswertung der absoluten Risikowerte war die Problematik, dass das neue Risikomodell nicht nur mit definierten Risikowerten arbeitet, sondern bei bestimmten Risikokombinationen von einem Risiko "kleiner als“ bzw. „größer als" ausgeht. Bei allen Patientinnen mit einem Alter unter 50 Jahren wird von einem Risiko "kleiner als“ ausgegangen. Weiterhin wird jedes 1-Jahres-Risiko, das größer als zehn Prozent ist, als "größer zehn“ notiert. Um auch diese Werte in die statistische Auswertung mit einbeziehen zu können, vor allem da es sich bei dem Größer-als-10-Anteil um einen beträchtlichen Teil von knapp einem Zehntel des Gesamtkollektivs und die Hochrisikogruppe handelt, wurden alle Kleiner-alsWerte mit dem Abzug von 0,01 Prozentwerten verschlüsselt. Allen Werten größer als zehn wurde ein Hundertstel-Prozentpunkt addiert, sodass diese mit 10,01 verschlüsselt wurden. Auf diesen Sachverhalt und die entstehenden Konsequenzen in der Auswertung wird an entsprechenden Stellen im Diskussionsteil noch einmal hingewiesen.

Es ergab sich ein mittleres 1-Jahres-Frakturrisiko im DVO+ von 2,8\% $( \pm 3,17)$. 
Tabelle 8: 1-Jahres-Frakturrisiko in DVO+

\begin{tabular}{l|ccccc} 
& Mittelwert & SD & Maximum & Minimum & Median \\
\hline 1-Jahres-Frakturrisiko DVO+ & 2,8 & 3,17 & 10,01 & 0,19 & 1,3
\end{tabular}

\subsection{FRAX-Risikoscore}

Mittels FRAX-Tool wurden für jede Patientin 10-Jahres-Risikowerte für eine größere osteoporotische Fraktur, genannt Major Osteoporotic Fracture (MOF), und das Risiko für eine Hüftfraktur, Hip Fracture (HF), generiert. Jede der 555 Patientinnen erhielt folglich vier Risikowerte; zwei mit und zwei ohne Einbeziehung des minimalen Knochendichtemessungsergebnisses am Femurhals.

Die durchschnittliche 10-Jahres-Frakturwahrscheinlichkeit für eine MOF betrug im Kollektiv ohne einfließende Knochendichtemessung 14,18\% ( $\pm 10,47)$. Die Wahrscheinlichkeit für eine HF lag deutlich niedriger bei $6,29 \%( \pm 8,08)$. Mit einbezogener Knochendichtemessung ergaben sich durchschnittliche FRAX-Risikowerte von $13,24 \%( \pm 9,81)$ für eine MOF und $5,39 \%( \pm 7,65)$ für eine HF. Diese Werte lagen demnach niedriger als die Werte ohne Knochendichtemessung. Allerdings lagen die maximalen Ergebnisse des FRAX-Rechners mit $86 \%$ Frakturwahrscheinlichkeit für eine MOF und $84 \%$ für eine HF unter Einbeziehung des TScores deutlich höher als bei der Eingabe ohne Knochendichtemessung. Tabelle 9 zeigt Mittelwerte, Standardabweichung, Maxima, Minima und Mediane in der Übersicht. Die FRAX-Risikowerte folgten keiner Normalverteilung. Die dargestellten Mittelwerte unterscheiden sich untereinander hochsignifikant $(p<0,01)$.

Tabelle 9: FRAX-10-Jahres-Risikowerte aller Patientinnen ( $n=555)$

\begin{tabular}{c|ccccc} 
& Mittelwert & SD & Maximum & Minimum & Median \\
\hline FRAX MOF ohne BMD & 14,18 & 10,47 & 65,0 & 2,1 & 11,0 \\
FRAX HF ohne BMD & 6,29 & 8,08 & 55,0 & 0,1 & 3,6 \\
FRAX MOF mit BMD & 13,24 & 9,81 & 86,0 & 2,0 & 11,0 \\
FRAX HF mit BMD & 5,39 & 7,65 & 84,0 & 0,0 & 2,8
\end{tabular}

Zur Veranschaulichung wurden die FRAX-Risikowerte im Bezug auf die Altersgruppeneinteilung betrachtet. Das durchschnittlich höchste FRAX-Risiko sowohl für eine MOF als auch für eine HF bestand in der Gruppe der über 80-jährigen Patientinnen, unabhängig davon, ob die Knochendichte einbezogen wurde. 
Tabelle 10: FRAX-Mittelwerte innerhalb Altersgruppen nach Risikomodell DVO+

FRAX Mittelwerte

Altersgruppen aus Risikomodell

\begin{tabular}{c|ccccc} 
Altersgruppen aus Risikomodell & & & & & \\
\cline { 5 - 7 } DVO+ & $\boldsymbol{n}$ & MOF & HF & MOF & HF \\
\hline unter 50 Jahre & 42 & 6,5 & 1,45 & 7,36 & 2,48 \\
50-54 Jahre & 61 & 7,24 & 1,7 & 8,89 & 3,17 \\
55-59 Jahre & 86 & 8,32 & 2,25 & 10,24 & 3,73 \\
60-64 Jahre & 87 & 10,16 & 2,84 & 10,74 & 3,3 \\
65-69 Jahre & 96 & 14,99 & 5,81 & 13,25 & 4,54 \\
70-74 Jahre & 82 & 18,08 & 9,43 & 16,75 & 7,88 \\
75-79 Jahre & 58 & 22,62 & 12,16 & 17,5 & 7,98 \\
80-84 Jahre & 17 & 36,35 & 25,04 & 27,17 & 16,01 \\
über 84 Jahre & 16 & 35,63 & 22,45 & 26,19 & 12,74
\end{tabular}

FRAX-Mittelwerte in Altersgruppen

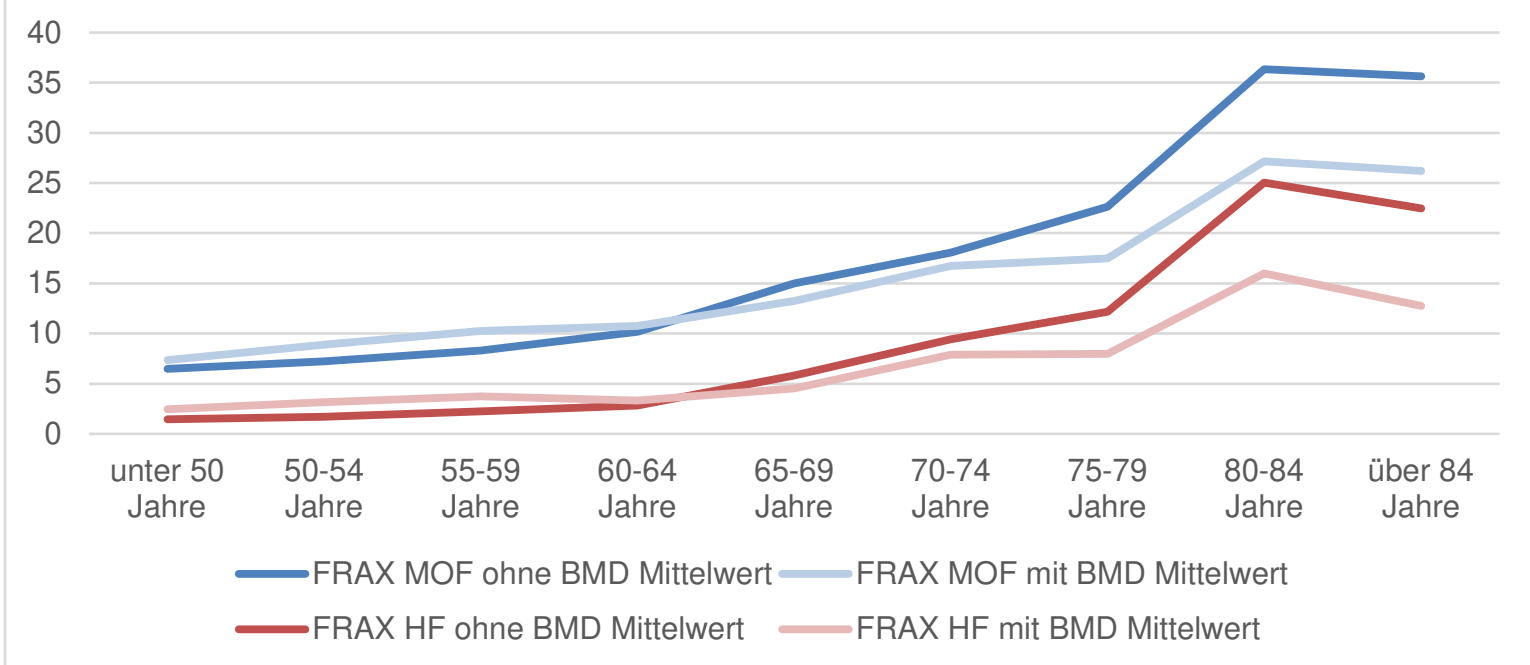

Abbildung 14: FRAX-Mittelwerte aufgetragen gegen Altersgruppen

Bei den Patientinnen unter 64 Jahren lag der FRAX-Mittelwert ohne BMD innerhalb der Altersgruppen unterhalb des Wertes mit BMD. Ab der Altersgruppe von 65 Jahren überstiegen die durchschnittlichen Mittelwerte ohne BMD die mit einbezogener BMD. Ein besonders deutlicher Sprung der Mittelwerte zeigte sich zwischen der Gruppe der 75- bis 79-Jährigen und den 80- bis 84-jährigen Patientinnen. Er erwies sich auf dem Niveau $p<0,01$ als signifikant. Der sichtbare Abfall der durchschnittlichen Mittelwerte bei den über 84-jährigen Patientinnen wurde als sich nicht signifikant von der Gruppe der 80- bis 84-Jährigen unterscheidend getestet. Das Frakturrisiko laut FRAX fällt also nicht signifikant ab, steigt aber auch nicht, wie zu erwarten wäre weiter an, sondern bleibt gleich. 


\subsubsection{Risikofaktoren in FRAX}

Die auf der FRAX-Eingabeseite für den FRAX-Rechner benötigten Risikofaktoren wurden im Kollektiv hinsichtlich der Häufigkeitsverteilung untersucht.

\subsubsection{Vorausgegangene Fraktur}

Mehr als die Hälfte der Patientinnen hatten bei Erstkontakt bereits eine für den FRAX-Rechner relevante Fraktur erlitten. Als FRAX-relevant gilt jede im Erwachsenenalter aufgetretenen Fraktur ohne oder mit nicht adäquatem Trauma. Eine genaue Angabe der Anzahl der Frakturen oder Differenzierung zwischen den einzelnen Lokalisationen wird im FRAX-Rechner nicht vorgenommen.

\section{Vorausgegangene Fraktur}

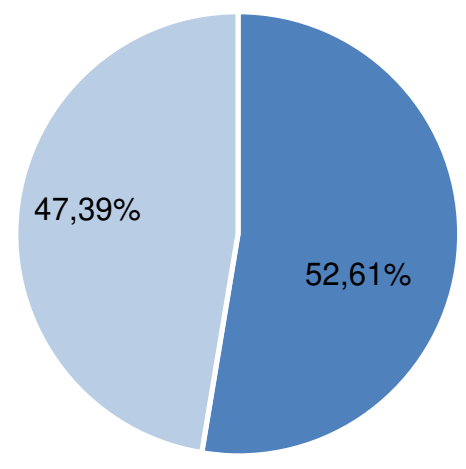

- Fraktur "ja" - Fraktur "nein"

Abbildung 15: Häufigkeitsverteilung „Vorausgegangene Fraktur ja/nein“

\subsection{Frakturtypen}

Zur ergänzenden Differenzierung zeigt Abbildung 16 die Verteilung der verschiedenen Frakturtypen innerhalb des Patientenkollektivs. Niedrigtraumatische Wirbelkörperfrakturen traten demnach bei $27 \%$ aller Patientinnen auf, wohingegen niedrigtraumatische multiple periphere Frakturen wie in der DVO-Leitlinie von 2014 berücksichtigt nur bei $4,5 \%$ aller Patientinnen aufgetreten waren. 


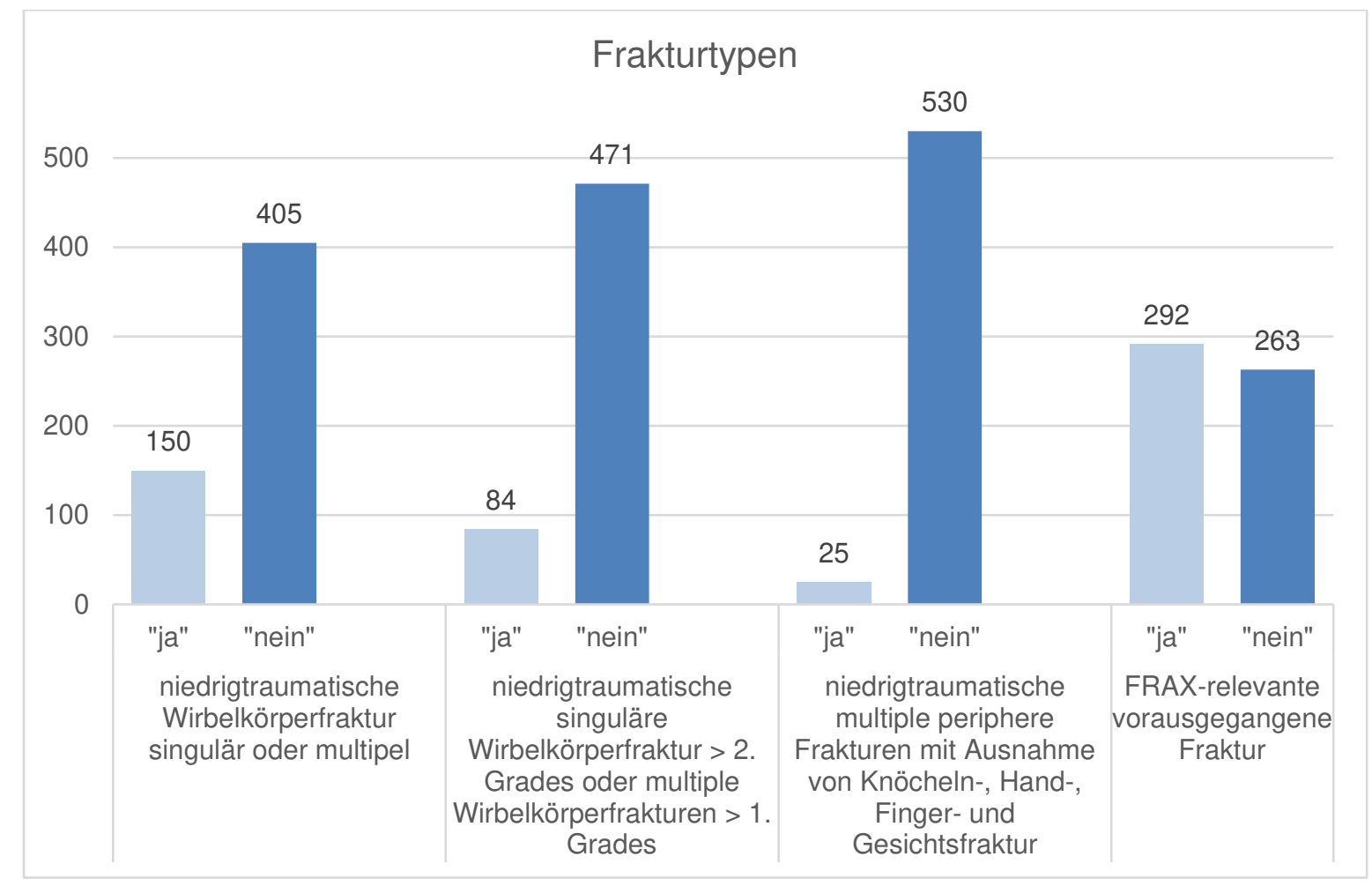

Abbildung 16: Verschiedene Frakturtypen. Die ersten drei Frakturkategorien werden laut DVOLeitlinien berücksichtigt. Ganz rechts zum Vergleich aufgetragen die Häufigkeitsverteilung der FRAXrelevanten Frakturen.

\subsubsection{Hüftfraktur eines Elternteils}

Hüftfrakturen bei mindestens einem Elternteil waren im vorliegenden Frauenkollektiv bei $13,9 \%$ der Patientinnen aufgetreten. Das entspricht einer absoluten Anzahl von 77 Patientinnen.

\section{Hüftfraktur eines Elternteils}

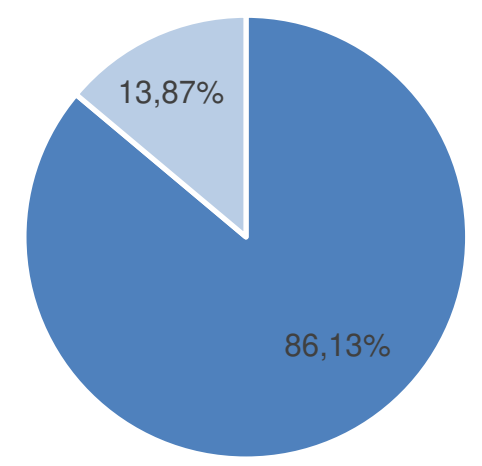

- Hüftfraktur Elternteil "nein" — Hüftfraktur Elternteil "ja"

Abbildung 17: Häufigkeitsverteilung „Hüftfraktur eines Elternteils ja/nein“ 


\subsubsection{Gegenwärtiges Rauchen}

Von den 555 Patientinnen ergab sich, dass 464 Patientinnen zum Aufnahmezeitung Nichtraucher waren. 16,4\% gaben an zum Aufnahmezeitpunkt zu rauchen. Das entsprach einer absoluten Anzahl von 91 Patientinnen.

\section{Gegenwärtiges Rauchen}

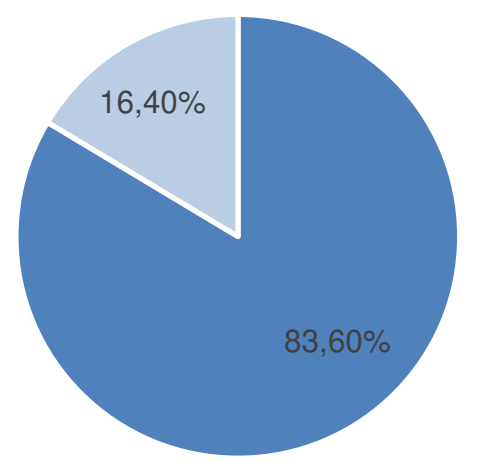

- Gegenwärtiges Rauchen "nein"

- Gegenwärtiges Rauchen "ja"

Abbildung 18: Häufigkeitsverteilung „Gegenwärtiges Rauchen ja/nein“

\subsubsection{Glukokortikoide}

Im betrachteten Kollektiv gaben 93 Patientinnen (16,76\%) an, zum Zeitpunkt der Aufnahme bzw. im Zeitraum von bis zu drei Monaten vor- bzw. nachher Glukokortikoide einzunehmen. Von diesen 93 Patientinnen erhielten 13 eine Dosierung von über 7,5 mg pro Tag Prednisolon-äquivalent. Die restlichen 80 Patientinnen nahmen zwar ebenfalls regelmäßig Glukokortikoide ein, allerdings in einer täglichen Dosierung unterhalb der 7,5 mg Grenze. Für die Eingabe in den FRAX-Rechner ist die Einnahme ab einer Dosis von $5 \mathrm{mg}$ relevant. 


\section{Glukokortikoideinnahme}

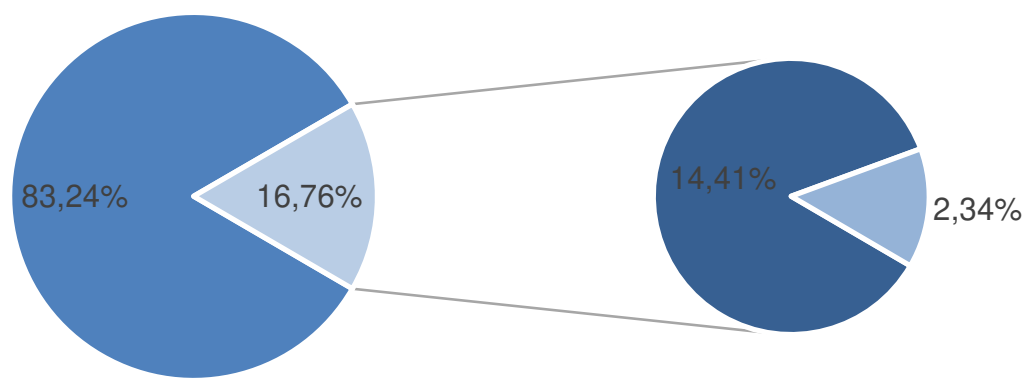

- Glukokortikoideinnahme "nein"

- Glukokortikoideinnahme "ja" > 5 mg < 7,5 mg/d Prednisolon-äquivalent

- Glukokortikoideinnahme "ja" > 7,5 mg/d Prednisolon-äquivalent

Abbildung 19: Häufigkeitsverteilung „Glukokortikoide ja/nein“ Links: Verteilung Glukortikoideinnahme ,ja“ (16,76 \%) vs. „,nein“ (83,24 \%). Rechts: Aufteilung des Anteils „Glukortikoideinnahme ,ja“ $(16,76 \%)$ in „,5-7,5 mg“ (14,41 \%) und „> 7,5 mg“ (2,34 \%).

\subsubsection{Rheumatoide Arthritis}

39 Patientinnen, entsprechend einem prozentualen Anteil von 7,03\% litten unter einer bei Aufnahme bereits diagnostizierten Rheumatoiden Arthritis.

\section{Rheumatoide Arthritis}

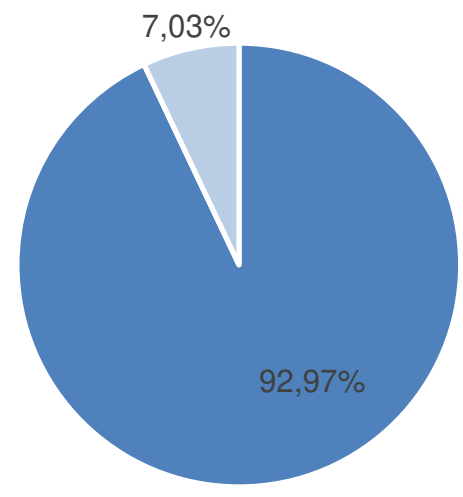

• Rheumatoide Arthritis "nein" — Rheumatoide Arthritis "ja"

Abbildung 20: Häufigkeitsverteilung „Rheumatoide Arthritis ja/nein“

\subsubsection{Sekundäre Osteoporose}

Eine Gesamtanzahl von 126 Patientinnen erfüllte die von FRAX geforderten Kriterien für eine sekundäre Osteoporose. Das entsprach einem prozentualen Anteil von $22,7 \%$. Dabei ist besonders hervorzuheben, dass von diesen 126 Patientinnen mit sekundärer Osteoporose 120 Patientinnen $(21,6 \%)$ als sekundär verschlüsselt wurden, da sie angegeben hatten, ihre Menopause hätte bereits vor 
dem 45. Lebensjahr begonnen. Das entspricht einem Anteil an den sekundären Osteoporosen von 95,23\%, unabhängig davon, ob sie eventuell ein zusätzliches zweites Kriterium für eine sekundäre Osteoporose laut FRAX erfüllt hätten.

\section{Sekundäre Osteoporose und Ursachen}

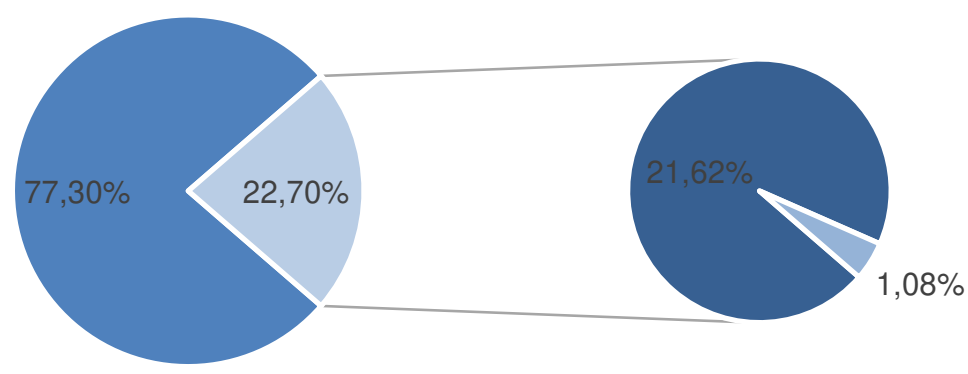

- sekundäre Osteoporose "nein"

- sekundäre Osteoporose "ja" "Menopause < 45-jährig"

- sekundäre Osteoporose "ja" "andere"

Abbildung 21: Häufigkeitsverteilung „sekundäre Osteoporose ja/nein“

Von den 293 Patientinnen mit 10-Jahres-Frakturrisiko über $30 \%$ hatten 226 (77,1 \%) eine primäre Osteoporose, 67 (22,9\%) hatten eine sekundäre Osteoporose laut FRAX-Kriterien. Von den nur 22 Patientinnen mit DVO-Frakturrisiko unter $20 \%$ hatten acht Patientinnen (36,4\%) eine sekundäre Osteoporoseursache.

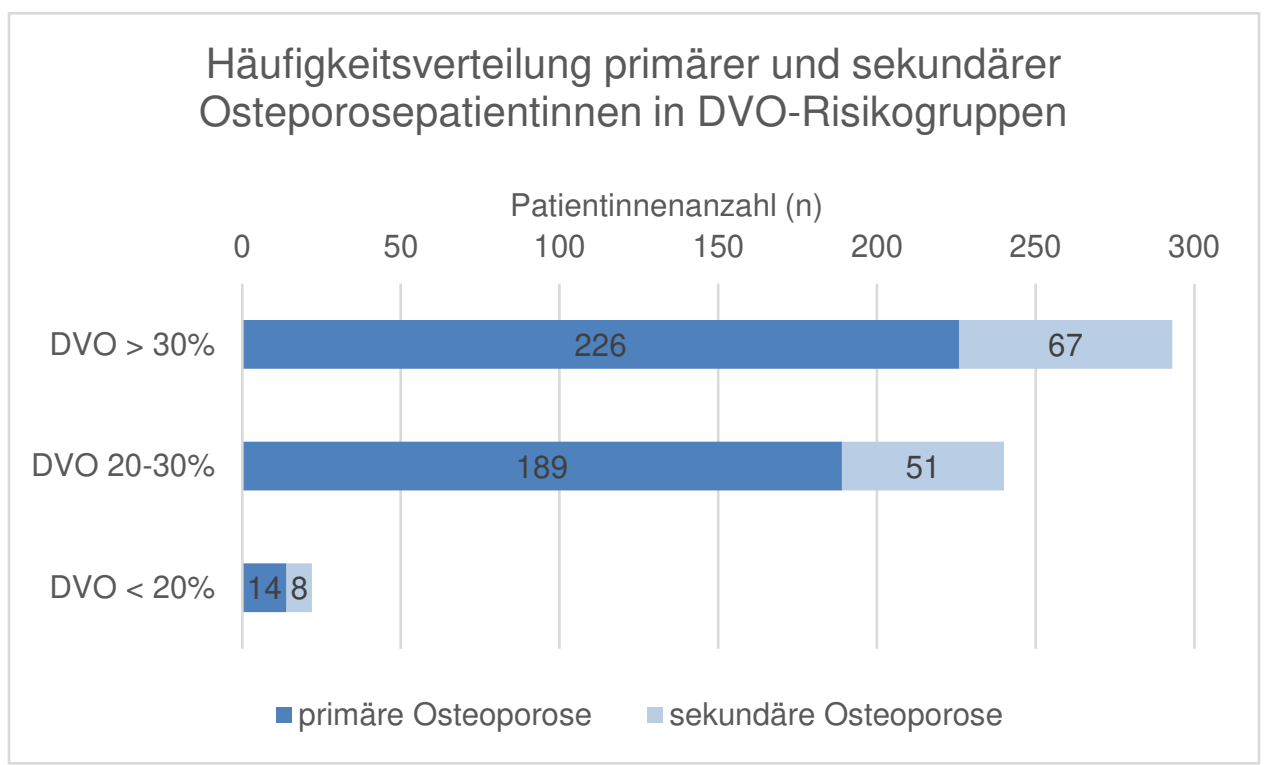

Abbildung 22: Häufgkeitsverteilung primärer und sekundärer Osteoporosepatientinnen in DVO10-Jahres-Frakturrisikogruppen (in absoluten Zahlen) 


\subsubsection{Alkoholkonsum}

Es gaben 48 Patientinnen, entsprechend $8,65 \%$ des Kollektivs, an, regelmäßig relevante Mengen an Alkohol in einem FRAX-relevanten Ausmaß zu sich zu nehmen.

\section{Alkoholkonsum}

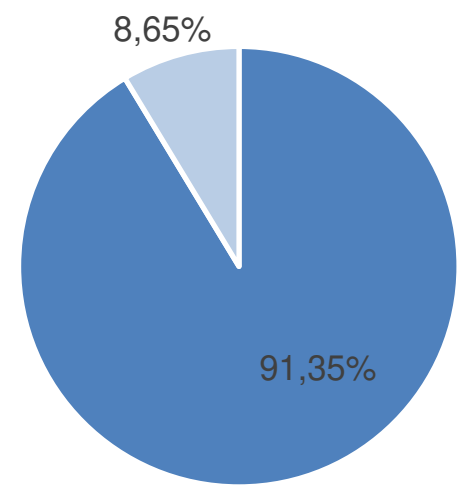

- Alkoholkonsum "nein" —Alkoholkonsum "ja"

Abbildung 23: Häufigkeitsverteilung „Alkoholkonsum ja/nein““

\subsubsection{Knochendichtemessung}

Zur Eingabe in den FRAX-Rechner wurden aus den vorhandenen Messungen am Femurhals der minimale T-Score beider Femurhälse ermittelt, falls beidseitige Messungen durchgeführt worden waren. Der durchschnittliche minimale T-Score der Femurhalsmessung lag im betrachteten Frauenkollektiv bei $-1,86( \pm 0,98)$. Minima und Maxima zeigt außerdem Tabelle 11.

Tabelle 11: Knochendichtemessung am Femurhals

\begin{tabular}{c|ccccc} 
& Mittelwert & SD & Maximum & Minimum & Median \\
\hline Minimaler T-Score & & & & & \\
Femurhalsmessung & $-1,86$ & 0,98 & 3,7 & $-4,5$ & $-1,9$
\end{tabular}

Laut Definition der WHO liegt eine Osteoporose bei einem T-Score kleiner oder gleich -2,5 vor. Diese Definition zu Grunde legend ergäbe sich eine mittels TScore des Femurhalses diagnostizierte Osteoporose bei 150 der 555 Patientinnen, entsprechend einem prozentualen Anteil von 27,03\%. Bei 304 der 555 Patientinnen läge eine Osteopenie mit einem minimalen T-Score zwischen -2,5 und -1 vor. Dies entsprach einem prozentualen Anteil von 54,77\%. Die restlichen 101 Patientinnen (18,2\%) hatten einen minimalen T-Score in der Femurhalsmessung der mindestens -1 betrug. 
Weiterhin ergab sich, dass der minimale T-Score am Femurhals bei Patientinnen mit Frakturereignis hochsignifikant niedriger war, als bei Patientinnen ohne vorausgegangene Fraktur $(p<0,01)$. Tabelle 12 zeigt die Mittelwerte und Standardabweichungen sowie Maxima und Minima innerhalb der Patientengruppe mit bzw. ohne vorausgegangene Fraktur.

Tabelle 12: Vergleich der durchschnittlichen T-Scores am Femurhals mit und ohne vorausgegangene Fraktur

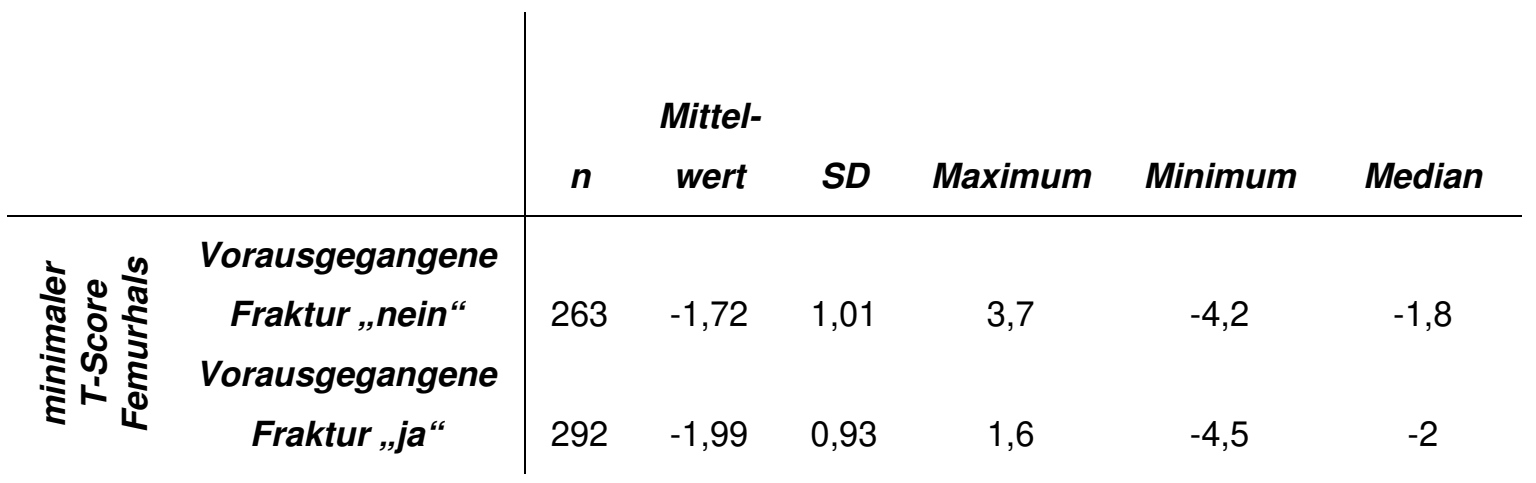

Wie erwartet zeigte sich ebenfalls ein hochsignifikanter Unterschied $(p<0,01)$ zwischen den T-Score Mittelwerten der DVO-Risikogruppen, mit einem durchschnittlichen T-Score von -2,21 in der Gruppe der Patientinnen mit einem 10Jahres-Frakturrisiko $>30 \%$.

Tabelle 13: Vergleich der durchschnittlichen T-Scores Femurhals innerhalb der DVO-Risikogruppen

\begin{tabular}{c|c|cccccc} 
& $\begin{array}{c}\text { DVO Frakturri- } \\
\text { siko }\end{array}$ & $\boldsymbol{n}$ & Mittelwert & SD & Maximum & Minimum & Median \\
\hline Minimaler & $<20 \%$ & 22 & $-0,24$ & 1,2 & 3,7 & $-1,7$ & $-0,6$ \\
T-Score & $20-30 \%$ & 240 & $-1,59$ & 0,87 & 1,8 & $-3,7$ & $-1,6$ \\
Femurhals & $>30 \%$ & 293 & $-2,21$ & 0,84 & 1,6 & $-4,5$ & $-2,2$
\end{tabular}

\subsection{Vergleich der Score-Risikowerte}

Im Anschluss folgte der Vergleich der verschiedenen Scores untereinander.

\subsubsection{Vergleich FRAX ohne BMD und DVO}

Zum Vergleich von FRAX- und DVO-Risikoscores wurden die Mittelwerte des FRAX-10-Jahresrisikos für eine MOF innerhalb der Risikogruppen nach DVOLeitlinie ermittelt. Die Mittelwerte unterschieden sich zwischen den einzelnen Risikogruppen hochsignifikant $(p<0,01)$. Der Mittelwert des FRAX-MOF-10-JahresFrakturrisikos in der Gruppe der laut DVO einem > 30 \%igen 10-JahresFrakturrisiko unterliegenden Patientinnen lag bei 18,2 $( \pm 11,4)$. 
Tabelle 14: DVO- und FRAX-Vergleich ohne Knochendichtemessung

\begin{tabular}{|c|c|c|c|c|c|c|c|}
\hline & \multicolumn{6}{|c|}{ FRAX MOF ohne BMD } \\
\hline & & $n$ & Mittelwert & $S D$ & Maximum & Minimum & Median \\
\hline \multirow{4}{*}{$\stackrel{0}{0}$} & $<20 \%$ & 22 & 8,47 & 5,86 & 24 & 2,5 & 6,45 \\
\hline & $20-30 \%$ & 240 & 9,8 & 7,13 & 52 & 2,1 & 8,25 \\
\hline & $>30 \%$ & 293 & 18,2 & 11,38 & 65 & 3,2 & 16 \\
\hline & Gesamt & 555 & 14,18 & 10,47 & 65 & 2,1 & 11 \\
\hline
\end{tabular}

Anzahl der Patientinnen innerhalb der drei DVO-Risikogruppen und deskriptive Statistik der FRAXRisikowerte für eine MOF ohne BMD in den jeweiligen Risikogruppen laut DVO.

Tabelle 15 zeigt die Übereinstimmung von DVO und FRAX MOF ohne BMD bei Gruppierung der FRAX-Risikowerte in Gruppen $<20 \%$, 20-30\% und $>30 \%$ parallelisiert zu der DVO-Risikoeinteilung. Von den 22 Patientinnen, die laut DVO ein Frakturrisiko $<20 \%$ zugeteilt bekamen, wurde 20 Patientinnen auch im FRAX ein Risiko unter $20 \%$ zugeordnet. Von den Patientinnen die laut DVO zwischen 20 und $30 \%$ 10-Jahres-Frakturrisiko eingeteilt worden waren, hatten $92,5 \%$ ein FRAX MOF 10-Jahres-Frakturrisiko von unter $20 \%$. Nur 14,7\% (43) der Patientinnen die laut DVO über $30 \%$ kategorisiert worden waren, hatten auch im FRAXRechner ein Risiko über 30 \% für eine MOF zugeordnet bekommen.

Tabelle 15: Übereinstimmung der 10-Jahres-Frakturwahrscheinlichkeiten bei Gruppierung der FRAXWerte ohne BMD

\begin{tabular}{|c|c|c|c|c|c|}
\hline & & \multirow[b]{2}{*}{$n$} & \multicolumn{3}{|c|}{ FRAX MOF ohne BMD } \\
\hline & & & $<20 \%$ & $20-30 \%$ & $>30 \%$ \\
\hline \multirow{4}{*}{$\stackrel{\rho}{a}$} & $<20 \%$ & 22 & $20(90,9 \%)$ & $2(9,1 \%)$ & $0(0 \%)$ \\
\hline & $20-30 \%$ & 240 & $222(92,5 \%)$ & $11(4,6 \%)$ & $7(2,9 \%)$ \\
\hline & $>30 \%$ & 293 & $188(64,2 \%)$ & $62(21,1 \%)$ & $43(14,7 \%)$ \\
\hline & Gesamtanzé & 555 & $430(77,5 \%)$ & $75(13,5 \%)$ & $50(9 \%)$ \\
\hline
\end{tabular}

\subsubsection{Vergleich FRAX mit BMD und DVO}

Auch die FRAX-Mittelwerte für eine MOF mit BMD innerhalb der DVORisikogruppen wurden bestimmt. Innerhalb der DVO-Risikogruppe $>30 \%$ ergab sich ein mittlerer Wert für eine MOF mit BMD von 17,4 $( \pm 10,8)$. Die Mittelwerte unterscheiden sich zwischen den einzelnen DVO-Risikogruppen hochsignifikant $(p<0,01)$. 
Tabelle 16: DVO- und FRAX-Vergleich bei eingeschlossener Knochendichtemessung

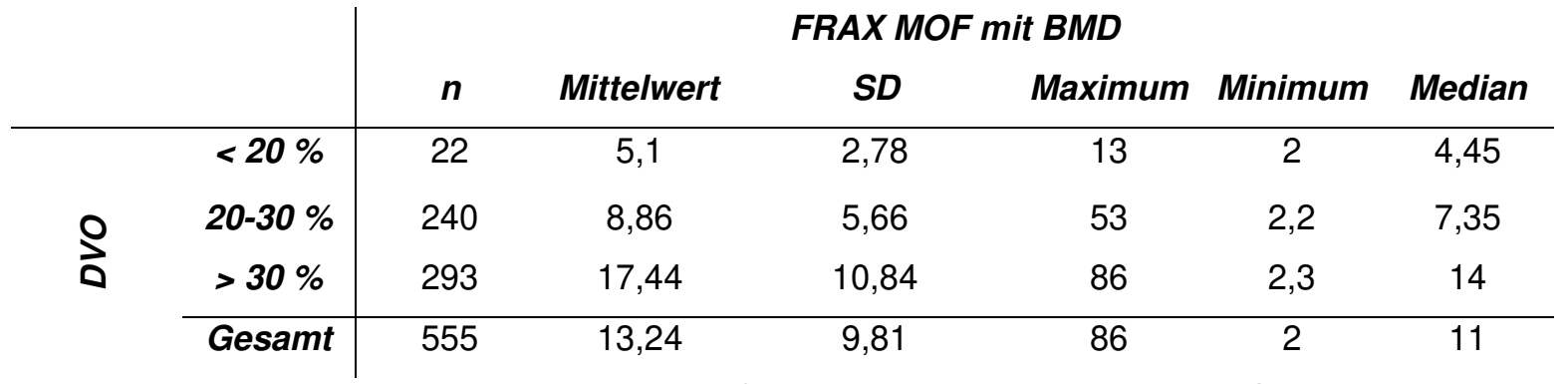

Anzahl der Patientinnen innerhalb der drei DVO-Risikogruppen und deskriptive Statistik der FRAXRisikowerte für eine MOF mit BMD innerhalb der jeweiligen Risikogruppe.

Bei einbezogener Knochendichtemessung wurden $100 \%$ der Patientinnen die laut DVO ein Risiko $<20 \%$ hatten ebenfalls im FRAX-Rechner ein unter $20 \%$ liegendes Frakturrisiko zugeteilt. Nur noch 12,97\% der Patientinnen der höchsten Risikogruppe $>30 \%$ laut DVO jedoch erhielten auch im FRAX Rechner ein 10Jahres-Frakturrisiko $>30 \%$.

Tabelle 17: Übereinstimmung der 10-Jahres-Frakturwahrscheinlichkeiten bei Gruppierung der FRAXWerte mit BMD

\begin{tabular}{ccc|ccc} 
& & & \multicolumn{3}{c}{ FRAX MOF mit BMD } \\
& & $\boldsymbol{n}$ & $<\mathbf{2 0} \%$ & $\mathbf{2 0 - 3 0 \%}$ & $>\mathbf{3 0} \%$ \\
\hline \multirow{3}{*}{$\mathrm{O}$} & $\mathbf{2}$ & 22 & $22(100 \%)$ & 0 & 0 \\
& $\mathbf{2 0 - 3 0 \%}$ & 240 & $230(95,8 \%)$ & $8(3,3 \%)$ & $2(0,9 \%)$ \\
& $>\mathbf{3 0} \%$ & 293 & $196(66,9 \%)$ & $61(20,8)$ & $36(12,3 \%)$ \\
\cline { 2 - 6 } & Gesamtanzahl & 555 & $448(80,7 \%)$ & $69(12,4 \%)$ & $38(0,5 \%)$
\end{tabular}

\subsubsection{Vergleich DVO-Risikowerte mit DVO+}

Zum Vergleich der DVO-Risikowerte mit den Risikowerten des Risikomodells DVO+ wurden letztere mit zehn multipliziert, um eine näherungsweise Vergleichbarkeit zu erzeugen. Von den 293 Patientinnen die laut DVO > 30 \% 10-JahresFrakturrisiko klassifiziert wurden, hatten 140 Patientinnen, entsprechend 47,8\% auch laut DVO+ ein 10-Jahres-Frakturrisiko $>30 \%$. Große Übereinstimmung fand sich in der Gruppe der laut DVO unter $<20 \%$ klassifizierten Patientinnen. 86,4 \% der Patientinnen, die laut DVO in diese Gruppe eingeordnet worden waren, hatten auch im DVO+ ein unter $20 \%$ liegendes 10-Jahres-Frakturrisiko erhalten. 
Tabelle 18: Übereinstimmung der 10-Jahres-Frakturwahrscheinlichkeiten bei Gruppierung der DVO+Werte hochgerechnet auf zehn Jahre

\begin{tabular}{ccc|ccc} 
& & & \multicolumn{3}{|c}{ DVO+ } \\
& & $\boldsymbol{n}$ & $<\mathbf{2 0} \%$ & $\mathbf{2 0 - 3 0} \%$ & $>\mathbf{3 0} \%$ \\
\hline \multirow{3}{*}{$\mathbf{0}$} & $\mathbf{2 0} \%$ & 22 & $19(86,4 \%)$ & 0 & $3(13,6)$ \\
& $\mathbf{2 0 - 3 0 \%}$ & 240 & $205(85,4 \%)$ & $16(6,7 \%)$ & $19(7,9 \%)$ \\
& $>\mathbf{3 0} \%$ & 293 & $118(40,3 \%)$ & $35(11,9 \%)$ & $140(47,8 \%)$ \\
\cline { 2 - 6 } & Gesamtanzahl & 555 & $342(61,6 \%)$ & $51(9,2 \%)$ & $162(29,2 \%)$
\end{tabular}

\subsubsection{Vergleich FRAX- und DVO+- 1-Jahres-Risikowerte}

Zur Vergleichbarkeit der Risikoeinschätzungen von FRAX und DVO+ wurde das 1Jahres-Risiko laut FRAX ermittelt, indem die Werte des FRAX Rechners durch zehn geteilt wurden, um näherungsweise 1-Jahres-Frakturrisikowerte zu erzeugen. Graphisch dargestellt sind die wesentlich niedrigeren Verläufe der Kurve der Mittelwerte der FRAX-1-Jahres-Risikos eindrücklich erkennbar. Sichtbar wird auBerdem, dass die Differenz der 1-Jahres-Mittelwerte zwischen DVO und FRAX mit höherer Altersgruppe zunimmt. Besonders stark ist der Anstieg ab den über 65jährigen ausgeprägt. Ab dieser Altersgruppe ist laut DVO+-Risikotabelle ein Risiko von größer als zehn Prozent 1-Jahres-Frakturwahrscheinlichkeit möglich und somit der „wahre“ Anstieg der Mittelwerte unter Einbeziehung von Werten über zehn Prozent vermutlich noch höher einzuschätzen. Die absoluten DVO+-Risikowerte unterschieden sich über die Altersgruppen hochsignifikant $(p<0,01)$. Interessant ist auch der Verlauf der Kurve des relativen Frakturrisikos im Vergleich zum absoluten Risiko. Das relative Risiko unterschied sich über die verschiedenen Altersgruppen signifikant $(p<0,05)$. Es ergibt sich aus der Multiplikation der beiden höchsten Risikofaktorwerte ohne dass das Alter an dieser Stelle einfließt. In den niedrigen Altersgruppen liegen die relativen Risikowerte noch über den absoluten Werten. Der Schnittpunkt der beiden Verlaufskurven liegt zwischen den Altersgruppen der 70- bis 74-jährigen und der 75- bis 79-jährigen Patientinnen. Ab hier korrigierte das einfließende Alter das absolute Risiko eher nach oben als nach unten. Weiterhin erfolgte ab hier eine leichte Annäherung der Kurven des absoluten und relativen DVO+-1-Jahres-Risikos. 


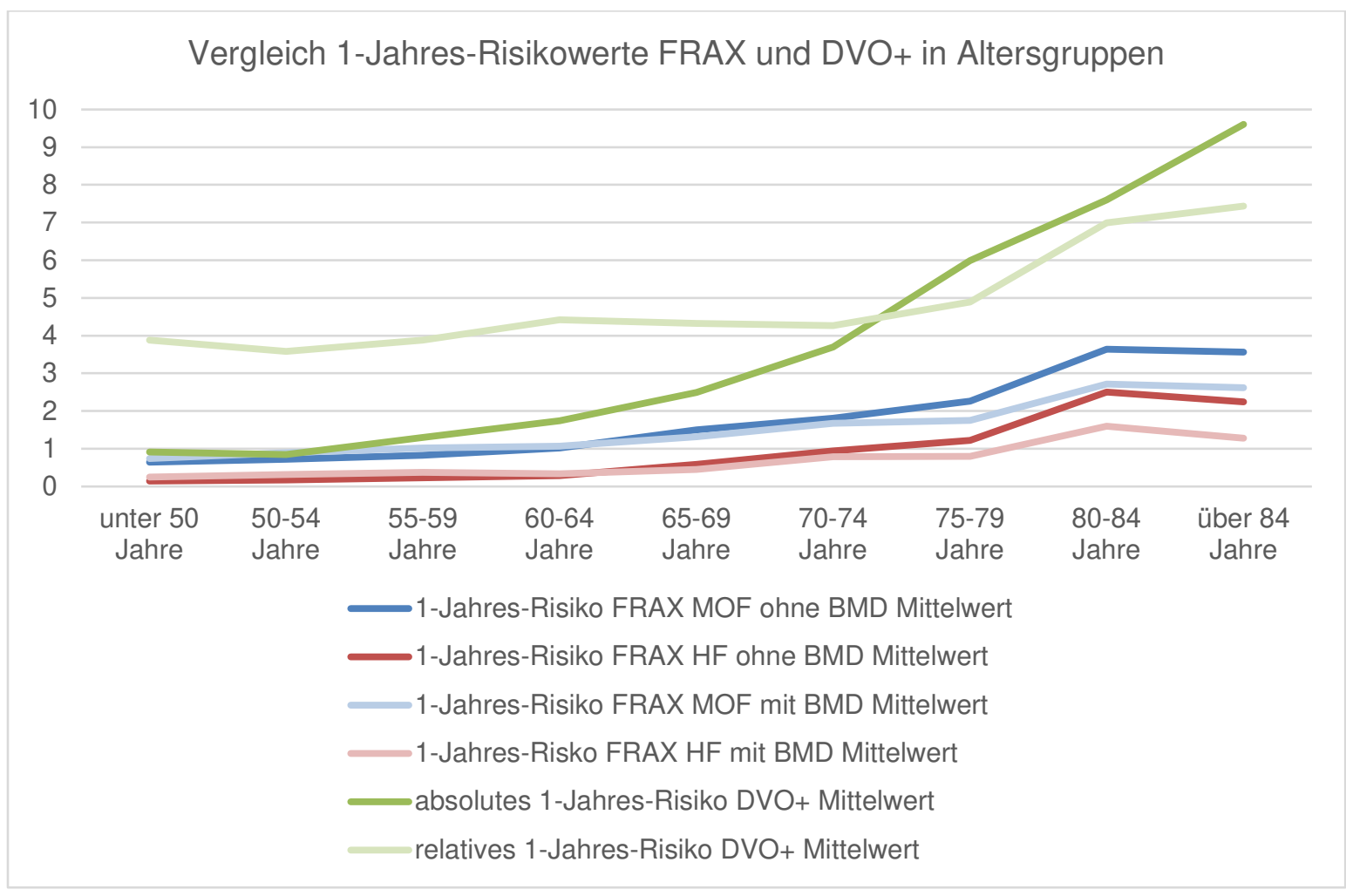

Abbildung 24: Vergleich der 1-Jahres-Frakturrisikowerte von FRAX und DVO+ innerhalb der Altersgruppen des DVO+-Risikomodells

Die Scores DVO+ und FRAX (1-Jahres-Frakturwahrscheinlichkeiten) sowie die FRAX-1-Jahres-Frakturwerte mit und ohne BMD bzw. MOF und HF unterscheiden sich voneinander hochsignifikant.

\subsubsection{Korrelation der gruppierten 10-Jahres-Frakturrisikowerte der Scores}

Bei Einteilung aller Scores in drei 10-Jahres-Frakturrisikogruppen > $20 \%, 20$ $30 \%,>30 \%$ zeigte sich eine hochsignifikante Korrelation untereinander zwischen allen Scores. Die entsprechenden Korrelationskoeffizienten zeigt Tabelle 19.

Tabelle 19: Korrelation der gruppierten Scores DVO, FRAX MOF ohne und mit BMD und DVO+

\begin{tabular}{c|c|c|c|c|}
$\begin{array}{c}\text { Korrelation gruppierte } \\
\text { Scores }\end{array}$ & DVO & FRAX MOF ohne BMD & FRAX MOF mit BMD & DVO+ \\
\hline DVO & 1 & & & \\
\hline FRAX MOF ohne BMD & $0,316^{\star *}$ & 1 & 1 & \\
\hline FRAX MOF mit BMD & $0,355^{\star *}$ & $0,523^{\star *}$ & $0,332^{\star *}$ & 1
\end{tabular}




\subsection{Vergleich der Therapieindikationen der Scores}

\subsubsection{Therapieindikation laut FRAX}

Publizierte internationale Therapiegrenzen von $\geq 20 \%$ MOF 10-JahresFrakturrisiko und $\geq 3 \%$ HF 10-Jahres-Frakturrisiko zugrunde legend wurde die Anzahl der Patientinnen, die dieser Empfehlung folgend Therapieindikation hätten, eruiert. Es zeigte sich, dass 56,04 \% der Patientinnen ohne eingeschlossene Knochendichtemessung und 49,01\% mit eingeschlossener Knochendichtemessung ein MOF-Risiko $\geq 20 \%$ und/oder ein HF-Risiko $\geq 3 \%$ hatten und damit die Kriterien zur Therapieindikation erfüllten. Dabei wird sichtbar, dass der größere Anteil der Patientinnen bei Betrachtung des Risikos HF $\geq 3 \%$ im Vergleich zur MOFTherapiegrenze als therapieindiziert eingeschätzt würde.

Tabelle 20: Therapieindikation laut FRAX mit publizierter internationaler $\geq 20 \%$ MOF und $\geq 3 \%$ HF 10 Jahres-Frakturrisiko-Therapiegrenze

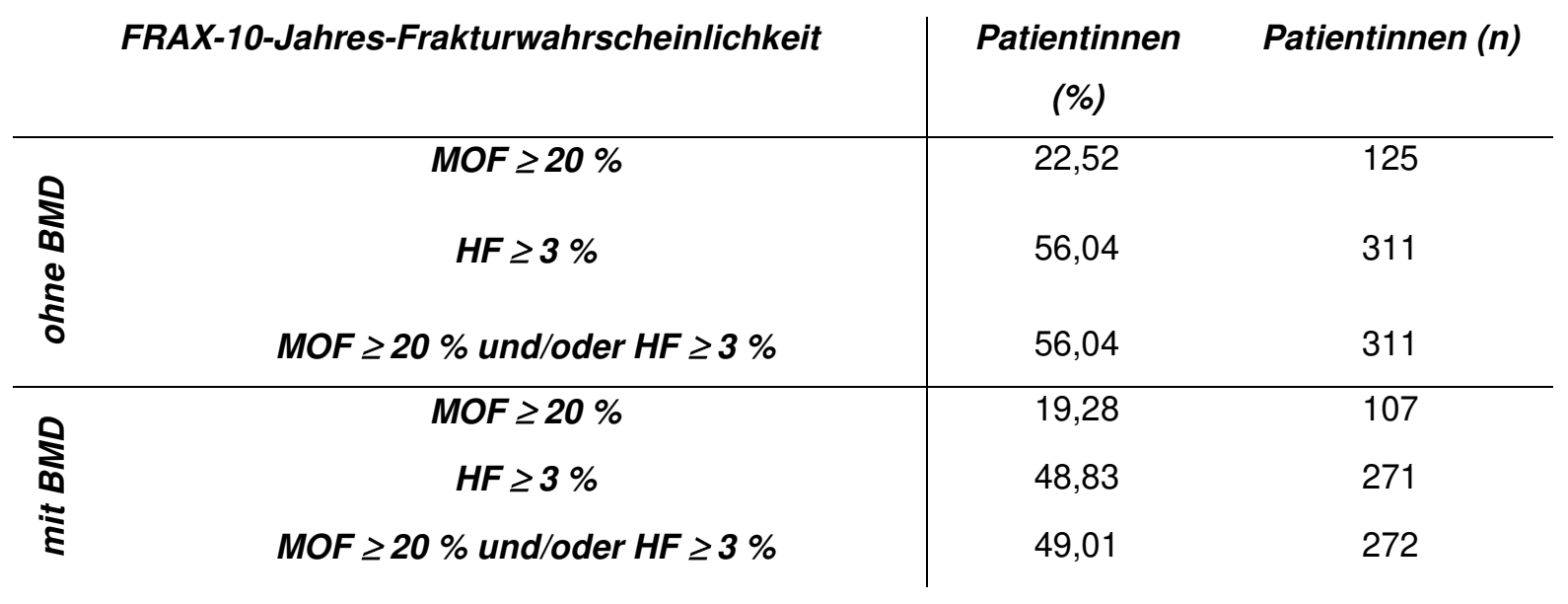

Die Empfehlung der DVO-Leitlinien 2014 zugrunde legend, wurde ebenfalls eruiert, wie viele Patientinnen laut FRAX therapiert werden würden, sofern die Therapiegrenzen mit $\geq 14 \%$ für eine MOF und/oder $\geq 3 \%$ für eine $\mathrm{HF}$ festgelegt würden. Es ergab sich ein prozentualer Anteil von die Therapiegrenzen überschreitenden Patientinnen von 56,76\% ohne Knochendichtemessung und 50,45\% mit Knochendichtemessung. 
Tabelle 21: Therapieindikation laut FRAX mit publizierter $\geq 14 \%$ MOF und $\geq 3 \%$ HF 10-JahresFrakturrisiko-Therapiegrenze

\begin{tabular}{|c|c|c|c|}
\hline \multicolumn{2}{|c|}{ FRAX-10-Jahres-Frakturwahrscheinlichkeit } & Patientinnen (\%) & Patientinnen ( $n$ ) \\
\hline \multirow{3}{*}{ 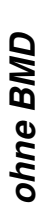 } & MOF $\geq 14 \%$ & 40,18 & 223 \\
\hline & $H F \geq 3 \%$ & 56,04 & 311 \\
\hline & MOF $\geq 14 \%$ und/oder HF $\geq 3 \%$ & 56,76 & 315 \\
\hline \multirow{3}{*}{ 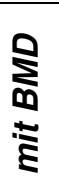 } & MOF $\geq 14 \%$ & 34,95 & 194 \\
\hline & $H F \geq 3 \%$ & 48,83 & 271 \\
\hline & MOF $\geq 14 \%$ und/oder $H F \geq 3 \%$ & 50,45 & 280 \\
\hline
\end{tabular}

Es ergab sich kein signifikanter Unterschied zwischen den therapierten Patientinnen laut FRAX mit und ohne BMD für eine zugrunde gelegte Therapiegrenze MOF 10-Jahresrisiko $\geq 20 \%$. Signifikante Unterschiede zwischen den Indikationen mit und ohne BMD ergaben sich zwischen den therapierten Patientinnen bei zugrunde gelegten Grenzen von MOF $\geq 14 \%$ sowie HF $\geq 3 \%$.

\subsubsection{FRAX-Therapieschwellen bezogen auf DVO-> $30 \%$-Anteil des Kollek- tivs}

Für das Kollektiv der 293 Patientinnen mit Therapieindikation laut DVO wurden die FRAX-Mittelwerte berechnet. Es ergab sich ein mittleres 10-Jahres-Risiko für eine MOF von $17,4 \%( \pm 10,8)$ mit und $18,2 \%( \pm 11,4)$ ohne eingeschlossene Knochendichtemessung. Für die Hüftfrakturen ergeben sich niedrigere durchschnittliche Werte von $8,1 \%( \pm 9,1)$ mit und $8,9 \%( \pm 9,5)$ ohne eingeschlossene Knochendichtemessung.

Tabelle 22: FRAX-Mittelwerte bezogen auf Patientinnen mit DVO 10-Jahres-Frakturrisiko $>30 \%$ ( $n=293)$

\begin{tabular}{cc|cc} 
DVO-10-Jahres-Frakturrisiko $>\mathbf{3 0} \%(\boldsymbol{n}=\mathbf{2 9 3})$ & ohne $\mathbf{B M D}$ & mit $\mathbf{B M D}$ \\
\hline \multirow{2}{*}{ FRAX-Risiko MOF } & Mittelwert & 18,2 & 17,4 \\
& Minimum & 3,2 & 2,3 \\
& Maximum & 65 & 86 \\
& SD & 11,4 & 10,8 \\
\hline \multirow{2}{*}{ FRAX-Risiko HF } & Mittelwert & 8,9 & 8,1 \\
& Minimum & 0,1 & 0 \\
& Maximum & 55 & 84 \\
& SD & 9,5 & 9,1
\end{tabular}

Bezogen auf die 293 Patientinnen mit DVO-Therapieindikation ergab sich, dass von diesen rund $54,3 \%$ bei eingeschlossener Knochendichtemessung und Thera- 
piegrenze von $\geq 14 \%$ für eine MOF therapiert werden würden. Bei Erhöhung der Therapieschwelle auf $\geq 20 \%$ Risiko für eine MOF mit eingeschlossener Knochendichtemessung würden nur noch gut ein Drittel der Patientinnen, die laut DVO therapiert werden würden, ebenfalls therapiert werden. Mehr Patientinnen mit einem 10-Jahres-Frakturrisiko über $30 \%$ würden therapiert werden, sofern man sich auf die Therapiegrenze von $\geq 3 \%$ Frakturrisiko für eine HF bezieht, nämlich $72,4 \%$ bei eingeschlossener Knochendichtemessung.

Es ergaben sich signifikante Unterschiede zwischen MOF $\geq 14 \%$ bzw. $\geq 20 \%$ und HF und den zusammengefassten Therapieschwellen zwischen den therapierten Patientenanteilen. Kein signifikanter Unterschied bestand zwischen den nach HF $\geq 3 \%$ therapierten Patientenanteilen und denen bei zusammengefassten Therapieschwellen weder mit noch ohne einbezogene Knochendichtemessung. Es ergibt sich kein signifikanter Unterschied der Bewertung mit oder ohne einbezogene DXA-Messung.

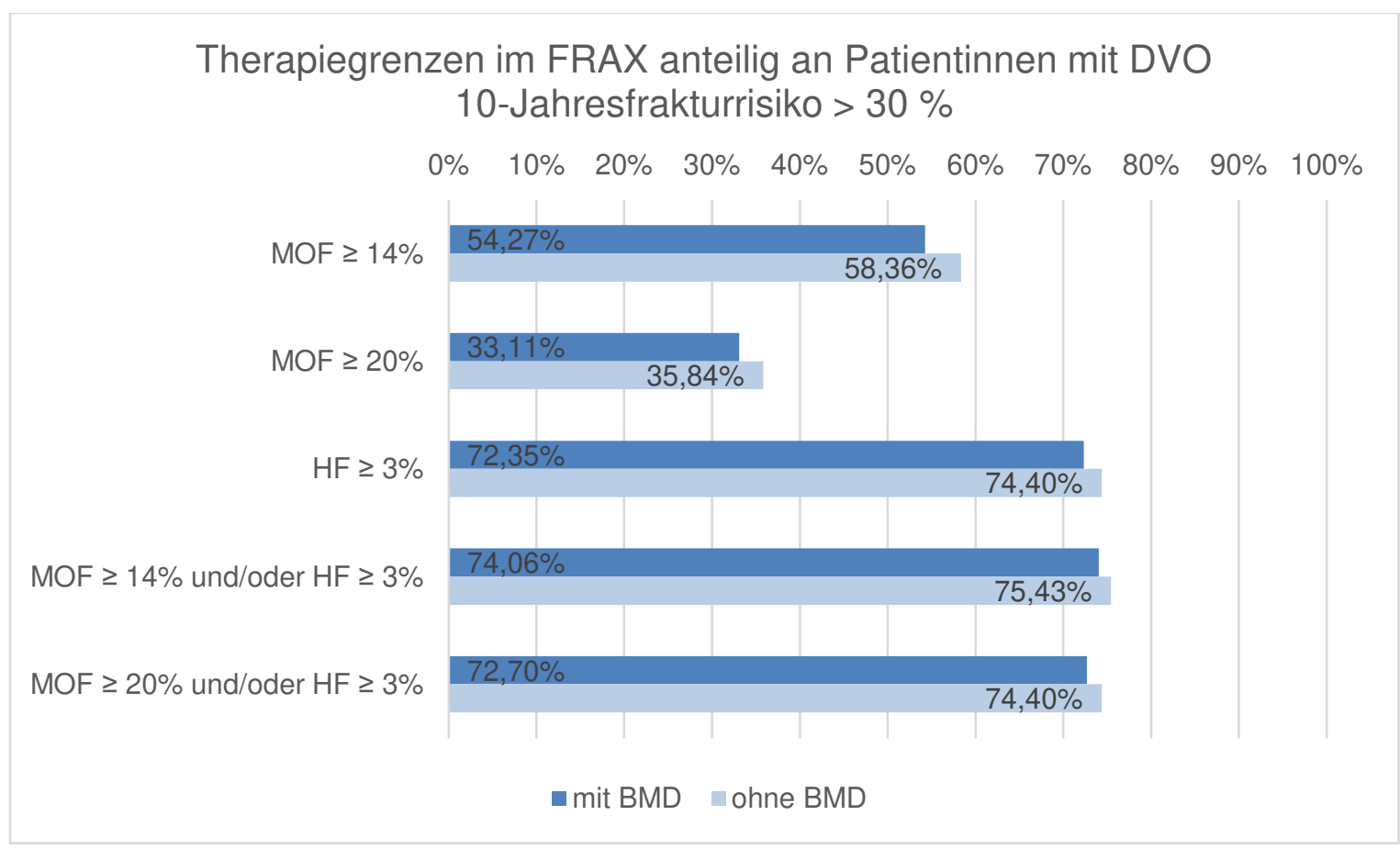

Abbildung 25: Therapiegrenzen im FRAX anteilig an Patientinnen mit DVO 10-Jahres-Frakturrisiko $>30 \%(n=293)$

\subsubsection{Therapieschwellen laut FRAX bezogen auf DVO 20-30\% Anteil des Kollektivs}

Analog wurden die FRAX-Mittelwerte auch für das Patientenkollektiv der Patientinnen berechnet, die laut DVO ein zwischen 20 und $30 \%$ liegendes Risiko für eine 10-Jahres-Fraktur hatten. Es ergab sich ein mittleres Risiko einer MOF von 
9,8\% $( \pm 7,1)$ ohne einbezogene Knochendichte und 8,9\% $( \pm 5,6)$ bei eingeflossenem Knochendichtemessungsergebnis. Für die Hüftfrakturwahrscheinlichkeit ergab sich ein durchschnittlicher Wert von 3,4\% $( \pm 4,7)$ ohne, bzw. 2,6 \% $( \pm 3,9)$ mit einbezogener Knochendichte. Auch in dieser Patientengruppe wird die 10Jahres-Frakturwahrscheinlichkeit laut FRAX bei einfließender Knochendichtemessung nach unten korrigiert.

Tabelle 23: FRAX-Mittelwerte bezogen auf Patientinnen mit DVO 10-Jahres-Frakturrisiko 20-30\% ( $n=240)$

\begin{tabular}{cc|cc} 
DVO-10-Jahres-Frakturrisiko 20-30 \% $(\boldsymbol{n}=\mathbf{2 4 0})$ & ohne BMD & mit BMD \\
\hline & Mittelwert & 9,8 & 8,9 \\
FRAX-Risiko MOF & Minimum & 2,1 & 2,2 \\
& Maximum & 52 & 53 \\
FRAX-Risiko HF & SD & 7,1 & 5,6 \\
\hline & Mittelwert & 3,4 & 2,6 \\
& Minimum & 0,1 & 0
\end{tabular}

Bezogen auf die 240 Patientinnen, denen laut DVO ein Risiko zwischen 20 und $30 \%$ 10-Jahres-Frakturwahrscheinlichkeit zugeordnet wurde, würden $14,58 \%$ laut FRAX bei Therapiegrenze MOF14 \% mit einbezogener Knochendichte therapiert werden. Lediglich 4,17\% erhielten Therapie bei einer Grenze bei MOF $20 \%$. Erneut wurde ein größerer Teil der Patientinnen als therapiepflichtig eingeschätzt, sofern man das Risiko für eine Hüftfraktur bei eingeschlossener Knochendichtemessung mit einbezieht ( $36,25 \%$ bzw. 36,67\%).

Signifikante Unterschiede ergeben sich zwischen den Therapieempfehlungen bezüglich eingeschlossener und nicht eingeschlossener Knochendichtemessung nur bei der Therapiegrenze MOF $\geq 14 \%$ nicht $(p=0,067)$. Jeweils signifikante Unterschiede gibt es bezüglich der Therapieempfehlungen zwischen MOF $\geq 20 \%$ mit und ohne BMD, sowie $H F \geq 3 \%$ mit und ohne BMD, sowie jeweils bei den zusammengefassten Therapieempfehlungsgrenzen mit und ohne BMD. Untereinander unterscheiden sich die Therapieempfehlungen jeweils signifikant. Ausgenommen hiervon sind jeweils die Therapieempfehlungen bei gewählte Grenze HF $\geq 3 \%$, die sich sowohl mit als auch ohne Knochendichtemessung nicht signifikant von den zusammengefassten Therapiegrenzen MOF $\geq 20 \%$ und HF $\geq 3 \%$ sowie MOF $\geq 14 \%$ und $\mathrm{HF} \geq 3 \%$ unterscheiden. 


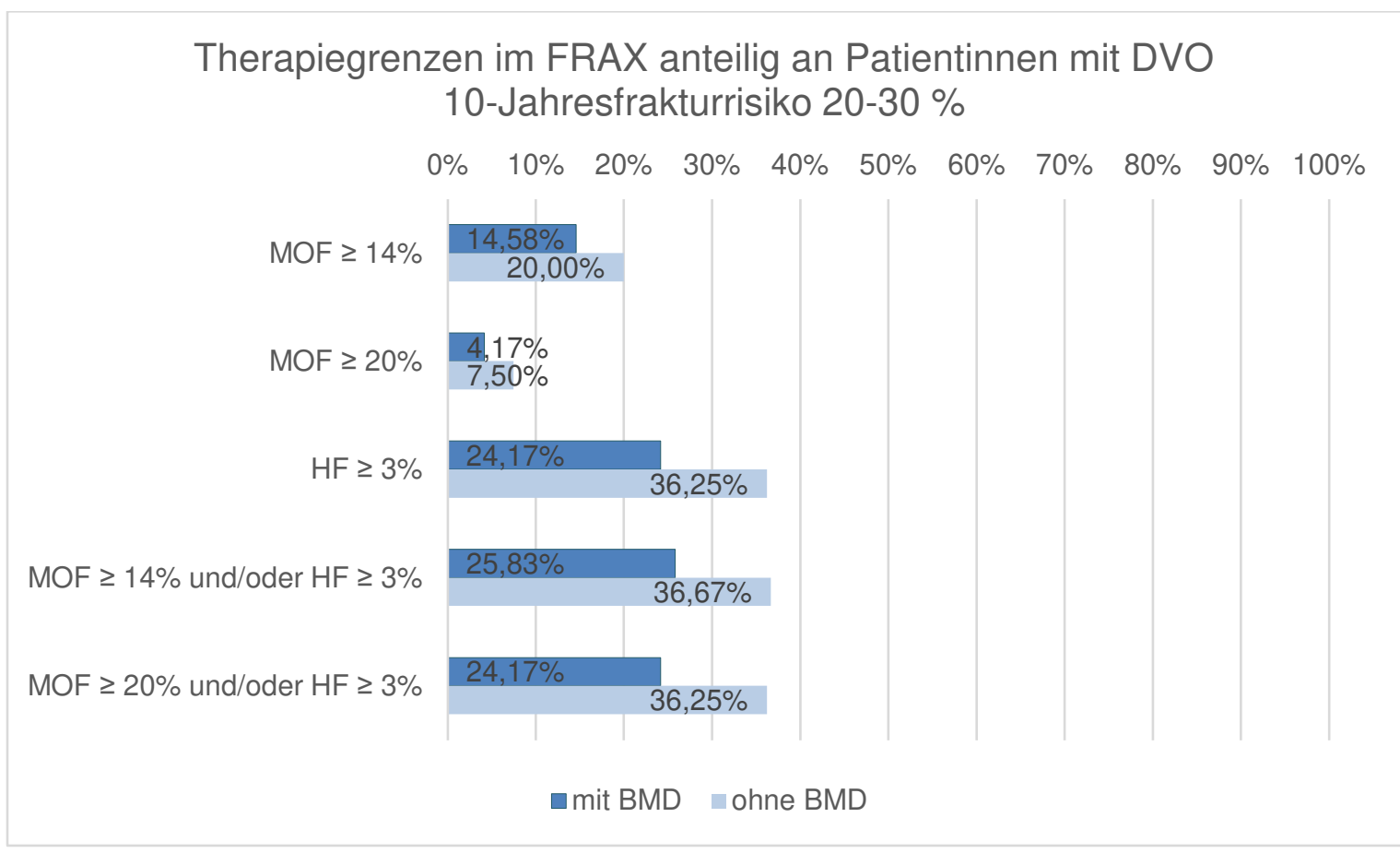

Abbildung 26: Therapiegrenzen im FRAX anteilig an Patientinnen mit DVO 10-Jahres-Frakturrisiko 20$30 \%(n=240)$

\subsubsection{Therapieindikation laut DVO+}

Therapieindikation laut DVO+ besteht bei > $6 \%$ 1-Jahres-Frakturrisiko oder > $3 \%$ 1-Jahres-Frakturrisiko bei gleichzeitig vorliegendem T-Score <-2. 129 der 555 Patientinnen $(23,24 \%)$ erfüllten diese Kriterien und hätten somit Therapieindikation laut DVO+. Von den 293 Patientinnen mit Therapieindikation laut DVO hatten 114 Patientinnen, entsprechend einem Anteil von 38,9\%, übereinstimmend auch eine Therapieindikation laut DVO+.

\subsubsection{Vergleich Therapieindikationen DVO, DVO+, FRAX}

Die meisten Patientinnen mit Therapieindikation werden detektiert, sofern man eine FRAX-Therapiegrenze von $\geq 3 \%$ FRAX-10-Jahres-Frakturrisiko für eine Hüftfraktur zugrunde legt $(56,04 \%)$ ohne die Knochendichtemessung mit einzubeziehen. Es würden so sogar noch mehr Patientinnen detektiert werden, als durch den DVO-Score $(52,79 \%)$. Die wenigsten Patientinnen wurden zur Therapieindikation identifiziert, sofern man eine Therapiegrenze von FRAX-10-Jahres-Frakturrisiko $\geq 20 \%$ für eine MOF mit BMD als Kriterium wählt.

Sichtbar wird weiterhin, dass laut DVO+-Modell weniger Patientinnen Therapieindikation hatten, als in den Risikoeinschätzungen nach DVO. Weniger Patientinnen hatten Therapieindikation bei eingeschlossener Knochendichtemessung in den FRAX-Rechner als ohne. 
Es ergibt sich kein signifikanter Unterschied hinsichtlich der Therapieempfehlungen zwischen DVO-Score und FRAX-Score bei zugrunde gelegter Grenze von $\geq 3 \%$ für eine Hüftfraktur sowohl mit als auch ohne eingeschlossener Knochendichtemessung.

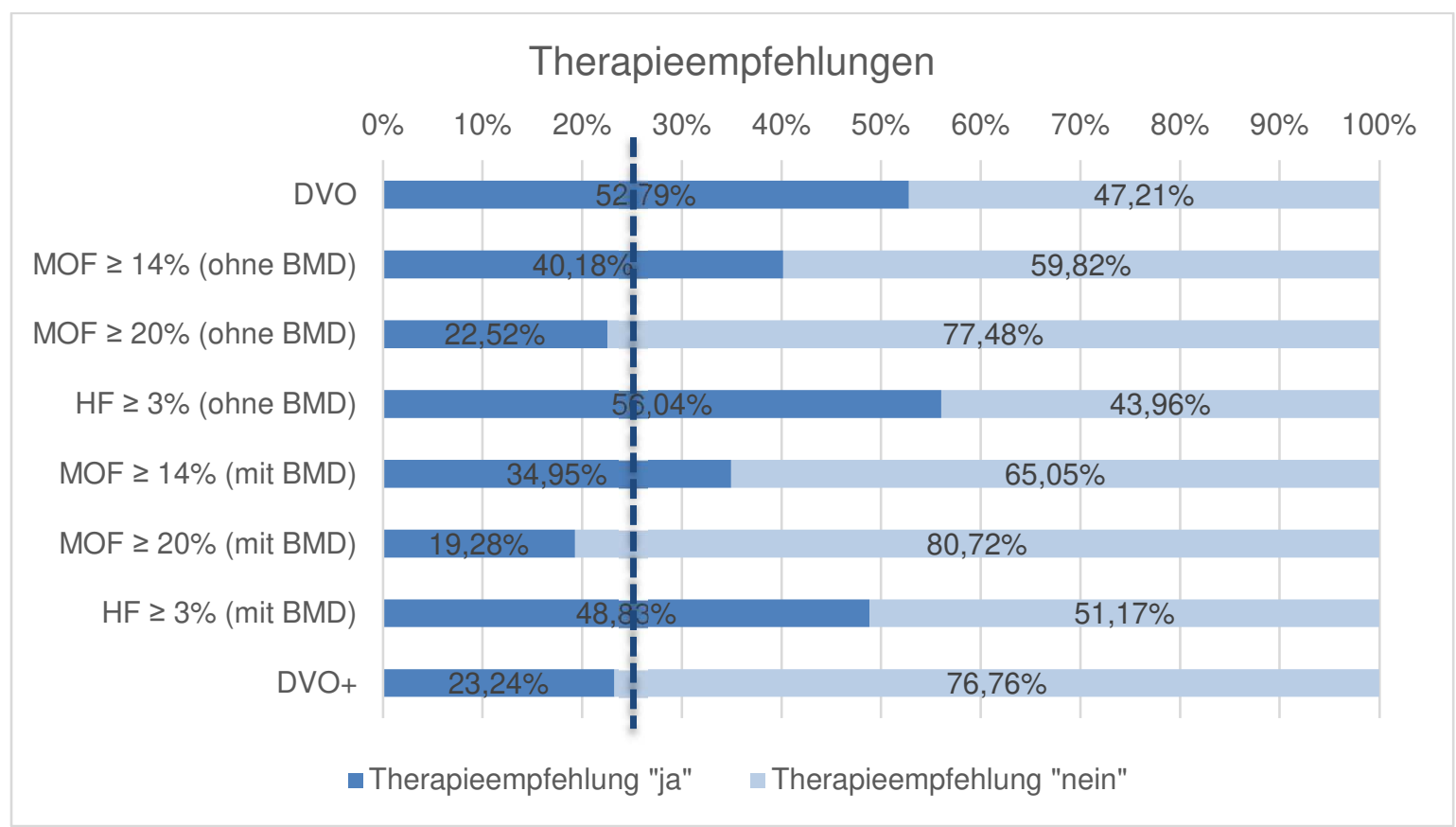

Abbildung 27: Therapieempfehlung ( $\mathrm{n}=555$ ) ,ja“ (mittelblau) und „,nein“ (hellblau) laut DVO, FRAX (gruppiert in Therapiegrenzen für MOF und HF), sowie DVO+. Die dunkelblau gestrichelte Linie zeichnet die Therapieempfehlung basierend auf einem T-Score $\leq-2,5$ ein.

Zur genaueren Differenzierung wurde nun für die jeweilige Therapiegrenze die Übereinstimmung ausgezählt und benannt, bei wie vielen Patientinnen jeweils laut DVO und FRAX, nur laut DVO bzw. nur laut FRAX Therapieindikation gestellt worden wäre. Die höchste prozentuale Übereinstimmung ergab sich bei der Therapiegrenze von HF $3 \%$ ohne eingeschlossene Knochendichtemessung $(39,28 \%)$. Weitere $16,76 \%$ wären bei dieser angelegten Therapiegrenze laut FRAX therapiert worden, nicht aber laut DVO als therapiewürdig eingeschätzt worden. Der DVO-Score erkannte 13,51\% Patientinnen, die laut FRAX bei Therapiegrenze HF $3 \%$ keine Therapieindikation hatten. Insgesamt wurden $69,54 \%$ der Patientinnen als therapiewürdig entweder laut DVO oder laut HF $3 \%$ ohne BMD Grenze identifiziert. Bei einbezogener Knochendichtemessung und Therapiegrenze HF $3 \%$ ergaben sich ähnlich hohe Übereinstimmungen zum DVO-Score $(38,2 \%)$. Nur 10,63\% allerdings erhielten hier zusätzlich Therapieindikation laut FRAX nicht aber laut DVO. 
Geringste Übereinstimmung zwischen FRAX und DVO herrschte bei gewählter Therapiegrenze von $20 \%$ MOF mit BMD. Hier wurden nur 17,48 \% übereinstimmend erkannt. Laut DVO-Score würde weitere $35,32 \%$ Patientinnen therapiert werden.

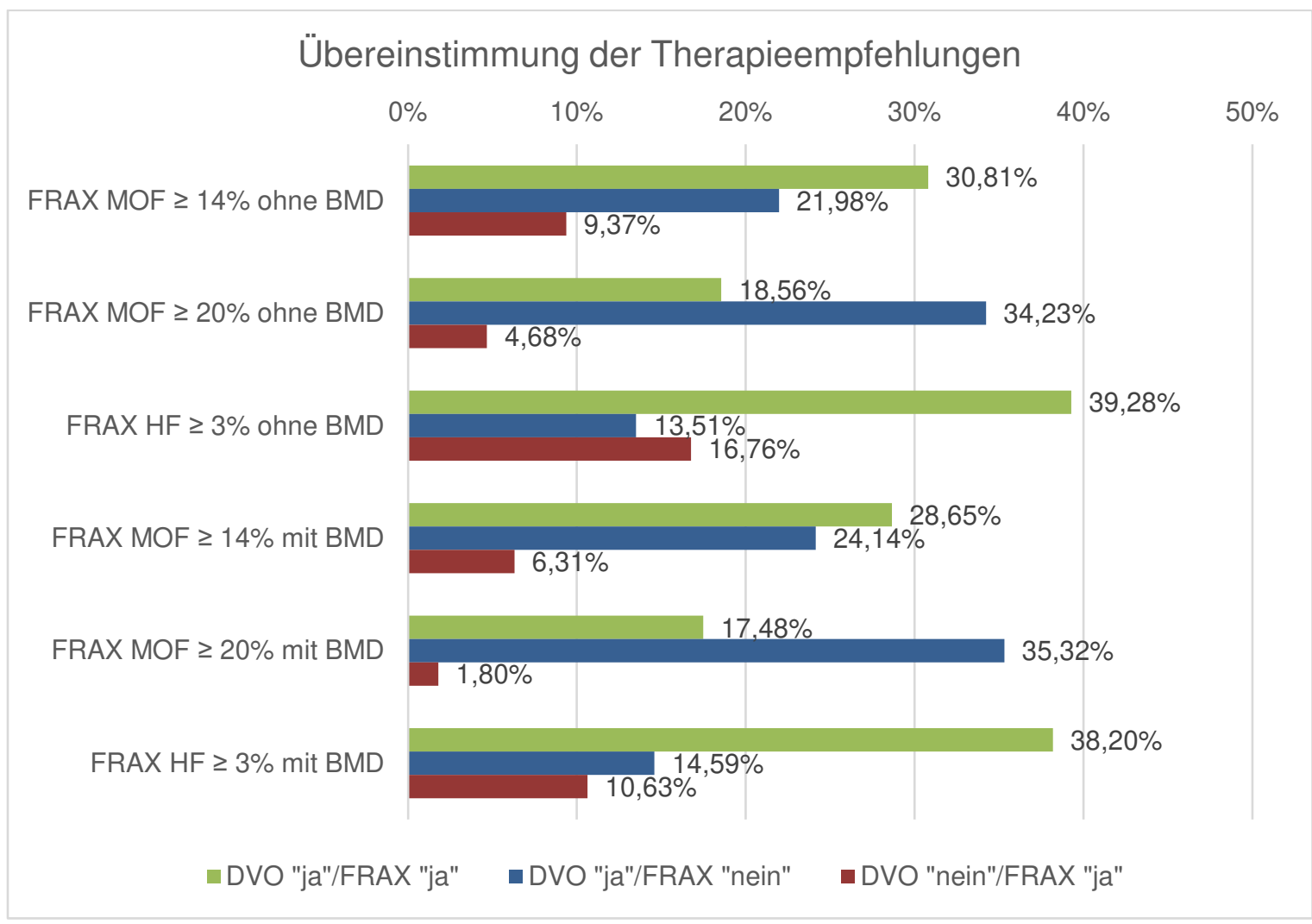

Abbildung 28: Patientinnenbezogene Übereinstimmung der Therapieempfehlungen ( $n=555)$. Grün: Therapieempfehlung laut DVO und laut FRAX; Blau: Therapieempfehlung nur laut DVO; Rot: Therapieempfehlung nur laut FRAX.

\subsection{Regression FRAX}

Mittels Regressionsfunktion im Statistikprogramm STATA wurden die in den FRAX-Rechner eingehenden Risikofaktoren sowie die Knochendichte auf deren Einfluss auf das ausgegebene 10-Jahres-Frakturrisiko MOF bzw. HF untersucht.

\subsubsection{Regression FRAX MOF mit und ohne BMD}

Tabelle 24 zeigt, dass ohne einbezogene Knochendichtemessung alle anderen Faktoren hochsignifikanten Einfluss auf den produzierten FRAX-10Jahresrisikowert MOF hatten. Bei einbezogener Knochendichtemessung nahmen Gewicht und Größe keinen signifikanten Einfluss mehr. Ebenfalls war der Einfluss des Faktors "sekundäre Osteoporose ja/nein" nicht mehr signifikant. Der Faktor "gegenwärtiges Rauchen ja/nein" änderte sich von hochsignifikant zu signifikant einflussnehmend. Den höchsten Einfluss nahm laut Regression sowohl bei als 
auch bei nicht einfließender Knochendichtemessung der Faktor „Hüftfraktur eines Elternteils ja/nein“. Bei nicht eingehendem T-Score folgt der Faktor „vorausgegangene Fraktur ja/nein“. Der Koeffizient ändert sich allerdings bei eingehendem T-Score von 7,85 zu 5,47 .

Tabelle 24: Regression FRAX MOF mit und ohne BMD

\begin{tabular}{|c|c|c|c|c|c|c|}
\hline \multirow[b]{2}{*}{$\begin{array}{c}\text { eingehende Risiko- } \\
\text { faktoren }\end{array}$} & \multicolumn{3}{|c|}{ FRAX MOF ohne BMD } & \multicolumn{3}{|c|}{ FRAX MOF mit BMD } \\
\hline & $\begin{array}{l}\text { Koef- } \\
\text { fizient }\end{array}$ & $\begin{array}{c}\text { robuster } \\
\text { Standard- } \\
\text { fehler }\end{array}$ & $\begin{array}{c}\text { Signifikanz- } \\
\text { niveau } \\
\text { (p-Wert) }\end{array}$ & $\begin{array}{l}\text { Koef- } \\
\text { fizient }\end{array}$ & $\begin{array}{c}\text { robuster } \\
\text { Standard- } \\
\text { fehler }\end{array}$ & $\begin{array}{c}\text { Signifikanz- } \\
\text { niveau } \\
\text { (p-Wert) }\end{array}$ \\
\hline Alter (Jahre) & 0,67 & 0,03 & $0,00^{* *}$ & 0,3 & 0,02 & $0,00^{* *}$ \\
\hline Gewicht (kg) & $-0,15$ & 0,02 & $0,00^{* *}$ & 0,02 & 0,02 & 0,24 \\
\hline Größe (cm) & 0,08 & 0,03 & $0,00^{* *}$ & $-0,03$ & 0,03 & 0,33 \\
\hline $\begin{array}{c}\text { vorausgegangene } \\
\text { Fraktur }\end{array}$ & 7,85 & 0,32 & $0,00^{* *}$ & 5,47 & 0,35 & $0,00^{* *}$ \\
\hline $\begin{array}{c}\text { Femurfraktur eines } \\
\text { Elternteils }\end{array}$ & 8,4 & 0,83 & $0,00^{* *}$ & 8,31 & 0,97 & $0,00^{* *}$ \\
\hline $\begin{array}{c}\text { Gegenwärtiges Rau- } \\
\text { chen }\end{array}$ & 1,38 & 0,44 & $0,00^{* *}$ & 1,28 & 0,57 & $0,02^{*}$ \\
\hline Glukokortikoide & 6,65 & 0,51 & $0,00^{\star *}$ & 5,85 & 0,62 & $0,00^{\star *}$ \\
\hline $\begin{array}{c}\text { Rheumatoide Arthri- } \\
\text { tis }\end{array}$ & 6,06 & 1,1 & $0,00^{* *}$ & 6,41 & 1,44 & $0,00^{* *}$ \\
\hline $\begin{array}{c}\text { sekundäre Osteopo- } \\
\text { rose }\end{array}$ & 4,58 & 0,5 & $0,00^{* *}$ & 0,21 & 0,45 & 0,65 \\
\hline Alkoholkonsum & 2,71 & 0,55 & $0,00^{* *}$ & 2,37 & 0,62 & $0,00^{\star \star}$ \\
\hline T-Score & & & & $-4,7$ & 0,41 & $0,00^{* *}$ \\
\hline Gesamt & $-41,09$ & 4,85 & $0,00^{* *}$ & $-17,32$ & 5,91 & $0,00^{* *}$ \\
\hline
\end{tabular}

\subsubsection{Regression FRAX HF mit und ohne BMD}

Ebenfalls wurde eine Regression für FRAX HF mit und ohne BMD durchgeführt. Es ergab sich ein signifikanter Einfluss aller Faktoren außer dem Alkoholkonsum ohne eingehenden T-Score. Wie schon bei dem FRAX-Wert MOF änderte sich auch beim Risiko für eine HF die Einflussstärke von Gewicht, Größe und sekundärer Osteoporose zu nicht mehr signifikant bei eingehendem T-Score. Generell zeigte sich das Niveau der Koeffizienten niedriger als bei Betrachtung der MOF. Den höchsten Koeffizienten erhielt erneut der Faktor „Hüftfraktur eines Elternteils ja/nein“. Der Koeffizient für eine vorausgegangene Fraktur liegt mit 3,99 bzw. 2,22 auch im Verhältnis zu den anderen Risikofaktoren deutlich niedriger als bei der Regression für eine MOF. 
Tabelle 25: Regression FRAX HF mit und ohne BMD

\begin{tabular}{|c|c|c|c|c|c|c|}
\hline \multirow[b]{2}{*}{$\begin{array}{c}\text { eingehende Risiko- } \\
\text { faktoren }\end{array}$} & \multicolumn{3}{|c|}{ FRAX HF ohne BMD } & \multicolumn{3}{|c|}{ FRAX HF mit BMD } \\
\hline & $\begin{array}{l}\text { Koef- } \\
\text { fizient }\end{array}$ & $\begin{array}{c}\text { robuster } \\
\text { Standard- } \\
\text { fehler }\end{array}$ & $\begin{array}{c}\text { Signifikanz- } \\
\text { niveau } \\
\text { (p-Wert) }\end{array}$ & $\begin{array}{l}\text { Koef- } \\
\text { fizient }\end{array}$ & $\begin{array}{c}\text { robuster } \\
\text { Standard- } \\
\text { fehler }\end{array}$ & $\begin{array}{c}\text { Signifi- } \\
\text { kanzniveau } \\
\text { (p-Wert) }\end{array}$ \\
\hline Alter (Jahre) & 0,5 & 0,03 & $0,00^{\star *}$ & 0,18 & 0,03 & $0,00^{\star *}$ \\
\hline Gewicht (kg) & $-0,14$ & 0,02 & $0,00^{* *}$ & 0 & 0,02 & 0,87 \\
\hline Größe (cm) & 0,08 & 0,03 & $0,00^{* *}$ & $-0,01$ & 0,03 & 0,84 \\
\hline $\begin{array}{c}\text { vorausgegangene } \\
\text { Fraktur }\end{array}$ & 3,99 & 0,35 & $0,00^{* *}$ & 2,22 & 0,36 & $0,00^{* *}$ \\
\hline $\begin{array}{c}\text { Femurfraktur eines } \\
\text { Elternteils }\end{array}$ & 5,61 & 1,07 & $0,00^{* *}$ & 4,86 & 1,24 & $0,00^{* *}$ \\
\hline $\begin{array}{c}\text { Gegenwärtiges Rau- } \\
\text { chen }\end{array}$ & 1,47 & 0,41 & $0,00^{* *}$ & 1,93 & 0,57 & $0,00^{* *}$ \\
\hline Glukokortikoide & 3,99 & 0,52 & $0,00^{* *}$ & 2,78 & 0,64 & $0,00^{* *}$ \\
\hline $\begin{array}{c}\text { Rheumatoide Arthri- } \\
\text { tis }\end{array}$ & 5,42 & 1,28 & $0,00^{* *}$ & 4,9 & 1,75 & $0,01^{*}$ \\
\hline $\begin{array}{c}\text { sekundäre Osteopo- } \\
\text { rose }\end{array}$ & 2,79 & 0,56 & $0,00^{* *}$ & 0,02 & 0,45 & 0,97 \\
\hline Alkoholkonsum & 0,94 & 0,54 & 0,09 & 0,95 & 0,61 & 0,12 \\
\hline T-Score & & & & $-3,73$ & 0,43 & $0,00^{* *}$ \\
\hline Gesamt & $-34,15$ & 4,95 & $0,00^{\star *}$ & $-15,32$ & 6,21 & $0,01^{*}$ \\
\hline
\end{tabular}

\subsection{Vergleich FRAX und modifizierter FRAX „endo“}

Wie bereits im Methodenteil angeführt wurde nicht nur das Risiko laut FRAX sondern ergänzend dazu auch ein Frakturrisiko genannt FRAX „endo“ erzeugt. Bei letzterem flossen als sekundäre Osteoporoseursachen nicht die laut FRAXEingabeseite zu verwendenden Ursachen ein, sondern stattdessen die im MVZ endokrinologikum verwendete Liste an sekundären Osteoporoseursachen. Von den 555 Patientinnen hatten 126 Patientinnen eine sekundäre Osteoporose laut FRAX, 116 laut DVO-Score. Nur 33 dieser Patientinnen stimmten überein. Es ergab sich kein signifikanter Unterschied zwischen den Risikowerten von FRAX und FRAX „endo“ weder für MOF noch HF jeweils mit und ohne eingeschlossene Knochendichtemessung. 
Tabelle 26: Vergleich FRAX und FRAX "endo"

\begin{tabular}{l|ccccc} 
& Mittelwert & SD & Maximum & Minimum & Median \\
\hline FRAX MOF ohne BMD & 14,18 & 10,47 & 65,0 & 2,1 & 11,0 \\
FRAX ,endo“ MOF ohne BMD & 14,18 & 10,62 & 65,0 & 2,3 & 11,0 \\
\hline FRAX HF ohne BMD & 6,29 & 8,08 & 55,0 & 0,1 & 3,6 \\
FRAX ,endo“ HF ohne BMD & 6,36 & 8,17 & 55 & 0,1 & 3,5 \\
\hline FRAX MOF mit BMD & 13,24 & 9,81 & 86,0 & 2,0 & 11,0 \\
FRAX,,endo“ MOF mit BMD & 13,31 & 10,09 & 86,0 & 2,0 & 11,0 \\
\hline FRAX HF mit BMD & 5,39 & 7,65 & 84,0 & 0,0 & 2,8 \\
FRAX ,endo“ HF mit BMD & 5,38 & 7,65 & 84,0 & 0 & 2,7
\end{tabular}

Vergleich der deskriptiven Statistik von FRAX und FRAX „endo“ mit und ohne Knochendichtemessungsergebnis $(n=555)$

\subsubsection{Vergleich Therapieentscheidungen FRAX und FRAX „endo“}

Weiterhin wurde verglichen inwiefern sich FRAX und FRAX „endo“ hinsichtlich der Therapieentscheidungen bei zugrunde gelegten Therapiegrenzen von MOF $\geq 20 \%$ und $\mathrm{HF} \geq 3 \%$ Frakturrisiko unterschieden. Bei eingeschlossener Knochendichtemessung unterschieden sich FRAX und FRAX „endo" weder bei den Therapieempfehlungen mit zugrunde gelegter Therapiegrenze der MOF $\geq 20 \%$, noch der $\mathrm{HF} \geq 3 \%$ noch der zusammengenommenen Therapieschwelle untereinander. 


\section{Diskussion}

Verschiedene Scores zur Frakturrisikoevaluation bei Patientinnen mit Osteoporose stehen national und international zur Verfügung. Zur Prävention und rechtzeitigen Behandlung osteoporotischer Knochenveränderungen ist die Eruierung des individuellen Patientenrisikos essentiell. Während der FRAX-Risikorechner zwar an großen Patientenkollektiven international gut validiert ist, die erstellte Zielgröße aber keine Therapieschwelle beinhaltet, ist die Frakturrisikoeinschätzung des DVO weniger validiert, gibt allerdings klare Empfehlungen zu Risikogrenzen für Diagnostik und Therapie.

In der vorliegenden Arbeit wurden anerkannte (FRAX, DVO) und nichtpublizierte (DVO+) Frakturrisikoevaluationsscores untereinander hinsichtlich ihrer Risikoeinschätzung für das Auftreten osteoporotischer Frakturen und Therapieschwellen verglichen. Ergänzend wurden die Einflussstärken der einzelnen FRAXRisikofaktoren am vorliegenden Kollektiv bestimmt um die hinterlegte Matrix des FRAX näherungsweise zu entschlüsseln.

\subsection{Basisdaten innerhalb des Frauenkollektivs}

Zu berücksichtigen ist bei Betrachtung der Ergebnisse, dass es sich beim betrachteten Kollektiv durch die Vorstellung im MVZ endokrinologikum Göttingen als ambulantes Schwerpunktzentrum des DVO um ein stark vorselektiertes „krankes“ Patientenklientel handelt. Die in die Studie aufgenommen Patientinnen waren bereits wegen des Verdachts, zur Abklärung oder des Vorliegens einer Osteoporose in das MVZ überwiesen worden.

Im Folgenden werden die Daten dieses Patientinnenkollektivs mit unterschiedlichen internationalen Kollektiven verglichen.

Die OPUS Study schloss Patientinnen über dem 55. Lebensjahr ein und wurde prospektiv über sechs Jahre durchgeführt, um das FRAXFrakturvorhersagemodell an europäischen postmenopausalen Frauen in einer normalen Population zu bewerten (Briot et al. 2013).

Weiterhin wird Bezug genommen auf die Patienten des Manitoba Bone Density Programs (MBDP). Es handelte sich bei diesem Kollektiv um $(n=34060)$ Patientinnen und Patienten über dem 50. Lebensjahr lebend in Manitoba, Kanada, die bisher keine antiosteoporotische Therapie erhielten und nach regelhafter DXA- 
Messung registriert wurden. Der Frauenanteil des Kollektivs lag bei $91 \%$ (Leslie et al. 2016).

Weiterhin zum Vergleich herangezogen wird das Kollektiv von Marques et al (2017). In dieser Studie wurden drei Patientenkohorten mit Patienten über dem 40. Lebensjahr und vollständig erhobenen FRAX-Risikofaktoren aus Portugal erfasst und prospektiv das Frakturauftreten betrachtet (Marques et al. 2017).

Tabelle 27: Vergleich der Basisdaten mit anderen Studien

\begin{tabular}{|c|c|c|c|c|}
\hline & $\begin{array}{l}\text { MVZ endo- } \\
\text { krinologikum } \\
\text { Goettingen }\end{array}$ & $\begin{array}{l}\text { Manitoba Bone } \\
\text { Density Program }\end{array}$ & OPUS Study & $\begin{array}{c}\text { Marques et } \\
\text { al. }\end{array}$ \\
\hline$N$ & 555 & 34060 & 1748 & 1943 \\
\hline Alter in Jahren $( \pm S D)$ & $64,21( \pm 10,3)$ & $66,6( \pm 9,8)$ & $66,1( \pm 6,8)$ & $57,7( \pm 9,9)$ \\
\hline$B M I$ in $k g / m^{2}( \pm S D)$ & $24,94( \pm 4,66)$ & - & $26,7( \pm 4,5)$ & - \\
\hline$\underset{\text { tur }}{\text { Vorausgegangene Frak- }}$ & $52,61 \%$ & $14,4 \%$ & $43,1 \%$ & $18,5 \%$ \\
\hline $\begin{array}{c}\text { Hüftfraktur eines Eltern- } \\
\text { teils }\end{array}$ & $13,87 \%$ & - & $10,5 \%$ & $8,4 \%$ \\
\hline Gegenwärtiges Rauchen & $16,40 \%$ & - & $13,8 \%$ & $13,8 \%$ \\
\hline Glukokortikoideinnahme & $16,76 \%$ & $5,3 \%$ & $2,7 \%$ & $7,4 \%$ \\
\hline Rheumatoide Arthritis & $7,03 \%$ & - & $6,4 \%$ & $5,7 \%$ \\
\hline Alkoholkonsum & $8,65 \%$ & - & $0,6 \%$ & $12,5 \%$ \\
\hline $\begin{array}{l}\text { T-Score Femurhals } \\
( \pm S D)\end{array}$ & $-1,86( \pm 0,98)$ & $-1,5( \pm 1,0)$ & $-0,42( \pm 1,05)$ & $-1,58( \pm 1,3)$ \\
\hline Sekundäre Osteoporose & $22,70 \%$ & - & $30,2 \%$ & $30,5 \%$ \\
\hline 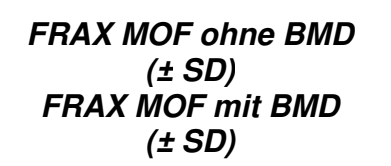 & $\begin{array}{c}14,18( \pm 10,47) \\
13,24( \pm 9,81)\end{array}$ & $12,3( \pm 8,1)$ & $\begin{array}{l}13,03( \pm 8,52) \\
10,23( \pm 7,02)\end{array}$ & - \\
\hline
\end{tabular}

Basisdaten und FRAX-Werte der Patientinnen des MVZ endokrinologikum Göttingen, des Manitoba Bone Density Program (Leslie et al. (2016)), der OPUS Study und des Kollektivs von Marques et al. (2017)

\subsubsection{Alter}

Das durchschnittliche Alter im betrachteten Kollektiv lag mit 64,21 $( \pm 10,3)$ Jahren knapp unter dem Durchschnittsalter der OPUS Study $(66,1 \pm 6,8)$, die allerdings auch nur Patientinnen über dem 55. Lebensjahr einschloss (Briot et al. 2013). Verglichen mit der BEST (66,6 Jahre) die männliche und weibliche OsteoporosePatienten über dem 50. Lebensjahr berücksichtige lag das Durchschnittsalter des vorliegenden Frauenkollektivs ebenfalls leicht niedriger (Hadji et al. 2013a). Das durchschnittliche Alter des MBDP lag mit 65,7 $( \pm 9,8)$ ebenfalls leicht über dem durchschnittlichen Alter dieser Studie. Eingeschlossen wurden hier ebenfalls nur Patienten über dem 50. Lebensjahr (Leslie et al. 2012). Das Alter des Kollektivs 
von Marques et al. (2017) lag mit 57,7 $( \pm 9,9)$ Jahren unter dem Alter dieses Kollektivs.

\subsubsection{Knochendichteparameter}

Bereits 1994 wurde durch die WHO-Definition die Diagnosestellung einer Osteoporose durch Vorliegen einer Knochendichte mit einem T-Score $\leq-2,5$ festgelegt. Studien zeigten, dass die Messung der Knochendichte am Femurhals eine bessere Vorhersage für das Risiko des Auftretens einer Hüftfraktur ermöglicht, als die alleinige Betrachtung der klinischen Risikofaktoren. Dargelegt wurde auch, dass die Aussagekraft der Knochendichtemessung am Femurhals für das Auftreten von Hüftfrakturen ein stärkerer Prädiktor ist, als die Messung an anderen Lokalisationen, einschließlich der Lendenwirbelsäule. Im Unterschied dazu ist die Risikoeinschätzung für andere Frakturlokalitäten als die Hüfte an allen anderen Messorten gleich valide (Kälvesten et al. 2016).

Im vorliegenden Kollektiv erfüllten bei Berücksichtigung des Messungsergebnisses am Femurhals 27,03 \% dieses Osteoporose-Kriterium der WHO. Der Mittelwert der T-Scores am Femurhals lag bei -1,86 ( \pm 0,98), verglichen mit dem Mittelwert der T-Scores der OPUS Study somit deutlich niedriger $(-0,42 \pm 1,05)$, zu erklären durch das vorselektionierte Patientenklientel unserer Studie. Auch die Werte für den T-Score der Wirbelsäule lagen in unserem Kollektiv niedriger als in dem der OPUS Study $(-0,95 \pm 1,53$ vgl. $-2,21 \pm 1,23)$ (Briot et al. 2013). Der T-Score am Femurhals der Patienten im MBDP lag bei $-1,5 \pm 1,0$. Der zusammengefasst minimale T-Score aller Lokalisationen eingeschlossen der Wirbelsäule lag hier bei $2,0 \pm 1,1$. Somit war er ebenfalls niedriger als der des alleinig betrachteten Femurhalses aber dennoch über dem des Kollektivs des MVZ endokrinologikum $(-2,51 \pm 0,99)$ (Leslie et al. 2016). Auch bei Marques et al. (2017) lag der durchschnittliche T-Score am Femurhals über dem des Kollektivs dieser Studie $(-1,58 \pm 1,3)$.

Die alleinige Verwendung eines Knochendichtemessungsergebnisses zur Frakturrisikoevaluation gilt mittlerweile allerdings als nicht ausreichend für eine multifaktoriell bedingte Erkrankung wie die Osteoporose. Eine Kombination von Knochendichtemessungsergebnis und Risikofaktoren für das Auftreten von osteoporotischen Knochenveränderungen und Frakturen zur Risikodetektion ist das Ergebnis dieser Überlegung. Eine Steigerung der Genauigkeit der Frakturprognose wird in 
Studien bereits diskutiert (Johansson et al. 2017). Die in dieser Arbeit untersuchten Scores haben diese Kombination auf unterschiedliche Weise umgesetzt.

\subsection{Score-Vergleich}

Wie eingangs erwähnt unterscheiden sich die verwendeten Risikoscores elementar hinsichtlich der Anzahl eingehenden Risikofaktoren sowie hinsichtlich des resultierenden Risikowertes bzw. der Risikowerte. Auch in der aktuellen Literatur wird länderübergreifend diskutiert, inwiefern komplexe Scores einen Vorteil gegenüber vereinfachten und damit in der klinischen Praxis vermeintlich besser zugänglichen Risikoevaluationstools haben. Rubin et al. (2013) zeigten, dass komplexere Risikomodelle nicht zu besseren Vorhersagequalitäten führten, als einfachere Risikomodelle.

Tabelle 28 stellt die Unterschiede von DVO-Leitlinie und FRAX vergleichend gegenüber.

Tabelle 28: Gegenüberstellung DVO und FRAX

\begin{tabular}{|c|c|}
\hline DVO & FRAX \\
\hline Zahlreiche Risikofaktoren & 12 Risikofaktoren \\
\hline Differenzierung der eingehenden Frakturtypen & „vorausgehende Fraktur“ \\
\hline $\begin{array}{l}\text { Minimale Knochendichte an LWS oder Femur } \\
\text { gesamt oder Femurhals }\end{array}$ & Minimale Knochendichte Femurhals (fakultativ) \\
\hline Mehrstufiges Vorgehen & Einstufiges Vorgehen mittels Eingabemaske \\
\hline Therapieschwelle einheitlich (> $30 \%$ ) & Keine einheitliche Therapieschwelle \\
\hline $\begin{array}{c}\text { Zielgröße: 10-Jahres-Frakturrisiko osteoporose- } \\
\text { assoziierte Frakturen }\end{array}$ & $\begin{array}{c}\text { Zielgröße: 10-Jahres-Frakturrisiko MOF oder } \\
\text { HF }\end{array}$ \\
\hline
\end{tabular}

Betrachtet man die Ergebnisse des international validierten und verwendeten FRAX-Scores und vergleicht diese mit den Risikoeinschätzungen der Patientinnen zugeordnet nach den Leitlinienempfehlungen des DVO fällt zuerst das unterschiedliche Niveau der Risikowerte auf. Im Schnitt liegen die FRAX-10-JahresRisikowerte für eine MOF bei eingeschlossener Knochendichtemessung bei dem hier vorgestellten Kollektiv bei 13,24 $( \pm 9,81)$. Im Vergleich zu den Werten der OPUS Study $(10,23 \pm 7,02)$ liegen die Werte des vorliegenden vorselektierten „Risikokollektivs“ wie zu erwarten etwas höher. Leslie et al. (2016) erhielten in einem ähnlichen Risikokollektiv beim Vergleich von FRAX mit einem Tool der Canadian Association of Radiologists and Osteoporosis Canada, genannt CAROC-Tool einen durchschnittlichen FRAX-Score für eine MOF von 12,3 $( \pm 8,1)$. 
Laut DVO werden erst Patientinnen mit geschätztem 10-Jahres-Frakturrisiko über $20 \%$ einer Basisdiagnostik zugeführt. Das unterschiedliche Risikoniveau der beiden Scores zeigt auch der Vergleich der übereinstimmenden Patientinnen sofern man den FRAX in die Risikogruppen $<20 \%, 20-30 \%$ sowie $>30 \%$ gruppiert. Insgesamt werden nur $11,9 \%$ der Patientinnen von DVO und FRAX MOF mit BMD übereinstimmend auf diese Gruppen verteilt. Sogar mit 13,3\% ein etwas größerer Patientenanteil wird von FRAX MOF ohne BMD und DVO übereinstimmend kategorisiert, obwohl der DVO ja ebenfalls ein Knochendichtemessungsergebnis einbezieht. Begründet liegen könnte die bessere tendenzielle Übereinstimmung bei nicht einbezogener Knochendichtemessung in den FRAX in der tendenziellen Korrektur des FRAX-Wertes mit BMD nach unten bei Patientinnen über dem 65. Lebensjahr (Vergleich Abbildung 14). Interessant sind in diesem Zusammenhang die Überlegungen von Kanis et al. (2016a): Die Knochendichte ändert bedingt durch die generelle pathophysiologische Abnahme der Knochendichte im Alter und den zunehmenden Einfluss der Mortalität ihre Einflussstärke auf das Frakturrisiko. Ein T-Score beispielsweise von $<-2,5$ kann im Alter von 80 Jahren paradoxerweise einen protektiven Einfluss darstellen.

Interessanterweise konnte die prospektive Studie von Marques et al. (2017). darlegen, dass die Einbeziehung der Knochendichtemessung in der FRAXRisikoberechnung in einer Normalpopulation keinen Benefit hinsichtlich der Frakturvorhersagegenauigkeit bringen konnte. In dem der Arbeit zugrundeliegenden Kollektiv lagen die durchschnittlichen FRAX-Risikowerte für eine MOF bzw. eine HF ohne BMD jeweils etwa $1 \%$ höher als die bei eingeschlossener Knochendichtemessung. Die Werte unterschieden sich jeweils signifikant voneinander. Auch auf die spätere Überlegung hinsichtlich einer Therapieindikationsgrenze hat die Eingabe von Knochendichtemessungsergebnissen im vorliegenden Kollektiv teilweise signifikanten Einfluss (siehe Therapievergleich).

\subsubsection{Vergleich der Score-Ergebnisse mit neuem Risikomodell DVO+}

Im Gegensatz zu DVO und FRAX wird durch das DVO+-Modell ein 1-JahresFrakturrisikowert erstellt. Folglich sollte bei Hochrechnung auf zehn Jahre durch die fehlende Einrechnung der Mortalität das Risiko für eine 10-Jahres-Fraktur überschätzt werden. Es zeigt sich bei Verteilung über die Altersgruppen des DVO+ eine höhere Risikowerteinschätzung für eine 1-Jahres-Fraktur laut DVO+ als laut FRAX vor allem ab dem 55. Lebensjahr. Nichtsdestotrotz ergeben sich 
signifikante Zusammenhänge zwischen den Score-Risikoeinschätzungen, was unter Berücksichtigung des Einflusses von Alter und zumindest teilweise übereinstimmenden Risikofaktoren durchaus zu erwarten war. Die niedrigsten Korrelationen finden sich dabei zwischen den FRAX-Werten mit BMD und dem DVO+.

\subsection{Therapievergleich}

Nur 12,3\% der Patientinnen mit Therapieindikation laut DVO erhielten auch im FRAX mit BMD ein Risiko für eine MOF $>30 \%$. Der durchschnittliche FRAX-Wert MOF mit BMD bei den nach DVO therapierten Patientinnen liegt bei 17,4\%, der durchschnittliche Wert für eine Hüftfraktur mit BMD liegt bei 8,1\%. Diese deutlich niedriger liegenden durchschnittlichen Mittelwerte im FRAX sowie die geringe Übereinstimmung der laut FRAX und DVO gleich kategorisierten Patientinnen legen die Überlegung nahe, dass den FRAX-Werten für eine Therapieentscheidung eine andere Therapiegrenze zugrunde gelegt werden sollte, als die $30 \%$ Frakturrisikogrenze des DVO.

Tatsächlich gibt es in der internationalen und nationalen Literatur hierzu verschiedene Überlegungen. Während die aktuellen Leitlinien des DVO eine Grenze von $\geq 14 \%$ für eine MOF und $\geq 3 \%$ für eine HF als den DVO-Therapiegrenzen entsprechend vorschlagen, wird in der internationalen Literatur ein höheres Risiko für eine MOF bei $\geq 20 \%$ Therapieindikation empfohlen (DVO 2014; Kanis et al. 2016b).

Auf das vorliegende Kollektiv wurden alle drei FRAX-Therapiegrenzen angewendet und die Übereinstimmung mit dem DVO verglichen. Weiterhin wurde betrachtet, wie viele der Patientinnen bei Überschreitung der Grenze für MOF und/oder HF therapiert werden würden. Es zeigte sich kein Unterschied zwischen den zusammengefassten Therapiegrenzen bei Überschreitung des jeweiligen Risikos für eine MOF ( $\geq 14 \%$ oder $\geq 20 \%$ ) und/oder HF ( $\geq 3 \%$ ) und den Therapiegrenzen sofern man nur die $\mathrm{HF} \geq 3 \%$ anwenden würde. Die Therapieschwelle $\mathrm{HF} \geq 3 \%$ stellt die empfindlichste Therapieschwelle dar.

$52,8 \%$ des Kollektivs, entsprechend 293 Patientinnen, wurde laut DVO ein Frakturrisiko über $30 \%$ 10-Jahres-Frakturwahrscheinlichkeit zugeordnet. Bei Vergleich der übereinstimmenden Therapieempfehlungen, also der Patientinnen, die laut DVO und FRAX übereinstimmend als zu therapieren bzw. nicht zu therapieren eingeordnet werden würden, zeigte sich kein signifikanter Unterschied zwischen dem DVO und dem FRAX mit BMD bei national und international verwendeter 
Therapiegrenze von $\geq 3 \%$ HF Risiko für eine 10-Jahres-Fraktur. Signifikante Unterschiede zwischen DVO und FRAX mit BMD gab es bei der Therapieentscheidung zugrunde gelegten Grenzen für eine 10-Jahres-Fraktur MOF. Sowohl bei $\geq 14 \%$ als auch bei $\geq 20 \%$ unterschieden sich die Empfehlungen signifikant von denen des DVO. Bei Bezug der therapierten Patientinnen laut FRAXEmpfehlungen auf den Anteil des Kollektivs mit Therapieindikation laut DVO ergab sich ein Anteil von jeweils knapp über $70 \%$ an Patientinnen, die bei Therapieindikation laut DVO ebenfalls im FRAX mit sowie ohne BMD bei Therapiegrenze $\geq 3 \% \mathrm{HF}$ therapiert werden würden.

Insgesamt wurde betrachtet wie hoch die mittleren FRAX-Werte bei den laut DVO therapierten Patientinnen waren. Der FRAX-Mittelwert für eine Hüftfraktur mit BMD lag bei 8,1 $( \pm 9,1)$ und damit deutlich über der Therapiegrenze von $\mathrm{HF} \geq 3 \%$. Es wurde daher auch der Mittelwert in der Gruppe der Patientinnen laut DVO 20-30 \% 10-Jahres-Frakturwahrscheinlichkeit betrachtet. Hier lag das mittlere Risiko für eine HF mit BMD bei 2,6\% $( \pm 3,9)$. Ohne einbezogene Knochendichtemessung jedoch lag der durchschnittliche Wert für eine HF bei 3,4 \% $( \pm 4,7)$ und damit über der Therapieschwelle laut FRAX. Mehr Patientinnen würden bei Therapieschwelle $\mathrm{HF} \geq 3 \%$ ohne BMD therapiert werden als laut DVO. Tatsächlich zeigt sich auch bei einem direkten Auszählen der Therapieempfehlungsübereinstimmung aller 555 Patientinnen, dass bei Therapiegrenze HF $\geq 3 \%$ ohne BMD $39,28 \%$ übereinstimmend laut DVO und FRAX als therapiewürdig erkannt werden, 16,76 \% jedoch nicht im DVO schon aber laut FRAX. 13,51\% würden laut DVO, nicht jedoch laut FRAX therapiert werden. Somit ist in dieser Gruppe der Anteil der laut FRAX nicht aber laut DVO therapierten Patientinnen höher als umgekehrt. In der Gruppe der 293 Patientinnen mit Therapieindikation laut DVO lag außerdem der Mittelwert für eine MOF ohne BMD bei 18,2 $( \pm 11,4)$ und damit ebenfalls über der nationalen FRAX-Therapieschwelle MOF $\geq 14 \%$ (mit BMD 17,4 ( $\pm 10,8)$ ). In der Gruppe der 240 Patientinnen mit 20-30\% Risiko lag die Werte analog bei 9,8 $( \pm 7,1)$ ohne bzw. 8,9 $( \pm 5,6)$ mit BMD. Um die gleichen Patientinnen zu therapieren müsste folglich die Grenze etwa bei MOF $\geq 17 \%$ mit BMD bzw. HF $\geq 8 \%$ mit BMD angelegt werden.

Weiterhin wurde betrachtet inwiefern der Einfluss der Knochendichtemessungseingabe in den FRAX die Therapieindikationen verändert, da in der aktuellen Literatur der fehlende Einfluss der Knochendichtemessungsergebniseingabe in den 
FRAX-Rechner im Bezug auf die Frakturvorhersagegenauigkeit diskutiert wird (Marques et al. 2017). Es ergaben sich signifikante Unterschiede hinsichtlich der Therapieentscheidungen mit bzw. ohne BMD bei Therapieschwelle eines HF Risikos $\geq 3 \%$ sowie eines MOF Risikos $\geq 14 \%$. Auch insgesamt zeigte sich ein signifikanter Unterschied zwischen den Therapieempfehlungsübereinstimmungen mit und ohne BMD. Ein Anteil von 7,21\% des Gesamtkollektivs würde ohne einflieBende Knochendichtemessung mehr therapiert werden, als bei einfließender Knochendichtemessung (Therapiegrenze HF $\geq 3 \%$ )

Hinsichtlich der Richtigkeit der jeweiligen Einschätzungen und Empfehlungen von DVO sowie FRAX mit und ohne BMD kann anhand der untersuchten Daten keine Aussage getroffen werden. Allerdings wurde in der prospektiven Studie von Marques et al. (2017) gezeigt, dass innerhalb eines Normalkollektivs an Frauen das Risiko für eine MOF vom FRAX eher unterschätzt, dahingegen das Risiko für eine HF tendenziell überschätzt wird. Unter Berücksichtigung des Wissens, dass im dieser Arbeit zugrunde liegenden Kollektiv die gestellten Therapieindikationen nach FRAX fast ausschließlich durch das Risiko für eine $\mathrm{HF} \geq 3 \%$ bestimmt werden, sollte kritisch hinterfragt werden, inwiefern mit dieser Indikationsgrenze ggf. sogar zu viele Patientinnen therapiert werden würden.

Auch Hadji et al. (2013b) diskutieren nach Auswertung der FREEDOM Study die Erforderlichkeit der Anpassung des Schwellenwertes der DVO-Therapieindikation. Es hatte sich unter Therapie mit Denusomab in der Studie eine Risikoreduktion für neue vertebrale Frakturen auch bei nicht therapiebedürttigen Patientinnen ergeben und legt die Diskussion einer niedrigeren Therapieschwelle somit nahe.

Auch Leslie et al. (2016) registrierten innerhalb eines Patientenkollektivs für das MBDP Patientinnen mit einem FRAX-10-Jahres-Frakturrisiko > $20 \%$ für eine MOF ( $\mathrm{NICHT} \geq 20 \%$ ). Laut kanadischer Leitlinie wird das Frakturrisiko äquivalent zum Risiko beispielweise für kardiovaskuläre Ereignisse in niedriges (<20\%), moderates (10-20\%) und hohes (> $20 \%$ ) Frakturrisiko eingeteilt. Therapieindikation haben demnach alle Patientinnen mit einem hohen Frakturrisiko über zwanzig Prozent. 14,8\% der Patientinnen des MBDP Kollektivs erhielten ein über zwanzigprozentiges Risiko für eine MOF (Vergleich Patientinnen dieser Studie 22,52\% $(\geq 20 \%)$ ).

Die Guidelines der National Osteoporosis Foundation (NOF) empfehlen eine antiosteoporotische Therapie bei stattgehabter Hüft- oder Wirbelkörperfraktur, einem 
T-Score $<-2,5$ oder einem FRAX-Risikowert MOF $\geq 20 \%$ bzw. HF $\geq 3 \%$ für Patientinnen mit einem T-Score zwischen -1 und -2,5. Goldshtein et al. (2017) untersuchten ein Kollektiv von Patientinnen im Alter zwischen 50 und 90 Jahren in Israel um die Güte verschiedener Therapiegrenzen zu bewerten. 30,5\% der Patientinnen erfüllten die Therapiekriterien laut NOF, verglichen mit dem Patientenkollektiv dieser Arbeit bei bloßer Anwendung von $\geq 20 \mathrm{MOF} / \geq 3 \% \mathrm{HF}$ ein niedrigerer Anteil an Patientinnen (49,01\%), bei jedoch weniger vorselektioniertem Patientinnenklientel. Goldshtein et al. (2017) ermittelten an ihrem Kollektiv weiterhin die hohe Güte der Frakturvorhersage bei Anwendung der NOF-Empfehlungen verglichen mit der bloßen Therapiegrenzenfestlegung durch das Ergebnis der Knochendichtemessung.

\subsubsection{Alternatives Risikomodell DVO+}

Der DVO+ schlägt Therapiegrenzen bei einem 1-Jahres-Frakturrisiko $>6 \%$ bzw. $>3 \%$ bei T-Score < -2 vor. Es ergibt sich, dass am wenigsten Patientinnen mit dieser Therapieschwelle therapiert werden würden. Mit 23,34 \% würden nur knapp unter der Hälfte der Patientinnen Therapieindikation erhalten, die laut DVO bzw. FRAX mit BMD $\geq 3 \%$-HF-Risiko-Therapiegrenze therapiert werden würden. Die Anzahl der therapierten Patientinnen laut DVO+ liegt damit noch unter der Patientenanzahl, die bei Zugrundelegung einer Therapiegrenze für eine MOF $\geq 20 \%$ therapiert werden würde $(33,1 \%)$.

Verglichen mit dem anfangs genannten prozentualen Anteil des Kollektivs der laut WHO-Definition unter einer Osteoporose leidet, würden mit Therapiegrenzen des DVO+ sogar weniger Patientinnen therapiert werden, als unter diese Definition fallen $(27,03 \%$ vs. $23,24 \%)$.

Im Gegensatz zu den vorher betrachteten Scores gibt der alternative Score DVO+ keine 10-Jahres-Frakturwahrscheinlichkeit an, sondern erstellt ein 1-JahresFrakturrisiko. Hochgerechnet auf zehn Jahre wird folglich die Mortalität nicht mit einbezogen und das Risiko daher eigentlich tendenziell höher eingeordnet als in den verglichenen Scores.

Der DVO+ unterscheidet sich im Hinblick auf die Entscheidungen für eine Therapie signifikant von DVO sowie FRAX mit und ohne BMD.

Der Vergleich der Scores hinsichtlich der generellen Therapieempfehlungen im Gegensatz zur Betrachtung der bloßen Risikoeinschätzungen erscheint vor allem zusätzlich als sinnvoll, weil die Therapieempfehlungen der Scores an unterschied- 
liche Frakturrisikoniveaus angelehnt werden. Es resultieren somit unterschiedliche Übereinstimmungswerte zwischen den Scores untereinander und den Therapieindikationen untereinander.

\subsection{Regressionsanalyse FRAX}

Mithilfe einer linearen Regression sollte beispielhaft am Kollektiv der Patientinnen dieser Arbeit die Matrix, die hinter der FRAX-Eingabemaske steckt entschlüsselt werden, um bewerten zu können, wie sich die einzelnen Faktoren auf die ZielgröBe des FRAX auswirken. Alle in den FRAX-Rechner einzugebenden Risikofaktoren fließen bei Nichteinbezug der Knochendichte in die FRAX-Zielgröße für eine MOF ein. Für die Zielgröße Hüftfraktur ohne einfließende Knochendichtemessung konnte der Einfluss des Alkoholkonsums nicht als signifikant nachgewiesen werden.

Bei einbezogener Knochendichtemessung ändert sich die Anzahl der hochsignifikanten einflussnehmenden Risikofaktoren. Größe und Gewicht, sowie Vorliegen einer sekundären Osteoporose ,ja/nein“ sind für eine MOF sowie für eine HF nun zusätzlich nicht mehr einflussnehmend. Zu vermuten ist, dass der durch Größe und Gewicht ermittelte BMI zurate gezogen wird, um zu ermitteln ob ein Untergewicht vorliegt, das direkten Einfluss auf die Knochendichte hätte. Sofern die Knochendichte einbezogen wird, ist der Risikofaktor Untergewicht mit verminderter Knochendichte durch diese zur Genüge abgebildet (De Laet et al. 2005).

Hohe Einflussfaktoren sind vor allem die vorausgegangene Fraktur sowie die Hüftfraktur eines Elternteils.

Studien belegen den hohen Einfluss einer vorausgegangenen Fraktur auf das Risiko des Auftretens einer weiteren osteoporotischen Fraktur zusätzlich zu dem, was an Frakturrisiko durch eine verminderte Knochendichte erklärt werden kann (Kanis et al. 2004). Die Ergebnisse unserer Regression sind konform zu diesen Überlegungen. Der Einfluss einer vorausgegangenen Fraktur wird bei einfließender Knochendichte geringer, bleibt jedoch hochsignifikant. Weiterhin ergab eine Meta-Analyse aus verschiedenen Studien zur Frakturrisikoevaluation, dass eine vorausgegangene Hüftfraktur eines Elternteils das Risiko für eine osteoporotische Fraktur unabhängig von der Knochendichte signifikant erhöht (Kanis et al. 2004). Auch diese dem FRAX-Rechner zugrunde gelegte Überlegung wird durch die lineare Regression unterstützt: Der Einfluss des Vorliegens einer Hüftfraktur eines Elternteils bleibt auch bei einbezogener Knochendichte hochsignifikant und zeigt 
sich nur geringfügig niedriger als bei nicht einbezogenem Knochendichtemessungsergebnis.

Interessant ist der fehlende Einfluss des Vorliegens einer sekundären Osteoporose bei eingehender Knochendichtemessung. Es ergibt sich als Schlussfolgerung, dass in unserem Kollektiv der Einfluss des Knochendichtemessungsergebnisses den Faktor des Vorliegens einer sekundären Osteoporose aufhebt.

Die Einflussstärken der Faktoren Rauchen, Rheumatoide Arthritis, Glukokortikoideinnahme sowie Alkoholkonsum ändern sich für eine MOF mit oder ohne einbezogene Knochendichte nur geringfügig und scheinen somit unabhängig von der Knochendichte zu sein.

\subsection{Vergleich FRAX und FRAX ,endo“}

Bei Hinzuziehen anderer als der laut FRAX vorgesehenen Ursachen für eine sekundäre Osteoporose ergibt sich kein signifikanter Unterschied hinsichtlich der durchschnittlichen Risikowerte. Als sekundäre Ursache für eine Osteoporose laut FRAX gilt das Vorliegen eines der folgenden Faktoren: Typ I (insulinabhängiger) Diabetes, Osteogenesis imperfecta bei Erwachsenen, langjährige, unbehandelte Hyperthyreose, Hypogonadismus oder frühe Menopause (<45-jährig), chronische Mangelernährung oder Malabsorption und chronische Lebererkrankungen.

Die Verschlüsselung sekundärer Osteoporosen erfolgte im Studienzeitraum im MVZ endokrinologikum Göttingen nach einer dem damaligen Stand der jeweiligen DVO-Leitlinien zugrunde gelegten Liste mit sekundären Ursachen, die sich laborchemisch oder anderweitig in den Aufnahmeuntersuchungen zeigten. Zu den als sekundäre Osteoporose gewerteten Faktoren gehörten dabei zusätzlich zu den ebenfalls im FRAX überschneidend als sekundär berücksichtigten Faktoren der chronischen Lebererkrankungen, Hypogonadismus, Osteogenesis imperfecta und der Hyperthyreose sowie den in den FRAX extra eingehenden Faktoren Rheumatoide Arthritis sowie relevante Glukokortikoideinnahme und Alkoholkonsum, ein primärer oder sekundärer Hyperparathyreoidismus, genetische Ursachen, gastrointestinale Erkrankungen wie beispielsweise Morbus Crohn, Endokrinopathien, Hyper- oder Hypokalzurie, Antiepileptika-, Heparin-, Marcumar- oder Thyroxineinnahme, Z.n. Transplantation, Schwangerschaft, Metastasen, Vitamin-DMangel, das Vorliegen einer lokalisierten Osteoporose und die Mastozytose. Auch bei Eingabe nicht nur der laut FRAX vorgegebenen sekundären Osteoporosen, sondern bei Berücksichtigung des breiteren Katalogs an sekundären Osteo- 
porosen des MVZ endokrinologikum Göttingen (FRAX „endo“) ergibt sich kein signifikanter Unterschied zwischen den Risikowerten im Generellen oder den Therapiegrenzenüberschreitungen im Speziellen.

Einschränkend muss dafür allerdings berücksichtigt werden, dass der laut MVZ Katalog und somit im FRAX „endo“ nicht als sekundär berücksichtigte Faktor der frühzeitigen Menopause für die FRAX sekundären Osteoporosen von den 22,7 \% mit sekundärer Osteoporose ,ja“ ein Anteil von 95,2 \% ausmachte. Insgesamt erhielten laut FRAX sogar mehr Patientinnen, nämlich 126, den Risikofaktor „sekundäre Osteoporose ja“, als mittels FRAX „endo“ mit dem es nur 116 Patientinnen waren. Von diesen Patientinnen wurden nur 33 überschneidend nach beiden Listen als sekundäre Osteoporose ,ja“ verschlüsselt. Nichtsdestotrotz zeigt der vorgenommene Vergleich, dass auch die Registrierung anderer sekundärer Ursachen die Risikowerte insgesamt nicht ausschlaggebend verändert. Bestätigt wird dieses Ergebnis durch das Ergebnis der Regressionsanalyse. Sobald ein Knochendichtemessungsergebnis in den FRAX-Rechner eingegeben wurde, erwies sich laut dieser der Faktor „sekundäre Osteoporose ja/nein“ nicht mehr als signifikant einfließend. 


\subsection{Kritische Anmerkungen zur Studie}

Durch die Vorselektion der Patientenklientel durch die generelle Registrierung von Patientinnen im MVZ endokrinologikum Göttingen als Schwerpunktzentrum und die Akquise von Patientinnen zum Einschluss in die Studie, die bereits mit einer Osteoporose oder zur Abklärung einer Osteoporose im MVZ verschlüsselt wurden, handelt es sich nicht um ein der Normalbevölkerung entsprechendes, also epidemiologisches Kollektiv, sondern gegenteilig um ein vorselektiertes „krankes“ Patientenkollektiv.

Nicht berücksichtigt wurde weiterhin in der Auswertung, ob Patientinnen bei erstmaliger Vorstellung schon eine spezifische antiosteoporotische Therapie erhielten. Es wurde das generelle 10-Jahres-Frakturrisiko berechnet. In den Risikoscores gibt es nicht die Möglichkeit eine Risikoreduktion durch vorausgegangene Therapieeinleitung zu berücksichtigen. Die Reduzierung des Risikos durch eine eventuelle medikamentöse Therapie konnte daher nicht mit einbezogen werden, obgleich die aktuelle Literatur von einer Risikoreduktion durch antiosteoporotische Therapie über $50 \%$ ausgeht (Aggarwal und Masuda 2018).

Eine Aussage zur Güte der einzelnen Scores ist aus der Auswertung dieser Arbeit nicht abzuleiten und war nicht Ziel der Arbeit. Eine Validierung der Risikoeinschätzung des DVO an großen Kollektiven ist bisher nicht erfolgt - eine prospektive Betrachtung der in dieser Studie eingeschlossenen Patientinnen und Registrierung der stattgehabten Frakturen kann weiteren Aufschluss bringen.

Weiterhin ist kritisch anzumerken, dass beim Vergleich des FRAX mit dem modifizierten FRAX „endo“ die sekundären Ursachen des MVZ endokrinologikum Göttingen nicht als zusätzlich zu den Risikofaktoren des FRAX interpretiert werden dürfen, da für den FRAX „endo“ die frühzeitige Menopause nicht als sekundärer Risikofaktor einbezogen wurde.

\subsection{Ausblick}

Anhand der vorliegenden Daten kann keine Aussage getroffen werden, mit welchem der erwähnten Scores die „richtige“ Frakturrisikoeinschätzung erfolgte. Während der FRAX international gut validiert ist, fehlt die Validierung der DVOLeitlinien an großen Kollektiven im Rahmen von prospektiven Studien mit Registrierung der tatsächlich aufgetretenen Frakturen. 
Eine Analyse, welche der Patientinnen in den Jahren nach erstmaliger Vorstellung im MVZ endokrinologikum Göttingen der Einleitung einer Therapie zustimmten, bzw. wie viele und welche der Patientinnen im Folgenden Frakturen erlitten, gäbe die Möglichkeit am Kollektiv der Studie die Aussagekraft der verglichenen Scores zu beurteilen. 


\section{Zusammenfassung}

Bei der dieser Dissertation zugrundeliegenden Studie handelt es sich um eine retrospektive Analyse der Patientendaten von 555 weiblichen Patientinnen, die im Studienzeitraum zwischen Juli 2007 und Juni 2014 im MVZ endokrinologikum Göttingen wegen Osteoporose vorstellig wurden.

Untersucht wurde, inwieweit sich der international verwendete FRAX-Score von den Empfehlungen und Risikostratifizierungen der Leitlinien des deutschen Dachverbandes Osteologie e.V. (DVO) unterscheidet. Dabei wurden auch die Unterschiede bei Einbezug bzw. Nichteinbezug eines Knochendichtemessungsergebnisses in den FRAX-Rechner betrachtet. Zusätzlich dazu erfolgte der Vergleich mit einem neuen, nichtpublizierten Risikoscore, genannt DVO+, der auf Grundlage der DVO-Leitlinien von 2014 testweise von C. Glüer, Universität Kiel, erstellt wurde und eine 1-Jahres-Frakturwahrscheinlichkeit unter Einbeziehung eines umfangreichen Sets von Risikofaktoren zusätzlich zu Alter und Knochendichtemessungsergebnis ausgibt (Glüer 2014).

Der Vergleich der genannten Scores erfolgte nicht nur auf Grundlage der durchschnittlichen Frakturrisiken, sondern auch hinsichtlich der daraus abgeleiteten Therapieschwellen und der resultierenden Übereinstimmungen und Unterschiede hinsichtlich der Therapieindikationsstellung im Frauenkollektiv. Da der FRAXScore an sich keine Empfehlungen zu einer Therapieindikationsschwelle ausgibt, wurden die Therapieempfehlungen des DVO mit Empfehlungen zu Therapiegrenzen im FRAX - ausgesprochen von den DVO-Leitlinien 2014 - sowie mit international verwendeten Therapiegrenzen verglichen und es wurde eruiert, wo beim vorliegenden Kollektiv die Therapiegrenze laut FRAX liegen müsste, um die gleichen Patientinnen wie laut DVO zu therapieren.

Da der Benutzer die dem FRAX-Rechner zugrundeliegende Matrix bei Eingabe nicht nachvollziehen kann, wurde anhand des betrachteten Kollektivs weiterhin eine lineare Regression durchgeführt, um die Matrix näherungsweise zu entschlüsseln und den Einfluss der einzelnen Risikofaktoren im FRAX zu eruieren.

Zuletzt erfolgte der Vergleich vom regelhaft erzeugten FRAX-Risikowert mit einem alternativ erzeugten FRAX-Wert, genannt FRAX „endo“, für dessen Erstellung in den FRAX-Rechner nicht die laut FRAX einbezogenen sekundären Osteoporo- 
seursachen eingingen, sondern alle Ursachen, die im Studienzeitraum laut MVZ endokrinologikum Göttingen als sekundär verschlüsselt wurden.

Es ergab sich im untersuchten Kollektiv, dass der FRAX-Mittelwert für ein 10-Jahres-Frakturrisiko der Patientinnen, die laut DVO ein $>30 \%$ 10-JahresFrakturrisiko hatten, wesentlich niedriger lag als $30 \%$ (MOF 18,2\% ohne BMD und MOF 17,4\% mit BMD). Da laut aktueller Literatur eine Therapiegrenze von MOF $\geq 14 \%$ bzw. $\geq 20 \%$ sowie HF $\geq 3 \%$ empfohlen wird, wurde betrachtet, inwiefern die Übereinstimmung der Frakturwahrscheinlichkeiten zwar differiert, die Therapieindikationen jedoch übereinstimmten. Tatsächlich zeigte sich kein signifikanter Unterschied in den allgemeinen Therapieempfehlungen (Patientinnen übereinstimmend ,ja“ bzw. übereinstimmend „nein“) zwischen DVO und FRAX mit und ohne BMD bei den zugrundegelegten Therapieschwellen. Aufgeschlüsselt ergab sich, dass der fehlende signifikante Unterschied durch die hohe Übereinstimmung zwischen den laut DVO therapierten und den laut FRAX HF $\geq 3 \%$ therapierten Patientinnen entstand. Die Empfehlungen zwischen DVO und MOF $\geq 14 \%$ bzw. MOF $\geq 20 \%$ hingegen unterschieden sich signifikant.

Die durchgeführte lineare Regression zeigte die unterschiedlichen Einflussstärken der in den FRAX eingehenden Risikofaktoren. Es erwiesen sich alle berücksichtigten Faktoren als signifikant Einfluss nehmend auf das Risiko einer MOF, sofern kein Knochendichtemessungsergebnis einberechnet wurde. Bei einberechnetem Knochendichtemessungsergebnis änderten sich die Einflussstärke der Faktoren. Weiterhin erwiesen sich Größe und Gewicht sowie der Faktor sekundäre Osteoporose ,ja/nein" als nicht mehr signifikant Einfluss nehmend.

Der weiterhin bezüglich der sekundären Osteoporoseursachen durchgeführte Vergleich von FRAX und FRAX „endo“ ergab keinen signifikanten Unterschied zwischen den Werten des FRAX und den alternativen Werten des FRAX „endo“, weder hinsichtlich der generellen Frakturrisikomittelwerte noch hinsichtlich der daraus resultierenden Therapieempfehlungen.

Zukünftig sollte innerhalb prospektiver Studien die Frakturinzidenz im betrachteten Kollektiv eruiert werden, um die Validität der verschiedenen Scores in Deutschland zu evaluieren. 


\section{Literaturverzeichnis}

Aggarwal L, Masuda C (2018): Osteoporosis: A quick update. J Fam Pract $\underline{67}$, 5965

Ahmed LA, Shigdel R, Joakimsen RM, Eldevik OP, Eriksen EF, Ghasem-Zadeh A, Bala Y, Zebaze R, Seeman E, Bjornerem A (2015): Measurement of cortical porosity of the proximal femur improves identification of women with nonvertebral fragility fractures. Osteoporos Int $\underline{26}, 2137-2146$

Amling M (2015): Calcium and vitamin D in bone metabolism: Clinical importance for fracture treatment. Unfallchirurg $\underline{118}, 995-999$

Atteritano M, Catalano A, Santoro D, Lasco A, Benvenga S (2016): Effects of strontium ranelate on markers of cardiovascular risk in postmenopausal osteoporotic women. Endocrine $\underline{53}, 305-312$

Bartl R (Hrsg.): Osteoporose - Prävention, Diagnostik, Therapie. 4. Auflage; Georg Thieme Verlag, Stuttgart 2011

Baum E (2010): Die neue DVO-Leitlinie Osteoporose - ein Update. Arthritis Rheum 30, 7-12

Bernstein CN, Leslie WD, Leboff MS (2003): AGA technical review on osteoporosis in gastrointestinal diseases. Gastroenterology $\underline{124}, 795-841$

Bianchi G, Sambrook P (2008): Oral nitrogen-containing bisphosphonates: a systematic review of randomized clinical trials and vertebral fractures. Curr Med Res Opin 24, 2669-2677

Briot K, Paternotte S, Kolta S, Eastell R, Felsenberg D, Reid DM, Glüer CC, Roux C (2013): FRAX®: Prediction of major osteoporotic fractures in women from the general population: The OPUS study. PLoS ONE $\underline{\text { 8, e83436 }}$

Cameron ID, Dyer SM, Panagoda CE, Murray GR, Hill KD, Cumming RG, Kerse N (2018): Interventions for preventing falls in older people in care facilities and hospitals. Cochrane Database Syst Rev $\underline{9}$, CD005465

Chapuy MC, Arlot ME, Delmas PD, Meunier PJ (1994): Effect of calcium and cholecalciferol treatment for three years on hip fractures in elderly women. BMJ 308, 1081-1082

Chiodini I, Bolland MJ (2018): Calcium supplementation in osteoporosis: useful or harmful? Eur J Endocrinol 178, D13-25

Consensus Development Conference (1993): Diagnosis, prophylaxis and treatment of osteoporosis. Am J Med $\underline{94}, 646-650$ 
Cruz AS, Lins HC, Medeiros RVA, Filho JMF, da Silva SG (2018): Artificial intelligence on the identification of risk groups for osteoporosis, a general review. Biomed Eng Online 17, 12

DVO (2006): Prophylaxe, Diagnostik und Therapie der Osteoporose bei Frauen ab der Menopause, bei Männern ab dem 60. Lebensjahr. S3-Leitlinie des Dachverbands der Deutschsprachigen Wissenschaftlichen Osteologischen Gesellschaften e.V.

DVO (2009): DVO-Leitlinie 2009 zur Prophylaxe, Diagnostik und Therapie der Osteoporose im Erwachsenenalter, Kurzfassung. S3-Leitlinie des Dachverbands der Deutschsprachigen Wissenschaftlichen Osteologischen Gesellschaften e.V. https://www.gaertner-servatius.de/downloads/DVO-Leitlinie-2009-

Langfassung.pdf; abgerufen am 10.02.2020

DVO (2014): Prophylaxe, Diagnostik und Therapie der Osteoporose bei Männern ab dem 60. Lebensjahr und bei postmenopausalen Frauen. S3-Leitlinie des Dachverbands der Deutschsprachigen Wissenschaftlichen Osteologischen Gesellschaften e.V. http://www.dv-osteologie.org/uploads/Leitlinie\%202014/DVOLeitlinie\%200steoporose\%202014 \%20Kurzfassung\%20und\%20Langfassung\%20 Version\%201a\%2012 \%2001 \%202016.pdf; abgerufen am 10.02.2020

DVO (2017): Prophylaxe, Diagnostik und Therapie der Osteoporose bei Männern ab dem 60. Lebensjahr und bei postmenopausalen Frauen. S3-Leitlinie des Dachverbands der Deutschsprachigen Wissenschaftlichen Osteologischen Gesellschaften e.V. https://www.dv-osteologie.org/uploads/Leitlinie\%202017/Finale\%20Version\%20Leitlinie\%20Osteoporose\%202017_end.pdf; abgerufen am 10.02.2020

Erläuterungen zur DVO-Leitlinie (2009): Kommentierte Version der Langfassung der DVO-Leitlinie 2009

Faßbender WJ, Stumpf UC (2006): DVO-Leitlinie 2006. Z Rheumatol 65, 364-369

Faßbender WJ, Scheidt-Nave C, Pfeilschifter J (2003): Die neuen Leitlinien zur Osteoporose. Empfehlung des Dachverbandes der deutschsprachigen osteologischen Fachgesellschaften. Dtsch Med Wochenschr 128, 1615-1618

Feng X, McDonald JM (2011): Disorders of bone remodeling. Annu Rev Pathol $\underline{6}$, 121-145

Genant HK, Libanati C, Engelke K, Zanchetta JR, Hoiseth A, Yuen CK, Stonkus S, Bolognese MA, Franek E, Fuerst T, et al. (2013): Improvements in hip trabecular, subcortical, and cortical density and mass in postmenopausal women with osteoporosis treated with denosumab. Bone $\underline{56}, 482-488$

Geusens P, Marin F, Kendler DL, Russo LA, Zerbini CA, Minisola S, Body JJ, Lespessailles E, Greenspan SL, Bagur A, et al. (2018): Effects of Teriparatide Compared with Risedronate on the Risk of Fractures in Subgroups of Postmenopausal Women with Severe Osteoporosis: The VERO Trial. J Bone Miner Res $\underline{33}$, 783-794 
Glüer C (2014): Frakturrisiko für Hüft- und rad. WK Frakturen in Abhängigkeit von Alter sowie Anzahl klinischer Risikofaktoren. Präsentation im Rahmen des Osteologie-Kongress in München, 13.-15. März 2014

Goldshtein I, Ish-Shalom S, Leshno M (2017): Impact of FRAX-based osteoporosis intervention using real world data. Bone $\underline{103}, 318-324$

Hadji P, Klein S, Gothe H, Häussler B, Kless T, Schmidt T, Steinle T, Verheyen F, Linder R (2013a): The epidemiology of osteoporosis-Bone Evaluation Study (BEST): an analysis of routine health insurance data. Dtsch Arztebl Int 110, 52-57

Hadji P, Bock O, Resch H, Kraenzlin M, Wang A, Steinle T, Eisen C (2013b): Reduktion des Frakturrisikos unter Denusomab bei postmenopausalen Frauen in Abhängigkeit der Behandlungsbedürftigkeit nach DVO-Leitlinie 2009 Subgrppenanalyse der Freedom Study. Osteologie 22, 39-45

Haentjens P, Autier P, Collins J, Velkeniers B, Vanderschueren D, Boonen S (2003): Colles fracture, spine fracture, and subsequent risk of hip fracture in men

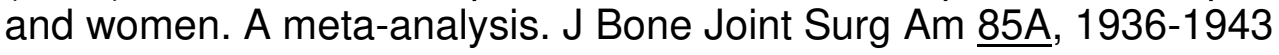

Hamilton CJ, Swan VJD, Jamal Sa (2010): The effects of exercise and physical activity participation on bone mass and geometry in postmenopausal women: a systematic review of PQCT studies. Osteoporos Int 21, 11-23

Häussler B, Gothe H, Mangiapane S, Glaeske G, Pientka L, Felsenberg D (2006): Versorgung von Osteoporose-Patienten in Deutschland Ergebnisse der BoneEVAStudie. Dtsch Arztebl 103, 2542-2548

Heaney RP, Abrams S, Dawson-Hughes B, Looker A, Marcus R, Matkovic V, Weaver C (2000): Peak Bone Mass. Osteoporos Int 11, 985-1009

Hernlund E, Svedbom A, Ivergard M, Compston J, Cooper C, Stenmark J, McCloskey EV, Jonsson B, Kanis JA (2013): Osteoporosis in the European Union: medical management, epidemiology and economic burden. A report prepared in collaboration with the International Osteoporosis Foundation (IOF) and the European Federation of Pharmaceutical Industry Associations (EFPIA). Arch Osteoporos $\underline{8}, 136$

Holick MF, Chen TC (2008): Vitamin D defciency: a worldwide problem with health consequences. Am J Clin Nutr $\underline{87}$, 1080S-1086S

Horne AM, Mihov B, Reid IR (2018): Bone Loss After Romosozumab/Denosumab: Effects of Bisphosphonates. Calcif Tissue Int 103, 55-61

Ismail A, Cockerill W, Cooper C, Finn J, Abendroth K, Parisi G, Banzer D, Benevolenskaya L, Bhalla A, Armas J, et al. (2001): Prevalent vertebral deformity predicts incident hip though not distal forearm fracture: results from the European Prospective Osteoporosis Study. Osteoporos Int 12, 85-90 
Johansson $\mathrm{H}$, Azizieh F, Al Ali N, Alessa T, Harvey NC, McCloskey E, Kanis JA (2017): FRAX- vs. T-Score-based intervention thresholds for osteoporosis. Osteoporos Int 28, 3099-3105

Kälvesten J, Lui L-Y, Brismar T, Cummings S (2016): Digital X-ray radiogrammetry in the study of osteoporotic fractures: Comparison to dual energy X-ray absorptiometry and FRAX. Bone $\underline{86}, 30-35$

Kanis JA on behalf of the World Health Organization Scientific Group: Assessment of osteoporosis at the primary health-care level. Technical Report. World Health Organization Collaborating Centre for Metabolic Bone Diseases, University of Sheffield, 2007

Kanis JA, Melton LJ, Christiansen C, Johnston CC, Khaltaev N (1994): The diagnosis of osteoporosis. J Bone Miner Res $\underline{9}, 1137-1141$

Kanis JA, Johnell O, Oden A, De Laet C, Jonsson B, Dawson A (2002): Ten-year risk of osteoporotic fracture and the effect of risk factors on screening strategies. Bone $\underline{30}, 251-258$

Kanis JA, Johnell O, De Laet C, Johansson H, Oden A, Delmas P, Eisman J, Fujiwara S, Garnero P, Kroger $\mathrm{H}$ et al. (2004): A meta-analysis of previous fracture and subsequent fracture risk. Bone $\underline{35}, 375-382$

Kanis JA, Oden A, Johnell O, Johansson H, De Laet C, Brown J, Burckhardt P, Cooper C, Christiansen C, Cummings S et al. (2007): The use of clinical risk factors enhances the performance of BMD in the prediction of hip and osteoporotic fractures in men and women. Osteoporos Int $\underline{18}$, 1033-1046

Kanis JA, McCloskey EV, Johansson H, Oden A, Ström O, Borgström F (2010): Development and use of FRAX® in osteoporosis. Osteoporos Int 21 1 , 407-413

Kanis JA, Harvey NC, McCloskey E (2014): Pre-screening young postmenopausal women for BMD testing. Bonekey Rep $\underline{3}, 544$

Kanis JA, Compston J, Cooper C, Harvey NC, Johansson H, Oden A, McCloskey EV (2016a): SIGN Guidelines for Scotland: BMD Versus FRAX Versus QFracture. Calcif Tissue Int $\underline{98}, 417-425$

Kanis JA, Harvey NC, Cooper C, Johansson H, Oden A, McCloskey EV (2016b): A systematic review of intervention thresholds based on FRAX: A report prepared for the National Osteoporosis Guideline Group and the International Osteoporosis Foundation. Arch Osteoporos 11, 25

Kurth AA, Pfeilschifter J (2007): Diagnostik und Therapie der postmenopausalen Osteoporose und der Osteoporose des Mannes: Update der Leitlinien 2006. Orthopade $\underline{36}$, 683-692

De Laet C, Kanis JA, Oden A, Johanson H, Johnell O, Delmas P, Eisman JA, Kroger H, Fujiwara S, Garnero P, et al. (2005): Body mass index as a predictor of fracture risk: a meta-analysis. Osteoporos Int 16, 1330-1338 
Leslie WD, Morin S, Lix LM, Johansson H, Oden A, McCloskey E, Kanis JA (2012): Fracture risk assessment without bone density measurement in routine clinical practice. Osteoporos Int $\underline{23}, 75-85$

Leslie WD, Majumdar SR, Lix LM, Josse RG, Johansson H, Oden A, McCloskey EV, Kanis JA (2016): Direct comparison of $\operatorname{FAX}(R)$ and a simplified fracture risk assessment tool in routine clinical practice: a registry-based cohort study. Osteoporos Int 27, 2689-2695

Lindsay R, Cooper C, Hanley Da, Barton I, Broy SB, Flowers K (2001): Risk of New Vertebral Fracture in the Year Following a Fracture. JAMA 285, 6-9

Lorentzon M, Cummings SR (2015): Osteoporosis: the evolution of a diagnosis. J Intern Med 277, 650-661

Marques A, Lucas R, Simoes E, Verstappen SMM, Jacobs JWG, da Silva JAP (2017): Do we need bone mineral density to estimate osteoporotic fracture risk? A 10 -year prospective multicentre validation study. RMD Open $\underline{3}$, e000509

Marx RE (2012): A Decade of Bisphosphonate Bone Complications: What It Has Taught Us About Bone Physiology. Oral Craniofac Tissue Eng 2, 309-320

McClung MR, Geusens P, Miller PD, Zippel H, Bensen WG, Roux C, Adami S, Fogelman I, Diamond T, Eastell R, et al. (2001): Effect of Risedronate on the risk of hip fracture in elderly women. $\mathrm{N}$ Engl J Med 344, 333-340

Mirza F, Canalis E (2015): Management of endocrine disease: Secondary osteoporosis: pathophysiology and management. Eur J Endocrinol 173, R131-151

Neuerburg C, Stumpf U, Schmidmaier R, Kammerlander C, Pfeilschifter J, Mutschler W, Böcker W (2015): Neue Osteoporose-Leitlinie DVO 2014 und ihre Bedeutung für den Unfallchirurgen. Unfallchirurg 118, 905-912

Niedhart C (2016): Osteoporosis: Diagnostics and Therapy According to the German Guidelines. Z Orthop Unfall 154, 237-244

Oral A, Kücükdeveci AA, Varela $E$, llieva $E M$, Valero $R$, Berteanu $M$, Christodoulou N (2013): Osteoporosis. The role of Physical and Rehabilitation Medicine Physicians. The European perspective based on the best evidence. Eur J Phys Rehabil Med 499, 565-577

Pfeilschifter J (2009): Osteoporose - Aktuelle Diagnostik und Therapie. Med Klin $\underline{104}, 632-643$

Pfeilschifter J (2010): Prophylaxe, Diagnostik und Therapie der Osteoporose bei Erwachsenen - Methodenreport - Verfahrensablauf zur Aktualisierung der DVOLeitlinie 2009 zur Prävention, Diagnostik und Therapie der primären und sekundären Osteoporose bei Erwachsenen. http://www.leitliniensekretariat.de/files/MyLayout/pdf/034-003m.pdf; abgerufen am 10.02.2020 
Reid IR, Bolland MJ, Grey A (2014): Effects of vitamin D supplements on bone mineral density: a systematic review and meta-analysis. Lancet $\underline{383}, 146-155$

Rossini M, Zanotti R, Orsolini G, Tripi G, Viapiana O, Idolazzi L, Zamò a, Bonadonna P, Kunnathully V, Adami S, et al. (2016): Prevalence, pathogenesis, and treatment options for mastocytosis-related osteoporosis. Osteoporos Int $\underline{27}$, 2411-2421

Roth A, Lehmann G, Wolf G (2013): Osteoporose - Diagnostik und Therapie. Z Ortho Unfall 2 , 122-128

Rubin KH, Friis-Holmberg T, Hermann AP, Abrahamsen B, Brixen K (2013): Risk assessment tools to identify women with increased risk of osteoporotic fracture: complexity or simplicity? A systematic review. J Bone Miner Res $\underline{28}, 1701-1717$

Saraff V, Hogler W (2015): Endocrinology and Adolesccence: Osteoporosis in children: diagnosis and management. Eur J Endocrinol $\underline{173}$, R185-197

Siggelkow H, Hadji P (2016): Osteoporose in der Gynäkologie - Diagnostik und Therapie. Frauenheilkd up2date 10, 203-218

Silverman SL, Chines AA, Kendler DL, Kung AWC, Teglbjærg CS, Felsenberg D, Mairon N, Constantine GD, Adachi JD (2012): Sustained efficancy and safety of bazedoxifene in preventing fractures in postmenopausal women with osteoporosis: Results of a 5-year, randomized, placebo-controlled study. Osteoporos Int $\underline{23}$, 351-363

Snellman G, Byberg L, Lemming EW, Melhus H, Gedeborg R, Mallmin H, Wolk A, Michaelsson K (2014): Long-term dietary vitamin D intake and risk of fracture and osteoporosis: a longitudinal cohort study of Swedish middle-aged and elderly women. J Clin Endocrinol Metab 99, 781-790

Steingrimsdottir L, Gunnarsson O, Indridason OS, Franzson L, Sigurdsson G (2005): Relationship Between Serum Parathyroid Hormone Levels, Vitamin D sufficiency and Calcium intake. JAMA 294, 2336-2341

Stewart TL, Ralston SH (2000): Role of genetic factors in the pathogenesis of osteoporosis. J Endocrinol 166, 235-245

Strom O, Borgstrom F, Kanis JA, Compston J, Cooper C, McCloskey EV, Jonsson B (2011): Osteoporosis: burden, health care provision and opportunities in the EU: a report prepared in collaboration with the International Osteoporosis Foundation (IOF) and the European Federation of Pharmaceutical Industry Associations (EFPIA). Arch Osteoporos $\underline{6}, 59-155$

Sutton EE, Riche DM (2012): Denosumab, a RANK ligand inhibitor, for postmenopausal women with osteoporosis. Ann Pharmacother 46, 1000-1009

Tella SH, Gallagher JC (2014): Prevention and treatment of postmenopausal osteoporosis. J Steroid Biochem Mol Biol 142, 155-170 
Tsai KS, Heath H, Kumar R, Riggs BL (1984): Impaired vitamin D metabolism with aging in women. Possible role in pathogenesis of senile osteoporosis. J Clin Invest $\underline{73}, 1668-1672$

Tu KN, Lie JD, Wan CKV, Cameron M, Austel AGJ, Nguyen JK, Van K, Hyun D (2018): Osteoporosis: A Review of treatment options. P T $\underline{43}, 92-104$

Winkelmann A, Schilling S, Neuerburg C, Mutschler W, Böcker W, Felsenberg D, Stumpf U (2015): Innovatives Bewegungstraining bei Osteoporose. Unfallchirurg $\underline{11}, 1-5$ 